\title{
Chern classes of automorphic vector bundles
}

\author{
Mark Goresky ${ }^{1}$ and William Pardon ${ }^{2}$
}

\section{INTRODUCTION}

1.1. Suppose $X$ is a compact $n$-dimensional complex manifold. Each partition $I=\left\{i_{1}, i_{2}\right.$, $\left.\ldots, i_{r}\right\}$ of $n$ corresponds to a Chern number $c^{I}(X)=\epsilon\left(c^{i_{1}}(X) \cup c^{i_{2}}(X) \cup \ldots \cup c^{i_{r}}(X) \cap[X]\right) \in \mathbb{Z}$ where $c^{k}(X) \in H^{2 k}(X ; \mathbb{Z})$ are the Chern classes of the tangent bundle, $[X] \in H_{2 n}(X ; \mathbb{Z})$ is the fundamental class, and $\epsilon: H_{0}(X ; \mathbb{Z}) \rightarrow \mathbb{Z}$ is the augmentation. Many invariants of $X$ (such as its complex cobordism class) may be expressed in terms of its Chern numbers ([Mi], [St]). During the last 25 years, characteristic classes of singular spaces have been defined in a variety of contexts: Whitney classes of Euler spaces $[\mathrm{Su}],[\mathrm{H}-\mathrm{T}],[\mathrm{Ak}]$, Todd classes of singular varieties $[\mathrm{BFM}]$, Chern classes of singular algebraic varieties [Mac], L-classes of stratified spaces with even codimension strata [GM1], Wu classes of singular spaces [Go2], [GP] (to name a few). However, these characteristic classes are invariably homology classes and as such, they cannot be multiplied with each other. In some cases it has been found possible to "lift" these classes from homology to intersection homology, where (some) characteristic numbers may be formed ([BBF], [BW], [Go2], [GP], [T]).

The case of locally symmetric spaces is particularly interesting. Suppose $\Gamma$ is a torsionfree arithmetic group acting on a complex $n$-dimensional Hermitian symmetric domain $D=G / K$, where $G$ is the group of real points of a semisimple algebraic group $\mathbf{G}$ defined over $\mathbb{Q}$ with $\Gamma \subset \mathbf{G}(\mathbb{Q})$, and where $K \subset G$ is a maximal compact subgroup. Then $X=\Gamma \backslash D$ is a Hermitian locally symmetric space. When $X$ is compact, Hirzebruch's proportionality theorem [Hr1] says that there is a number $v(\Gamma) \in \mathbb{Q}$ so that for every partition $I=\left\{i_{1}, i_{2}, \ldots, i_{r}\right\}$ of $n$, the Chern number satisfies $c^{I}(X)=v(\Gamma) c^{I}(\check{D})$, where $\check{D}=G_{u} / K$ is the compact dual symmetric space (and $G_{u}$ is a compact real form of $\mathbf{G}(\mathbb{C})$ containing $K$ ).

If $X=\Gamma \backslash D$ is noncompact, it has a canonical Baily-Borel (Satake) compactification, $\bar{X}$. This is a (usually highly singular) complex projective algebraic variety. To formulate a proportionality theorem in the noncompact case, one might hope that the tangent bundle $T X$ extends as a complex vector bundle over $\bar{X}$, but this is false. In [Mu1], D. Mumford showed that $T X$ has a particular extension $\bar{E}_{\Sigma} \rightarrow \bar{X}_{\Sigma}$ over any toroidal resolution $\tau: \bar{X}_{\Sigma} \rightarrow \bar{X}$ of the Baily-Borel compactification and that for any partition $I$ of $n$, the resulting Chern numbers

$$
c^{I}\left(\bar{E}_{\Sigma}\right)=\epsilon\left(c^{i_{1}}\left(\bar{E}_{\Sigma}\right) \cup c^{i_{2}}\left(\bar{E}_{\Sigma}\right) \cup \ldots \cup c^{i_{r}}\left(\bar{E}_{\Sigma}\right) \cap\left[\bar{X}_{\Sigma}\right]\right)
$$

\footnotetext{
${ }^{1}$ School of Mathematics, Institute for Advanced Study, Princeton N.J. Research partially supported by NSF grants \# DMS 9626616 and DMS 9900324.

${ }^{2}$ Dept. of Mathematics, Duke University, Durham N.C. Research partially supported by NSF grant \# DMS 9504900.
} 
satisfy the same equation, $c^{I}\left(\bar{E}_{\Sigma}\right)=v(\Gamma) c^{I}(\check{D})$. (The toroidal resolution $\bar{X}_{\Sigma}$ is constructed in $[\mathrm{AMRT}]$; it depends on a choice $\Sigma$ of polyhedral cone decompositions of certain selfadjoint homogeneous cones.) Mumford also showed that if $\Sigma^{\prime}$ is a refinement of $\Sigma$ then there is a natural morphism $f: \bar{X}_{\Sigma^{\prime}} \rightarrow \bar{X}_{\Sigma}$ and that $f^{*}\left(\bar{E}_{\Sigma}\right) \cong \bar{E}_{\Sigma^{\prime}}$ (hence $f^{*} c^{i}\left(\bar{E}_{\Sigma}\right)=$ $\left.c^{i}\left(\bar{E}_{\Sigma^{\prime}}\right)\right)$. Moreover, it is proven in [Har3] that the coherent sheaf $\tau_{*} \bar{E}_{\Sigma}$ is independent of the choice of $\Sigma$. One is therefore led to suspect the existence of a closer relationship between the characteristic classes of the vector bundles $\bar{E}_{\Sigma}$ and the topology of the BailyBorel compactification $\bar{X}$. In Theorem 11.8 and Theorem 13.2 we show that, at least for the variety $\bar{X}$, the original goal of constructing Chern numbers can be completely realized:

Theorem. Every Chern class $c^{i}(X)$ has a canonical lift $\bar{c}^{i} \in H^{2 i}(\bar{X} ; \mathbb{C})$ to the cohomology of the Baily-Borel compactification. Moreover, if $\tau: \bar{X}_{\Sigma} \rightarrow \bar{X}$ is any toroidal resolution of singularities then

$$
\tau^{*}\left(\bar{c}^{i}\right)=c^{i}\left(\bar{E}_{\Sigma}\right) \in H^{2 i}\left(\bar{X}_{\Sigma} ; \mathbb{C}\right) .
$$

It follows (§13.3) that the lifts $\bar{c}^{i}$ satisfy (1.1.1). In $\S 15$ we show that the homology image $\bar{c}^{*} \cap[\bar{X}] \in H_{*}(\bar{X})$ lies in integral homology and coincides with (MacPherson's) Chern class [Mac] of the constructible function which is 1 on $X$ and is 0 on $\bar{X}-X$.

1.2. Moreover, a similar result holds for any automorphic vector bundle. Let $\lambda: K \rightarrow$ $G L(V)$ be a representation of $K$ on some finite dimensional complex vector space $V$. By [Mu1], the automorphic vector bundle $E^{\prime}=(\Gamma \backslash G) \times_{K} V$ on $X$ has a particular extension $\bar{E}_{\Sigma}^{\prime}$ over any toroidal resolution $\bar{X}_{\Sigma}$. We show that each Chern class $c^{i}\left(E_{\Gamma}\right)$ has a canonical lift $\bar{c}^{i}\left(E^{\prime}\right) \in H^{2 i}(\bar{X} ; \mathbb{C})$ and that these lifts also satisfy the proportionality formula. Moreover, $\tau^{*}\left(\bar{c}^{i}\left(E^{\prime}\right)\right)=c^{i}\left(\bar{E}_{\Sigma}^{\prime}\right)$ and the image of $\bar{c}^{i}\left(E^{\prime}\right)$ in $\operatorname{Gr}_{2 i}^{W}(\bar{X} ; \mathbb{C})$ (the top graded piece of the weight filtration) is uniquely determined by this formula.

1.3. In $\S 16$ we consider the subalgebra $H_{\text {Chern }}^{*}(\bar{X} ; \mathbb{C})$ of the cohomology of the Baily-Borel compactification that is generated by the (above defined lifts of) Chern classes of certain "universal" automorphic vector bundles, and show that

Theorem. Suppose the Hermitian symmetric domain $D$ is a product of irreducible factors $G_{i} / K_{i}$ (where $K_{i}$ is a maximal compact subgroup of $G_{i}$ ), and that each $G_{i}$ is one of the following: $S p_{n}(\mathbb{R}), U(p, q), S O(2 n)$, or $S O(2, p)$ with $p$ odd or $p=2$. Then there is a (naturally defined) surjection $h: H_{\text {Chern }}^{*}(\bar{X} ; \mathbb{C}) \rightarrow H^{*}(\check{D} ; \mathbb{C})$ from this subalgebra to the cohomology of the compact dual symmetric space.

This result is compatible with the few known facts about the cohomology of the BailyBorel compactification. Charney and Lee [CL] have shown, when $D=S p_{2 n}(\mathbb{R}) / U(n)$ is the Siegel upper half space, and when $\Gamma=S p_{2 n}(\mathbb{Z})$ that the "stable" cohomology of $\bar{X}$ contains a polynomial algebra which coincides with the "stable" cohomology of the compact dual symmetric space $\check{D}$ (which is the complex Lagrangian Grassmannian). It is a general fact (cf. $\S 16)$ that the intersection cohomology $I H^{*}(\bar{X} ; \mathbb{C})$ contains a copy of $H^{*}(\check{D} ; \mathbb{C})$. 
1.4. Here are the main ideas behind the proof of Theorem 11.8. In [Hr2], Hirzebruch shows that the Chern classes of Hilbert modular varieties have lifts to the cohomology of the BailyBorel compactification because the tangent bundle has a trivialization in a neighborhood of each of the finitely many cusp points, cf. [ADS]. If $X=\Gamma \backslash D$ is a $\mathbb{Q}$-rank 1 locally symmetric space such that $\bar{X}$ is obtained from $X$ by adding finitely many cusps, then the tangent bundle is not necessarily trivial near each cusp, but it admits a connection which is flat near each cusp, and so the Chern forms vanish near each cusp, hence the Chern classes lift to the cohomology of the Baily-Borel compactification. A similar argument applies to arbitrary automorphic vector bundles (cf. [HZ1] §3.3.9).

In the general $\mathbb{Q}$-rank 1 case, the singular set of the Baily-Borel compactification $\bar{X}$ consists of finitely many disjoint smooth compact manifolds (rather than finitely many cusp points). If $Y$ denotes such a singular stratum, then it admits a neighborhood $\pi_{Y}: N_{Y} \rightarrow Y$ such that every slice $\pi_{Y}^{-1}(y) \cap X$ is diffeomorphic to a neighborhood of a cusp similar to the kind described above. It is then possible to construct a connection $\nabla$ (on the tangent bundle) which is "flat along each fiber $\pi_{Y}^{-1}(y)$ ". (We call this the "parabolic connection"; it is constructed in Section 10.) Moreover, within the neighborhood $N_{Y}$, each Chern form $\sigma^{i}(\nabla)$ is the pullback $\pi_{Y}^{*}\left(\sigma_{Y}^{i}\right)$ of a certain differential form $\sigma_{Y}^{i}$ on $Y$. Differential forms with this " $\pi$-fiber" or "control" property form a complex whose cohomology is the cohomology of $\bar{X}$, as discussed in Section 4. So the Chern form $\sigma^{i}(\nabla)$ determines a class $\bar{c}^{i}(\nabla) \in H^{2 i}(\bar{X} ; \mathbb{C})$. (In fact, even the curvature form is "controlled".)

1.5. In higher rank cases, there are more problems. If $Y_{1} \subset \bar{Y}_{2} \subset \bar{X}$ are singular strata of the Baily-Borel compactification, then it is possible to define a "parabolic" connection in a neighborhood $N\left(Y_{1}\right)$ of $Y_{1}$ whose curvature form is controlled relative to the tubular projection $\pi_{1}: N\left(Y_{1}\right) \rightarrow Y_{1}$. It is also possible to construct a "parabolic" connection in a neighborhood $N\left(Y_{2}\right)$ of $Y_{2}$ whose curvature form has the control property relative to the tubular projection $\pi_{2}: N\left(Y_{2}\right) \rightarrow Y_{2}$. However these two connections do not necessarily agree on the intersection $N\left(Y_{1}\right) \cap N\left(Y_{2}\right) \cap X$, nor do their curvature forms. When we patch these two connections together using a partition of unity, the curvature form of the resulting connection fails to be controlled. Nevertheless it is possible (as explained in Remark 11.3) to patch together connections of this type so as to obtain a connection whose curvature form $\Omega \in \operatorname{End}(V)$ differs from a controlled differential form by a nilpotent element $n \in \operatorname{End}(V)$ which commutes with $\Omega$ (cf. $\S 12.10$ ). (Here, $V$ is the representation of $K$ that gives rise to the automorphic vector bundle $E_{\Gamma}=(\Gamma \backslash G) \times_{K} V$ on $X=\Gamma \backslash G / K$.) This is enough to guarantee that the Chern forms of this "patched" connection are controlled differential forms (cf. Lemma 6.4). A standard argument shows that the resulting cohomology class is independent of the choices that were involved in the construction.

1.6. A number of interesting questions remain. We do not know whether the results on Chern classes which are described in this paper for Hermitian symmetric spaces may be extended to the "equal rank" case (when the real rank of $G$ and of $K$ coincide). We do not 
know if the lifts $\bar{c}^{i}\left(E^{\prime}\right) \in H^{2 i}(\bar{X} ; \mathbb{C})$ are integer or even rational cohomology classes. We do not know to what extent these lifts are uniquely determined by the properties (11.9), (13.2.1), (15.5). We do not know whether similar techniques can be applied to the Euler class of automorphic vector bundles (when such a class exists: see $\S 16$ ). We do not know whether the surjection $h$ of Theorem 16.4 admits a natural splitting. We expect that $\bar{c}^{*}\left(E^{\prime}\right)=0$ whenever the automorphic vector bundle $E^{\prime}$ arises from a representation $\lambda: K \rightarrow G L(V)$ which extends to a representation of $G$. If $\bar{E}_{\Gamma}^{\text {RBS }}$ denotes the canonical extension ([GT] $\S 9$ ) of the automorphic vector bundle $E$ over the reductive Borel-Serre ([Z1] $\S 4.2$ p. 190, [GHM] $\S 8)$ compactification $\nu: \bar{X}^{\mathrm{RBS}} \rightarrow \bar{X}$ then it is likely that $\nu^{*}\left(\bar{c}^{*}\left(E^{\prime}\right)\right)=c^{*}\left(\bar{E}_{\Gamma}^{\mathrm{RBS}}\right)$. We expect these results to have interesting applications to the study of the signature defect ([Hr2] $\S 3$, [ADS], [Mü], [St1]) and to variations of weight 1 (and some weight 2) Hodge structures ([Gr1], [Gr2]).

1.7. We would like to thank A. Borel, R. Bryant, D. Freed, R. Hain, M. Harris, R. MacPherson, and L. Saper for valuable conversations. We are grateful to S. Zucker for many suggestions and comments on an earlier draft of this paper. We are especially grateful to an anonymous referee for pointing out a mistake in an earlier version of this paper, and for his many helpful comments, suggestions, and corrections. Both authors would like to thank the Institute for Advanced Study in Princeton N.J. for its support and hospitality while this paper was prepared.

\subsection{List of symbols.}

$$
\begin{aligned}
& \S 2(2.1) Y<X,(2.2) T_{Y}(\epsilon), \pi_{Y}, \rho_{Y}, \partial T_{Y}(\epsilon) \\
& \S 3(3.1) s, s_{\epsilon}, s_{Z}^{\epsilon}, t_{\epsilon}^{Y}(3.2) B_{Y}^{\epsilon}(3.5) \epsilon Y, B_{n}^{\epsilon m} \\
& \S 4(4.1) \omega_{Y}, \mathcal{A}_{\pi}^{\bullet}(W),(4.2)[\omega] \\
& \S 5(5.1) \mathcal{A}^{i}(M, E), G, K, \mathfrak{g}, \mathfrak{k}, \lambda, \lambda^{\prime}, L_{h}, Y_{\dot{k}},(5.2) G \times_{K} V, \mathcal{A}_{\text {bas }}^{i}(G, V), i(Y), R_{k}, \nabla_{X}, \\
& \nabla=d+\omega, \Omega, \omega_{0},(5.5) \nabla^{\mathrm{Nom}},(5.6) J(g, x), \Phi_{J}, J_{0} \\
& \S 6(6.1)[\theta, \theta],(6.3) \sigma_{\nabla}^{f}, \sigma^{i}(\nabla),(6.5) \chi, a_{s}+a_{n}, C\left(a_{s}\right) \\
& \S 7 \text { (7.1) G, G, D, } \theta, K, D^{*}, \mathbf{P}, \mathcal{U}_{P}, L(P), \nu_{P}, G_{h}, G_{\ell}, \nu_{h}, \pi: D \rightarrow D_{1}(7.2) \mathbf{P}_{\mathbf{0}}, \Phi\left(\mathbf{S}_{\mathbf{0}}, G\right) \text {, } \\
& \Phi^{+}(7.3) \mathbf{P}_{\mathbf{1}} \prec \mathbf{P}_{\mathbf{2}}, D_{1} \prec D_{2}, \bar{P}, \overline{\mathcal{U}}, \overline{\bar{P}}, \overline{\overline{\mathcal{U}}}, Q^{b}, Q^{\sharp} \\
& \S 8(8.2) \lambda_{1},(8.3) P^{+}, P^{-}, j, c_{1}, J_{1} \\
& \S 9(9.1) \Gamma, q: D^{*} \rightarrow \bar{X},(9.4) D^{\mathrm{BS}}, D^{\dagger}(9.5) T\left(D_{1}\right) \\
& \S 10(10.1) \mathfrak{g}_{h}=\mathfrak{k}_{h} \oplus \mathfrak{p}_{h}, \mathfrak{g}_{\ell}=\mathfrak{k}_{\ell} \oplus \mathfrak{p}_{\ell}, \Phi(10.2) \Phi^{*}\left(\nabla_{1}\right) \\
& \S 11(11.2) \nabla_{Y}^{\mathrm{p}}(11.4) \mathbf{Z} \leq Y, B_{\mathbf{Z}}(x), \Phi_{\mathbf{Z}}^{*} \\
& \S 13(13.1) E^{\prime}, \bar{c}^{j}\left(E^{\prime}\right), \bar{X}_{\Sigma}, \bar{E}_{\Sigma}^{\prime}, \tau: \bar{X}_{\Sigma} \rightarrow \bar{X},(13.2) \bar{\nabla}_{\Sigma}^{\mathrm{p}} \text { (13.3) } \check{E}, \check{D}, G_{u}, \check{c}^{I}, \bar{c}^{I}, v(\Gamma) \\
& \S 14(14.3) \bar{\Phi}_{X Y}^{*}\left(\nabla_{Y}\right)(14.5) Z_{F}, C_{F} \subset \mathfrak{z}, \Sigma=\left\{\Sigma_{F}\right\}, \beta: D \rightarrow \check{D}, D_{F}, \Gamma_{F}^{\prime}, M_{F}^{\prime}, T_{F}, A_{F} \text {, } \\
& T_{F, \Sigma}, M_{F, \Sigma}^{\prime}, D_{F, \Sigma}, \varphi_{F, \Sigma}(14.6) \check{E}, E_{F}, E_{F}^{\prime}, \widetilde{E}, E_{F}^{A}, \bar{E}_{\Sigma}^{\prime}, \bar{E}_{F, \Sigma}^{\prime}, \psi_{\Sigma}, \widetilde{\Phi}_{F}, \Phi_{F},(14.7) \widetilde{\nabla} \\
& \nabla_{F}^{\prime}, \nabla_{F}^{A}, \nabla_{F, \Sigma}^{\prime} \\
& \S 15(15.1) \chi(W, F), f_{*}(F), c_{*}(W, F)(15.2) T_{\bar{Z}}(-\log D)
\end{aligned}
$$


$\S 16(16.1) E K, B K, E_{\lambda}, H_{\text {Chern }}^{*}, e\left(E_{\tilde{\lambda}}\right)$

\section{Control Data}

2.1. A weakly stratified space $W$ is a compact Hausdorff space with a decomposition into finitely many smooth manifolds $W=Y_{1} \cup Y_{2} \cup \ldots \cup Y_{r}$ (called the strata of $W$ ) which satisfy the axiom of the frontier: If $Y$ and $Z$ are strata and if $Z \cap \bar{Y} \neq \phi$ then $Z \subset \bar{Y}$; we write $Z<Y$ and say that $Z$ is incident to $Y$. The boundary $\partial \bar{Y}=\bar{Y}-Y=\cup_{Z<Y} Z$ of the stratum $Y$ is the union of all strata incident to $Y$. If $W=\bar{X}$ is the closure of a single stratum $X$ then we say that $X$ is the nonsingular part of $W$ and the other strata $Y<X$ are boundary or singular strata of $W$. Fix a positive real number $\epsilon>0$.

2.2. Definition. An $\epsilon$-system of control data on a weakly stratified space $W$ is a collection $\left\{T_{Y}(\epsilon), \pi_{Y}, \rho_{Y}\right\}$ indexed by the boundary strata $Y \subset W$, where

(1) $T_{Y}(\epsilon) \subset W$ is an open subset of $W$ containing $Y$,

(2) $T_{Y}(\epsilon) \cap T_{Z}(\epsilon)=\phi$ unless $Y<Z$ or $Z<Y$.

(3) The tubular projection $\pi_{Y}: T_{Y}(\epsilon) \rightarrow Y$ is a retraction of $T_{Y}(\epsilon)$ to $Y$ which is smooth on each stratum,

(4) $\pi_{Z} \pi_{Y}=\pi_{Z}$ whenever $Z<Y$ and both sides of the equation are defined,

(5) $\rho_{Y}: T_{Y}(\epsilon) \rightarrow[0, \epsilon)$ is a continuous "distance function", with $\rho_{Y}^{-1}(0)=Y$, such that the mapping $\left(\rho_{Y}, \pi_{Y}\right): T_{Y}(\epsilon) \rightarrow[0, \epsilon) \times Y$ is proper and its restriction to each stratum is a submersion,

(6) $\rho_{Z} \pi_{Y}=\rho_{Z}$ whenever $Z<Y$ and both sides of the equation are defined,

For $\tau \leq \epsilon$, write $T_{Y}(\tau)=\rho_{Y}^{-1}([0, \tau))$. By shrinking the neighborhood $T_{Y}(\epsilon)$ and scaling $\rho_{Y}$ if necessary, we may assume that each $\rho_{Y}$ is defined on a slightly larger neighborhood $T_{Y}\left(\epsilon^{\prime}\right)$ (where $\epsilon^{\prime}>\epsilon$ ) and that the "boundary" of $T_{Y}(\epsilon)$ is

$$
\partial T_{Y}(\epsilon)=\overline{T_{Y}(\epsilon)}-T_{Y}(\epsilon)=\overline{\rho_{Y}^{-1}(\epsilon)}=\rho_{Y}^{-1}(\epsilon) \cup \partial \bar{Y} .
$$

Such neighborhoods are illustrated in the following diagram.

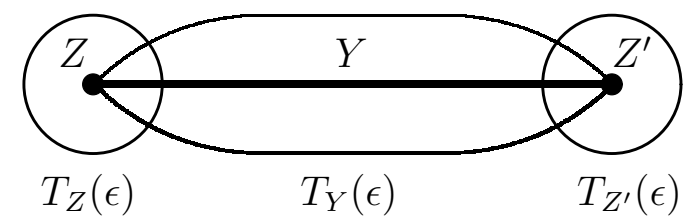

FIGURE 1. Tubular neighborhoods

If $W$ is the closure of a single stratum $X$ we extend this notation by setting $T_{X}(\epsilon)=X$, $\pi_{X}(x)=x$ and $\rho_{X}(x)=0$ for all $x \in X$. 
2.3. Any compact real or complex algebraic or analytic variety admits a Whitney stratification ([Ha1], [Ha2]). Any compact Whitney stratified subset of a smooth manifold admits a system of control data (see [Mat] or [Gi] Thm. 2.6). If $W$ is a compact Whitney stratified set and if the mappings $\left\{\pi_{Y}\right\}$ are preassigned so as to satisfy Conditions (3) and (4) above, then distance functions $\rho_{Y}$ may be found which are compatible with the mappings $\pi_{Y}$.

\section{Partition of Unity}

3.1. Throughout this paper we will fix a choice of a smooth nondecreasing function $s: \mathbb{R} \rightarrow$ $[0,1]$ so that $s(x)=0$ for all $x \leq 1 / 2$ and $s(x)=1$ for all $x \geq 3 / 4$. For any $\epsilon>0$ define $s_{\epsilon}(\rho)=s(\rho / \epsilon)$.

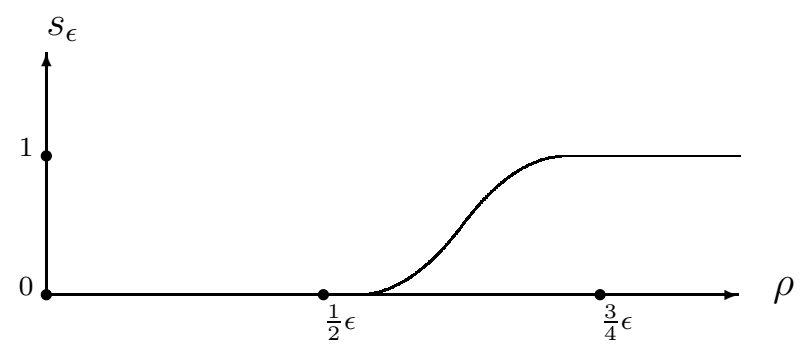

Figure 2. The function $s_{\epsilon}(\rho)$.

Fix $0<\epsilon \leq \epsilon_{0}$. Let $W$ be a weakly stratified space with an $\epsilon_{0}$ system of control data $\left\{T_{Y}\left(\epsilon_{0}\right), \pi_{Y}, \rho_{Y}\right\}$. For each stratum $Z \subset W$ define the modified distance function $s_{Z}^{\epsilon}$ : $T_{Z}(\epsilon) \rightarrow[0,1]$ by $s_{Z}^{\epsilon}(x)=s_{\epsilon}\left(\rho_{Z}(x)\right)$. Then $s_{Z}^{\epsilon}=0$ on $T_{Z}\left(\frac{\epsilon}{2}\right)$ and $s_{Z}^{\epsilon}=1$ near the edge $\partial T_{Z}(\epsilon)$ of the tubular neighborhood $T_{Z}(\epsilon)$.

For each stratum $Y \subset W$ define a smooth function $t_{\epsilon}^{Y}: Y \rightarrow \mathbb{R}$ as follows: If $y \in Y$ is not contained in the tubular neighborhood $T_{Z}(\epsilon)$ of any stratum $Z<Y$ then set $t_{\epsilon}^{Y}(y)=1$. Otherwise, there is a unique maximal collection of boundary strata $Z_{1}, Z_{2}, \ldots, Z_{r}$ such that $y \in T_{Z_{1}}(\epsilon) \cap T_{Z_{2}}(\epsilon) \cap \ldots \cap T_{Z_{r}}(\epsilon)$ and in this case, by (2.2) (Condition 2), these boundary strata form a flag $Z_{1}<Z_{2}<\ldots<Z_{r}$ (after possibly relabeling the indices). Define

$$
t_{\epsilon}^{Y}(y)=s_{Z_{1}}^{\epsilon}(y) \cdot s_{Z_{2}}^{\epsilon}(y) \ldots s_{Z_{r}}^{\epsilon}(y) .
$$

Then the function $t_{\epsilon}^{Y}: Y \rightarrow[0,1]$ is smooth and vanishes on

$$
\bigcup_{Z<Y} T_{Z}\left(\frac{\epsilon}{2}\right) \cap Y .
$$

Pull this up to a function $\pi_{Y}^{*} t_{\epsilon}^{Y}: T_{Y}(\epsilon) \rightarrow[0,1]$ by setting $\pi_{Y}^{*} t_{\epsilon}^{Y}(x)=t_{\epsilon}^{Y}\left(\pi_{Y}(x)\right)$. 
3.2. For each stratum $Y \subset W$, the product

$$
B_{Y}^{\epsilon}=\left(\pi_{Y}^{*} t_{\epsilon}^{Y}\right) \cdot\left(1-s_{Y}^{\epsilon}\right): T_{Y}(\epsilon) \rightarrow[0,1]
$$

is smooth, vanishes near $\partial T_{Y}(\epsilon)$, and also near $\partial \bar{Y}$ :

$$
x \in \bigcup_{Z<Y} T_{Z}\left(\frac{\epsilon}{2}\right) \Longrightarrow B_{Y}^{\epsilon}(x)=0 .
$$

Hence $B_{Y}^{\epsilon}$ admits an extension to $W$ which is defined by setting

$$
B_{Y}^{\epsilon}(x)=0 \text { if } x \notin T_{Y}(\epsilon) .
$$

This extension is smooth on each statum of $W$ and satisfies the following conditions whenever $Z<Y$ :

$$
\begin{gathered}
B_{Z}^{\epsilon} \pi_{Y}(x)=B_{Z}^{\epsilon}(x) \text { for all } x \in T_{Y}\left(\epsilon_{0}\right) \\
B_{Y}^{\epsilon}\left(\pi_{Y}(x)\right)=B_{Y}^{\epsilon}(x)=t_{\epsilon}^{Y}\left(\pi_{Y}(x)\right) \text { for all } x \in T_{Y}(\epsilon / 2) \\
B_{Y}^{\epsilon}(x)=1 \text { for all } x \in T_{Y}(\epsilon / 2)-\bigcup_{Z<Y} T_{Z}(\epsilon) .
\end{gathered}
$$

3.3. Lemma. For every stratum $Y \subset W$ and for every point $y \in \bar{Y}$ we have

$$
B_{Y}^{\epsilon}(y)+\sum_{Z<Y} B_{Z}^{\epsilon}(y)=1
$$

3.4. Proof. It suffices to verify (3.3.1) for $y \in Y$. Then $B_{Y}^{\epsilon}(y)=t_{\epsilon}^{Y}(y)$. If $y$ is not in any tubular neighborhood $T_{Z}(\epsilon) \cap Y$ (for $Z<Y$ ) then $t_{\epsilon}^{Y}(y)=1$ and $1-s_{Z}^{\epsilon}(y)=0$. Otherwise, let $\left\{Z_{1}, Z_{2}, \ldots Z_{r}\right\}$ be the collection of strata for which $Z_{i}<Y$ and $y \in T_{Z_{i}}(\epsilon)$. By relabeling the indices, we may assume that $Z_{1}<Z_{2}<\ldots Z_{r}<Y$ form a flag of strata. The nonzero terms in the sum (3.3.1) involve only the functions $s_{1}, s_{2}, \ldots, s_{r}$ (where $s_{i}=s_{Z_{i}}^{\epsilon}$ ) and can be written:

$$
\left(1-s_{1}\right)+s_{1}\left(1-s_{2}+s_{2}\left(\ldots+s_{r-1}\left(1-s_{r}+s_{r}\right) \ldots\right)\right)=1
$$

3.5. In $\S 11.2$ we will construct a connection on a modular variety by induction, patching together connections which have been previously defined on neighborhoods of boundary strata. For each step of this induction we will need a different partition of unity, which is obtained from (3.3.1) by shrinking the parameter $\epsilon$. The purpose of this subsection is to construct the family of partitions of unity.

Let $W$ be a weakly stratified space with an $\epsilon_{0}>0$ system of control data, $\left\{T_{Y}\left(\epsilon_{0}\right), \pi_{Y}, \rho_{Y}\right\}$. Suppose each stratum $Y$ is a complex manifold and define

$$
\epsilon Y=\epsilon_{0} / 2^{\operatorname{dim}_{\mathbb{C}}(Y)} .
$$




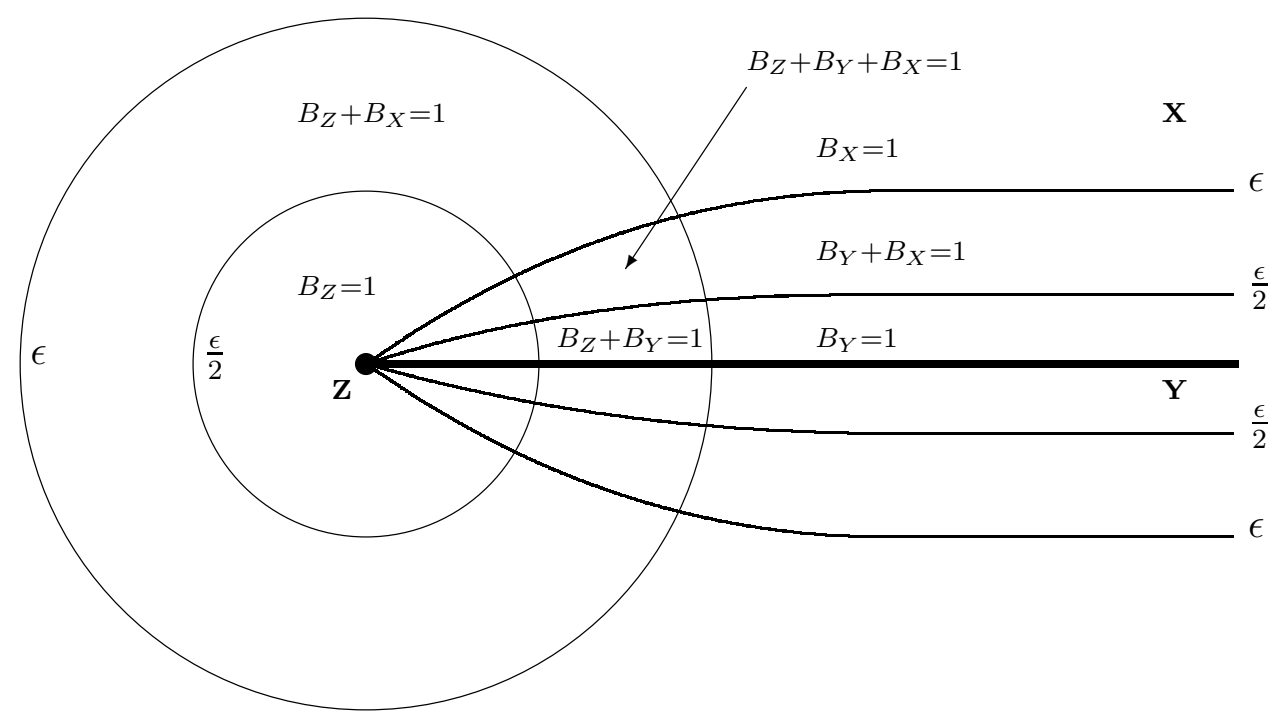

Figure 3. Partition of Unity for fixed $\epsilon$

(The complex structure is irrelevant to this construction and is only introduced so as to agree with the later sections in this paper.) By Lemma 3.3 for every point $x \in Y$ we have

$$
\sum_{Z \leq Y} B_{Z}^{\epsilon Y}(x)=1
$$

By (3.2.4) and (3.2.5) the same equation holds, in fact for all $x \in T_{Y}\left(\frac{\epsilon Y}{2}\right)$.

Let $x \in W$. Then there is a maximal collection of strata $Y_{1}, Y_{2}, \ldots, Y_{r}$ such that

$$
x \in T_{Y_{1}}\left(\epsilon_{0}\right) \cap T_{Y_{2}}\left(\epsilon_{0}\right) \cap \ldots \cap T_{Y_{r}}\left(\epsilon_{0}\right) .
$$

These strata form a partial flag which (we may assume) is given by $Y_{1}<Y_{2}<\cdots<Y_{r}$. Set

$$
B_{n}^{\epsilon m}=B_{Y_{n}}^{\epsilon Y_{m}} \text { and } \pi_{m}(x)=\pi_{Y_{m}}(x) .
$$

In the following lemma we assume $1 \leq m, n, m^{\prime}, n^{\prime} \leq r$, that $m \geq n$ and that $m^{\prime} \geq n^{\prime}$.

3.6. Lemma. If $B_{n}^{\epsilon m}\left(\pi_{n}(x)\right) \neq 0$ then $B_{n^{\prime}}^{\epsilon m^{\prime}}(x)=0$ for all $n^{\prime}<n$ and for all $m^{\prime}>m$. If $B_{n}^{\epsilon m}(x) \neq 0$ then $B_{n^{\prime}}^{\epsilon m^{\prime}}\left(\pi_{n^{\prime}}(x)\right)=0$ for all $n^{\prime}>n$ and for all $m^{\prime}<m$.

3.7. Proof. If $B_{n}^{\epsilon m}\left(\pi_{n}(x)\right) \neq 0$ then $\pi_{n}(x) \notin T_{n^{\prime}}(\epsilon m / 2) \supset T_{n^{\prime}}\left(\epsilon m^{\prime}\right)$ by (3.2.2). Therefore $B_{n^{\prime}}^{\epsilon m^{\prime}}\left(\pi_{n}(x)\right)=0$ by (3.2.3). Hence $B_{n^{\prime}}^{\epsilon m^{\prime}}(x)=0$ by (3.2.4). The second statement is the contrapositive of the first.

\section{Controlled differential FORMS}

4.1. Suppose $W$ is a stratified space with a fixed $\epsilon$-system of control data $\left\{T_{Y}(\epsilon), \pi_{Y}, \rho_{Y}\right\}$. Define a controlled differential form $\omega$ to be a collection $\omega=\left\{\omega_{Y} \in \mathcal{A}^{*}(Y ; \mathbb{C})\right\}$ of smooth 
differential forms (with complex coefficients) on the strata $Y$ of $W$, which satisfy the following compatibility condition whenever $Z<Y$ : There exists a neighborhood $T(\omega) \subset T_{Z}(\epsilon)$ of $Z$ such that

$$
\omega_{Y}\left|(Y \cap T(\omega))=\pi_{Y Z}^{*}\left(\omega_{Z}\right)\right|(Y \cap T(\omega)) .
$$

(Here, $\pi_{Y Z}$ denotes the restriction $\pi_{Z} \mid Y \cap T_{Z}(\epsilon)$.) We refer to equation (4.1.1) as the control condition. If $\omega=\left\{\omega_{Y}\right\}$ is a controlled differential form, define its differential to be the controlled differential form $d \omega=\left\{d \omega_{Y}\right\}$. Let $\mathcal{A}_{\pi}^{\bullet}(W)$ denote the complex of controlled differential forms and let $H_{\pi}^{*}(W)$ denote the resulting cohomology groups. These differential forms are analogous to the $\pi$-fiber cocycles in [Go1]. Recall [V] the following theorem of A. Verona.

4.2. Theorem. The inclusion $\mathbb{C} \rightarrow \mathcal{A}_{\pi}^{\bullet}(W)$ of the constant functions into the complex of controlled differential forms induces an isomorphism

$$
H^{i}(W ; \mathbb{C}) \cong H_{\pi}^{i}(W)
$$

for all $i$. The restriction $i^{*}[\omega] \in H^{*}(\bar{Y})$ of the cohomology class $[\omega]$ represented by a closed, controlled differential form $\omega=\left\{\omega_{Y}\right\}_{Y \subset W}$ to the closure $\bar{Y}$ of a single stratum is given by the controlled differential form $\left\{\omega_{Z}\right\}_{Z \subset \bar{Y}}$ (where $i: \bar{Y} \rightarrow W$ denotes the inclusion).

4.3. Outline of Proof. Since the control condition is local, the controlled differential forms $\mathcal{A}_{\pi}^{i}(W)$ are the global sections of a sheaf $\mathcal{A}_{\pi}^{i}$ of controlled differential forms (which is obtained by first restricting controlled forms to open sets and then sheafifying the resulting presheaf). This sheaf is a module over the sheaf of controlled functions and hence is fine. Let $Z$ be a stratum in $W$, fix $x \in Z$ and let $B \subset Z$ be a small open ball containing the point $x$. Let

$$
U=\pi_{Z}^{-1}(B)=\coprod_{Y \geq Z} \pi_{Y Z}^{-1}(B)
$$

be the resulting "basic" open neighborhood of $x \in W$ ([Mat], [GM1] §1.1, [GM2] §1.1, [GM3] $\S 1.4$ ). If $\omega=\left\{\omega_{Y}\right\}$ is a controlled differential form (4.1.1) in the whole neighborhood $U$, then it is completely determined by the smooth differential form $\omega_{Z} \in \mathcal{A}^{*}(B)$. Hence, the Poincaré lemma for the ball $B \subset Z$ implies the Poincaré lemma for the stalk $\mathcal{A}_{\pi, x}^{*}$ at $x$ of the complex of controlled differential forms. This shows that the inclusion of the constant sheaf $\mathbb{C}_{W} \rightarrow \mathcal{A}_{\pi}^{*}$ into the complex of sheaves of controlled differential forms is a quasiisomorphism and hence induces an isomorphism on hypercohomology. The statement about the restriction to $H^{*}(\bar{Y})$ follows immediately.

4.4. Suppose the stratified space $W$ is the closure of a single stratum $X$. Then a smooth differential form $\omega_{X} \in \mathcal{A}^{I}(X)$ is the $X$-component of a controlled differential form $\omega \in$ $\mathcal{A}_{\pi}^{i}(W)$ if and only if for each stratum $Y$ there exists a neighborhood $U_{Y}$ of $Y$ such that for each point $p \in U_{Y} \cap X$ and for every tangent vector $v \in T_{p} X$ the following condition holds:

$$
\text { If } d \pi(p)(v)=0 \text { then } i_{v} \omega=0
$$


where $i_{v}$ denotes the contraction with $v$. If (4.4.1) holds, then the controlled form $\omega$ is uniquely determined by $\omega_{X}$.

Now suppose that $W=\bar{X}$ is a compact subanalytic Whitney stratified subset of some (real) analytic manifold, and that $\tau: \widetilde{W} \rightarrow W$ is a (subanalytic) resolution of singularities (cf. [Hi1], [Hi2]). This means that $\widetilde{W}$ is a smooth compact subanalytic manifold, the mapping $\tau$ is subanalytic, its restriction $\tau^{-1}(X) \rightarrow X$ to $\tau^{-1}(X)$ is a diffeomorphism, and $\tau^{-1}(X)$ is dense in $\widetilde{W}$. Let $\omega_{X} \in \mathcal{A}^{i}(X)$ be a controlled differential form on $W$.

4.5. Lemma. Suppose the differential form $\tau^{*}\left(\omega_{X}\right)$ is the restriction of a smooth closed differential form $\widetilde{\omega} \in \mathcal{A}^{i}(\widetilde{W})$. Let $[\widetilde{\omega}] \in H^{i}(\widetilde{W} ; \mathbb{R})$ and $\left[\omega_{X}\right] \in H^{i}(W ; \mathbb{R})$ denote the corresponding cohomology classes. Then $[\widetilde{\omega}]=\tau^{*}\left(\left[\omega_{X}\right]\right)$.

4.6. Proof. The cohomology classes $[\widetilde{\omega}]$ and $\left[\omega_{X}\right]$ are determined by their integrals over subanalytic cycles by [Ha1], [Ha2]. Any subanalytic cycle $\xi \in C_{i}(\widetilde{W} ; \mathbb{R})$ may be made transverse (within its homology class) to the "exceptional divisor" $\tau^{-1}(W-X)$ ([GM3] $\S 1.3 .6)$. Then

$$
\int_{\xi} \widetilde{\omega}=\int_{\xi \cap \tau^{-1}(X)} \widetilde{\omega}=\int_{\tau(\xi) \cap X} \omega_{X}=\int_{\tau(\xi)} \omega_{X}
$$

\section{Homogeneous Vector BUNDLES}

5.1. If $M$ is a smooth manifold and $E \rightarrow M$ is a smooth vector bundle, let $\mathcal{A}^{i}(M, E)$ denote the space of smooth differential $i$-forms with values in $E$. Throughout this section, $K$ denotes a closed subgroup of a connected Lie group $G$ with Lie algebras $\mathfrak{k} \subset \mathfrak{g}$ and with quotient $D=G / K$. We fix a representation $\lambda: K \rightarrow G L(V)$ on some finite dimensional (real or complex) vector space $V$. Write $\lambda^{\prime}: \mathfrak{k} \rightarrow \operatorname{End}(V)$ for its derivative at the identity, and note that its derivative at a general point $g \in K$ is given by

$$
d \lambda(h)\left(\left(L_{h}\right)_{*}(\dot{k})\right)=\lambda(h) \lambda^{\prime}(\dot{k}) \in \operatorname{End}(V)
$$

for any $\dot{k} \in \mathfrak{k}$, where $L_{h}: K \rightarrow K$ is multiplication from the left by $h \in K$. The quotient mapping $q: G \rightarrow D=G / K$ is a principal $K$-bundle. The fundamental vertical vectorfields $Y_{\dot{k}}(g)=L_{g *}(\dot{k})$ (for $\left.\dot{k} \in \mathfrak{k}\right)$ determine a canonical trivialization, $\operatorname{ker}(d q) \cong G \times \mathfrak{k}$.

5.2. The representation $\lambda: K \rightarrow G L(V)$ determines an associated homogeneous vector bundle $E=G \times_{K} V$ on $D=G / K$, which consists of equivalence classes $[g, v]$ of pairs $(g, v) \in G \times V$ under the equivalence relation $(g k, v) \sim(g, \lambda(k) v)$ for all $g \in G, k \in K$, $v \in V$. It admits the homogeneous $G$ action given by $g^{\prime} \cdot[g, v]=\left[g^{\prime} g, v\right]$. Smooth sections $\tilde{s}$ of $E$ may be identified with smooth mappings $s: G \rightarrow V$ such that

$$
s(g k)=\lambda\left(k^{-1}\right) s(g)
$$

by $\tilde{s}(g K)=[g, s(g)] \in E$. Then (5.2.1) implies

$$
d s(g)\left(L_{g *}(\dot{k})\right)=-\lambda^{\prime}(\dot{k}) s(g)
$$


for all $g \in G$ and $\dot{k} \in \mathfrak{k}$.

Similarly we identify smooth differential forms $\tilde{\eta} \in \mathcal{A}^{i}(D, E)$ (with values in the vector bundle $E$ ) with differential forms $\eta \in \mathcal{A}_{\text {bas }}^{i}(G, V)$ which are "basic," meaning they are both $K$-equivariant $\left(R_{k}^{*}(\eta)=\lambda(k)^{-1} \eta\right.$ for all $\left.k \in K\right)$ and horizontal $\left(i\left(Y_{\dot{k}}\right) \eta=0\right.$ for all $\left.\dot{k} \in \mathfrak{k}\right)$. Here, $i(Y)$ denotes the interior product with the vectorfield $Y$ and $R_{k}(g)=g k$ for $g \in G$.

A connection $\nabla$ on $E$ is determined by a connection 1-form $\omega \in \mathcal{A}^{1}(G$, $\operatorname{End}(V))$ which satisfies $\omega\left(L_{g *}(\dot{k})\right)=\lambda^{\prime}(\dot{k})$ and $R_{k}^{*}(\omega)=\operatorname{Ad}\left(\lambda\left(k^{-1}\right)\right) \omega$ for any $\dot{k} \in \mathfrak{k}, k \in K$, and $g \in G$. The covariant derivative $\nabla_{X}$ with respect to a vectorfield $X$ on $D$ acts on sections $s: G \rightarrow V$ satisfying (5.2.1) by

$$
\nabla_{X} s(g)=d s(g)(\tilde{X}(g))+\omega_{g}(\tilde{X}(g))(s(g))
$$

where $\tilde{X}$ is any lift of $X$ to a smooth vectorfield on $G$. We write $\nabla=d+\omega$. The curvature form $\Omega \in \mathcal{A}^{2}(D, \operatorname{End}(E))$ takes values in the vector bundle $\operatorname{End}(E)=G \times_{K} \operatorname{End}(\mathrm{V})$ and it will be identified with the "basic" 2 -form $\Omega \in \mathcal{A}_{\text {bas }}^{2}(G$, End $(V))$ which assigns to tangent vectors $X, Y \in T_{g} G$ the endomorphism

$$
\Omega(X, Y)=d \omega(X, Y)+[\omega(X), \omega(Y)]
$$

If the Lie bracket is extended in a natural way to Lie algebra-valued 1 -forms, then it turns out $([\mathrm{BGV}] \S 1.12)$ that $[\alpha, \alpha](X, Y)=2[\alpha(X), \alpha(Y)]$ so we may express the curvature form as $\Omega=d \omega+\frac{1}{2}[\omega, \omega]$.

The connection $\nabla=d+\omega$ is $G$-invariant iff $L_{g}^{*}(\omega)=\omega$, in which case it is determined (on the identity component $G^{0}$ ) by its value $\omega_{0}: \mathfrak{g} \rightarrow \operatorname{End}(V)$ at the identity. Using [Wa] or $[\mathrm{KN}]$ Chapt. II Thm. 11.5. it is easy to verify the following result.

5.3. Proposition. Suppose $G$ is a connected Lie group and $K$ is a closed subgroup. Then the $G$-invariant connections on the homogeneous vector bundle $E=G \times_{K} V$ are given by linear mappings $\omega_{0}: \mathfrak{g} \rightarrow \operatorname{End}(V)$ such that

(1) $\omega_{0}(\dot{k})=\lambda^{\prime}(\dot{k})$ for all $\dot{k} \in \mathfrak{k}$ and

(2) $\omega_{0}([\dot{g}, \dot{k}])=\left[\omega_{0}(\dot{g}), \lambda^{\prime}(\dot{k})\right]$ for all $\dot{g} \in \mathfrak{g}$ and all $\dot{k} \in \mathfrak{k}$.

Moreover the curvature $\Omega \in \mathcal{A}^{2}(G, \operatorname{End}(V))$ of such a connection is the left-invariant "basic" differential form whose value $\Omega_{0}$ at the identity is given by

$$
\Omega_{0}(\dot{g}, \dot{h})=\left[\omega_{0}(\dot{g}), \omega_{0}(\dot{h})\right]-\omega_{0}([\dot{g}, \dot{h}])
$$

for any $\dot{g}, \dot{h} \in \mathfrak{g}$. The connection is flat iff $\omega_{0}$ is a Lie algebra homomorphism.

5.4. Example. Suppose the representation $\lambda: K \rightarrow G L(V)$ is the restriction of a representation $\tilde{\lambda}: G \rightarrow G L(V)$. Then we obtain a flat connection with $\omega_{0}(\dot{g})=\tilde{\lambda}^{\prime}(\dot{g})$ for $\dot{g} \in \mathfrak{g}$. 
5.5. Example. A connection in the principal bundle $G \rightarrow D$ is given by a connection 1form $\theta \in \mathcal{A}^{1}(G, \mathfrak{k})$ such that $R_{k}^{*}(\theta)=A d\left(k^{-1}\right)(\theta)$ and $\theta\left(Y_{\dot{k}}\right)=\dot{k}$ (for any $k \in K$ and any fundamental vectorfield $Y_{\dot{k}}$ ). It determines a connection $\nabla=d+\omega$ in the associated bundle $E=G \times_{K} V$ by $\omega(X)=\lambda^{\prime}(\theta(X))$. The principal connection $\theta$ is $G$-invariant iff $L_{g}^{*}(\theta)=\theta$ in which case it is determined by its value $\theta_{0}: \mathfrak{g} \rightarrow \mathfrak{k}$ at the identity. Conversely, by [No], any linear mapping $\theta_{0}: \mathfrak{g} \rightarrow \mathfrak{k}$ determines a $G$-invariant principal connection iff $\operatorname{ker}\left(\theta_{0}\right)$ is preserved under the adjoint action of $K$. If $K$ is a maximal compact subgroup of $G$ then the Cartan decomposition $\mathfrak{g}=\mathfrak{k} \oplus \mathfrak{p}$ determines a canonical $G$ invariant connection in the principal bundle $G \rightarrow G / K$, and hence a connection $\nabla^{\text {Nom }}$ on $E$ which we refer to as the Nomizu connection. It is given by $\omega_{0}(\dot{k}+\dot{p})=\lambda^{\prime}(\dot{k})$ for $\dot{k} \in \mathfrak{k}$ and $\dot{p} \in \mathfrak{p}$. By (5.3.1), its curvature is given by

$$
\Omega\left(L_{g *}\left(\dot{g}_{1}\right), L_{g *}\left(\dot{g}_{2}\right)\right)=-\lambda^{\prime}\left(\left[\dot{p}_{1}, \dot{p}_{2}\right]\right)
$$

(where $\dot{g}_{i}=\dot{k}_{i}+\dot{p}_{i} \in \mathfrak{k} \oplus \mathfrak{p}$ ), since $[\mathfrak{k}, \mathfrak{p}] \subset \mathfrak{p}$ and $[\mathfrak{p}, \mathfrak{p}] \subset \mathfrak{k}$.

5.6. There is a further description of homogeneous vector bundles on $D$ which are topologically trivial. Let $E=G \times_{K} V$ be a homogeneous vector bundle corresponding to a representation $\lambda: K \rightarrow G L(V)$ of $K$. A (smooth) automorphy factor $J: G \times D \rightarrow G L(V)$ for $E$ (or for $\lambda$ ) is a (smooth) mapping such that

(1) $J\left(g g^{\prime}, x\right)=J\left(g, g^{\prime} x\right) J\left(g^{\prime}, x\right)$ for all $g, g^{\prime} \in G$ and for all $x \in D$

(2) $J\left(k, x_{0}\right)=\lambda(k)$ for all $k \in K$.

It follows (by taking $g=1$ ) that $J(1, x)=I$. The automorphy factor $J$ is determined by its values $J\left(g, x_{0}\right)$ at the basepoint: any smooth mapping $j: G \rightarrow G L(V)$ such that $j(g k)=j(g) \lambda(k)$ (for all $k \in K$ and all $g \in G$ ) extends in a unique way to an automorphy factor $J: G \times D \rightarrow G L(V)$ for $E$, namely

$$
J\left(g, h x_{0}\right)=j(g h) j(h)^{-1} .
$$

An automorphy factor $J$, if it exists, determines a (smooth) trivialization

$$
\Phi_{J}: G \times_{K} V \rightarrow(G / K) \times V
$$

by $[g, v] \mapsto\left(g K, J\left(g, x_{0}\right) v\right)$. This trivialization is $G$-equivariant with respect to the following $J$-automorphic action of $G$ on $(G / K) \times V$ :

$$
g \cdot(x, v)=(g x, J(g, x) v) .
$$

Conversely, any smooth trivialization $\Phi: E \cong(G / K) \times V$ of the homogeneous vector bundle $E$ determines a unique automorphy factor $J$ such that $\Phi=\Phi_{J}$. A trivialization of $E$ (if one exists) allows one to identify smooth sections $s$ of $E$ with smooth mappings $r: D \rightarrow V$. If the trivialization is given by an automorphy factor $J$ and the smooth section $s$ of $E$ is given by a $K$-equivariant mapping $s: G \rightarrow V$ as in (5.2.1) then the corresponding smooth mapping is

$$
r(g K)=J\left(g, x_{0}\right) s(g)
$$


which is easily seen to be well defined. Sections $s$ which are invariant under $\gamma \in G$ correspond to functions $r$ such that $r(\gamma x)=J(\gamma, x) r(x)$ for all $x \in D$.

If $D=G / K$ is Hermitian symmetric of noncompact type then the canonical automorphy factor $(\S 8.3) J_{0}: G \times D \rightarrow \mathbf{K}(\mathbb{C})$ determines an automorphy factor $J=\lambda_{\mathbb{C}} \circ J_{0}$ for every homogeneous vector bundle $E=G \times_{K} V$, where $\lambda_{\mathbb{C}}: \mathbf{K}(\mathbb{C}) \rightarrow \mathrm{GL}(V)$ is the complexification of $\lambda$.

5.7. If $J_{1}, J_{2}$ are two automorphy factors for $E$, then the mapping $\phi: D \times V \rightarrow D \times V$ which is given by $\phi\left(g x_{0}, v\right)=\left(g x_{0}, J_{1}\left(g, x_{0}\right) J_{2}\left(g, x_{0}\right)^{-1} v\right)$ is a well defined $G$-equivariant isomorphism of trivial bundles, where $G$ acts on the domain via the $J_{1}$-automorphic action and $G$ acts on the target via the $J_{2}$-automorphic action.

5.8. (The following fact will be used in $\S 10.4$.) Suppose that $J: G \times D \rightarrow G L(V)$ is an automorphy factor for $E$. Let $\nabla=d+\omega$ be a connection on $E$. The trivialization $\Phi_{J}$ of $E$ (5.6.2) determines a connection $\nabla^{J}=d+\eta$ on the trivial bundle $D \times V$ (with $\left.\eta \in \mathcal{A}^{1}(D, \operatorname{End}(V))\right)$ as follows: if $r(g K)=J\left(g, x_{0}\right) s(g)$ as in (5.6.4) then $\nabla_{q_{*} X}^{J} r(g K)=$ $J\left(g, x_{0}\right) \nabla_{X} s(g)$ (for any $\left.X \in T_{g} G\right)$. It follows from (5.1.1) that the connection 1-forms are related by

$$
\eta\left(q_{*}(X)\right)=J\left(g, x_{0}\right) \omega(X) J\left(g, x_{0}\right)^{-1}-\left(d_{X} J\left(g, x_{0}\right)\right) J\left(g, x_{0}\right)^{-1} .
$$

\section{LEMMAS ON CURVATURE}

6.1. Suppose $E=G \times{ }_{K} V$ is a homogeneous vector bundle over $D=G / K$ arising from a representation $K \rightarrow G L(V)$ of a closed subgroup $K$ of a Lie group $G$ on a complex vector space $V$. If $\theta \in \mathcal{A}^{1}(G, \operatorname{End}(V))$ is a Lie algebra-valued 1 -form, denote by $[\theta, \theta]$ the Lie algebra valued 2-form $(X, Y) \mapsto 2[\theta(X), \theta(Y)]$ (cf [BGV] $\S 1.12)$. The proof of the following lemma is a direct but surprisingly tedious computation.

6.2. Lemma. Suppose $\left\{f_{1}, f_{2}, \ldots, f_{n}\right\}$ form a smooth partition of unity on $D$, that $i s \quad 0 \leq$ $f_{i}(x) \leq 1$ and $\sum_{i=1}^{n} f_{i}(x)=1$ for all $x \in D$. Let $\nabla_{i}=d+\omega_{i}$ be connections on $E$ with curvature forms $\Omega_{i}$ for $1 \leq i \leq n$. Let $\nabla=\sum_{i=1}^{n} f_{i} \nabla_{i}$ be the connection with connection form $\omega=\sum_{i=1}^{n} f_{i} \omega_{i}$. Then the curvature $\Omega$ of $\nabla$ is given by

$$
\Omega=\sum_{i=1}^{n} f_{i} \Omega_{i}-\frac{1}{2} \sum_{i<j} f_{i} f_{j}\left[\omega_{i}-\omega_{j}, \omega_{i}-\omega_{j}\right]+\sum_{i=1}^{n-1} d f_{i} \wedge\left(\omega_{i}-\omega_{n}\right) .
$$

(Even if the $\nabla_{i}$ are flat and the $f_{i}$ are constant, the connection $\nabla$ is not necessarily flat.)

6.3. Let $\mathcal{E}$ be a complex vector space and let $f: \mathcal{E} \rightarrow \mathbb{C}$ be a homogeneous polynomial of degree $k$. The polarization of $f$ is the unique symmetric $k$-linear form $P: \mathcal{E} \times \mathcal{E} \times \ldots \times \mathcal{E} \rightarrow \mathbb{C}$ such that $f(x)=P(x, x, \ldots, x)$ for all $x \in \mathcal{E}$. If $N \subset \mathcal{E}$ is a vectorsubspace such that $f(x+n)=f(x)$ for all $x \in \mathcal{E}$ and all $n \in N$ then the polarization $P$ satisfies

$$
P\left(x_{1}+n_{1}, x_{2}+n_{2}, \ldots, x_{k}+n_{k}\right)=P\left(x_{1}, x_{2}, \ldots, x_{k}\right)
$$


for all $x_{1}, \ldots, x_{k} \in \mathcal{E}$ and all $n_{1}, \ldots, n_{k} \in N$.

Now let $K \rightarrow G L(V)$ be a representation on a complex vector space $V$ as above, and let $f: \mathcal{E}=\operatorname{End}(V) \rightarrow \mathbb{C}$ be a homogeneous polynomial of degree $k$, which is invariant under the adjoint action $K \rightarrow G L(\mathfrak{k}) \rightarrow G L(\operatorname{End}(V))$ of $K$. Then the polarization $P$ of $f$ is also $\operatorname{Ad}_{K}$-invariant. If $\nabla$ is a connection on $E=G \times{ }_{K} V$ with curvature form $\Omega \in \mathcal{A}^{2}(G$, $\operatorname{End}(V))$ then the characteristic form associated to $f$ is

$$
\sigma_{\nabla}^{f}\left(X_{1}, Y_{1}, \ldots, X_{k}, Y_{k}\right)=P\left(\Omega\left(X_{1}, Y_{1}\right), \ldots, \Omega\left(X_{k}, Y_{k}\right)\right) \in \mathcal{A}^{2 k}(G, \mathbb{C}) .
$$

It is "basic" (cf. §5.2) and hence descends uniquely to a differential form on $D$ which we also denote by $\sigma_{\nabla}^{f} \in \mathcal{A}^{2 k}(D, \mathbb{C})$. Throughout this paper we shall be concerned only with homogeneous polynomials $f: \operatorname{End}(V) \rightarrow \mathbb{C}$ which are invariant under the full adjoint action of $G L(V)$ and which we shall refer to as a Ad-invariant polynomials. When $f(x)$ is the $i$-th elementary symmetric function in the eigenvalues of $x$, the resulting characteristic form is the $i$-th Chern form and it will be denoted $\sigma^{i}(\nabla)$.

6.4. Lemma. Let $V$ be a complex vector space, $H=\mathrm{GL}(V)$ and $\mathfrak{h}=\operatorname{End}(V)$. Let $x, n \in \mathfrak{h}$ and suppose that $[x, n]=0$ and that $n$ is nilpotent. Then for any Ad-invariant polynomial $f: \mathfrak{h} \rightarrow \mathbb{C}$ we have:

$$
f(x+n)=f(x) .
$$

6.5. Proof. Let $\mathfrak{t} \subset \mathfrak{h}$ be the Lie algebra of a Cartan subgroup $T \subset H$ and let $W$ denote its Weyl group. The adjoint quotient mapping $\chi: \mathfrak{h} \rightarrow \mathfrak{t} / W$ associates to any $a \in \mathfrak{h}$ the $W$-orbit $C\left(a_{s}\right) \cap \mathfrak{t}$ where $a=a_{s}+a_{n}$ is the Jordan decomposition of $a$ into its semisimple and nilpotent parts (with $\left[a_{s}, a_{n}\right]=0$ ), and where $C\left(a_{s}\right)$ denotes the conjugacy class of $a_{s}$ in $\mathfrak{h}$, cf. $[\mathrm{Sp}]$. (The value $\chi(a)$ may be interpreted as the coefficients of the characteristic polynomial of $a \in \operatorname{End}(V)$.) Since $x$ and $n$ commute, $(x+n)_{s}=x_{s}+n_{s}=x_{s}$, hence $\chi(x+n)=\chi(x)$. But every Ad-invariant polynomial $f: \mathfrak{h} \rightarrow \mathbb{C}$ factors through $\chi$, hence $f(x+n)=f(x)$.

\section{Hermitian Symmetric Spaces}

7.1. Throughout the remainder of this paper, algebraic groups will be denoted by boldface type and the associated group of real points will be denoted in Roman. Fix a reductive algebraic group $\mathbf{G}$ which is defined over $\mathbb{Q}$ and which (for convenience only) is assumed to be connected and simple over $\mathbb{Q}$. Suppose $G=\mathbf{G}(\mathbb{R})$ acts as the identity component of the group of automorphisms of a Hermitian symmetric space $D$. A choice of basepoint $x_{0} \in D$ determines a Cartan involution $\theta: G \rightarrow G$, a maximal compact subgroup $K=G^{\theta}=\operatorname{Stab}_{G}\left(x_{0}\right)$ and a diffeomorphism $G / K \cong D$. Let $D^{*}$ denote the Satake partial compactification of $D$, in other words, the union of $D$ and all its rational boundary components, with the Satake topology (cf. $[\mathrm{BB}])$. The closure $D_{1}^{*}$ in $D^{*}$ of a rational boundary component $D_{1}$ is again the Satake partial compactification of $D_{1}$.

If $D_{1} \subset D^{*}$ is a rational boundary component of $D$, its normalizer $P$ is the group of real points of a rationally defined maximal proper parabolic subgroup $\mathbf{P} \subset \mathbf{G}$. Let $\mathcal{U}_{P}$ denote 
the unipotent radical of $P$ and let $L(P)=P / \mathcal{U}_{P}$ be the Levi quotient with projection $\nu_{P}: P \rightarrow L(P)$. It is well known that $L(P)$ is an almost direct product (commuting product with finite intersection) of two subgroups, $L(P)=G_{h} G_{\ell}$ where the Riemannian symmetric space associated to $G_{h}$ is Hermitian, and the Riemannian symmetric space associated to $G_{\ell}$ is a self adjoint homogeneous cone. (We assume the possible compact factors in $L(P)$ have been distributed among $G_{h}$ and $G_{\ell}$ so that each is defined over $\mathbb{Q}$. The factor $G_{h}$ may be trivial. The $\mathbb{Q}$-split component $\mathbf{S}_{\mathbf{P}}(\mathbb{R})$ of the center of $L_{P}$ is contained in the $G_{\ell}$ factor.) The choice of basepoint $x_{0} \in D$ determines a unique $\theta$-stable lift $L_{P}\left(x_{0}\right) \subset P$ of the Levi quotient [BoS] (which, from now on, we shall use without mention), as well as basepoints $x_{1} \in D_{1}$ in the boundary component $D_{1}$ such that $\operatorname{Stab}_{G_{h}}\left(x_{1}\right)=K \cap G_{h}$. We obtain a decomposition

$$
P=\mathcal{U}_{P} G_{h} G_{\ell}
$$

The group $P$ acts on the boundary component $D_{1}$ through the projection $\nu_{h}: P \rightarrow L(P) \rightarrow$ $G_{h} /\left(G_{h} \cap G_{\ell}\right)$ which also determines a diffeomorphism $G_{h} / K_{h} \cong D_{1}$ (where $\left.K_{h}=K \cap G_{h}\right)$. This projection $\nu_{h}$ also gives rise to a $P$-equivariant canonical projection

$$
\pi: D \rightarrow D_{1}
$$

by $\pi\left(u g_{h} g_{\ell} K_{P}\right)=g_{h} K_{h}$.

The "linear part" $G_{\ell}$ is reductive and contains the 1-dimensional $\mathbb{Q}$-split torus $\mathbf{S}_{\mathbf{P}}(\mathbb{R})$ in the center of the Levi quotient. If $\mathfrak{z} \subset \mathfrak{N}_{P}$ denotes the center of the Lie algebra $\mathfrak{N}_{P}$ of the unipotent radical of $P$, then the adjoint action of $G_{\ell}$ on $\mathfrak{z}$ has a unique open orbit $C(P)$ which is a self adjoint homogeneous cone.

7.2. Let $\mathbf{P}_{\mathbf{0}} \subset \mathbf{G}$ be a fixed minimal rational parabolic subgroup and define the standard parabolic subgroups to be those which contain $\mathbf{P}_{\mathbf{0}}$. Let $\mathbf{S}_{\mathbf{0}} \subset L\left(\mathbf{P}_{\mathbf{0}}\right)$ be the greatest $\mathbb{Q}$-split torus in the center of $L\left(\mathbf{P}_{\mathbf{0}}\right)$ and let $\Phi=\Phi\left(\mathbf{S}_{\mathbf{0}}, G\right)$ be the (relative) roots of $G$ in $\mathbf{S}_{\mathbf{0}}$ with positive roots $\Phi^{+}$consisting of those roots which appear in the unipotent radical $\mathcal{U}_{P_{0}}$ and resulting simple roots $\Delta$. Each simple root $\alpha$ corresponds to a vertex in the (rational) Dynkin diagram for $\mathbf{G}$ and also to a maximal standard parabolic subgroup $\mathbf{P}$ such that $\mathbf{S}_{\mathbf{P}} \subset \operatorname{ker}(\beta)$ for each $\beta \in \Delta-\{\alpha\}$.

7.3. Two maximal parabolic subgroups. For simplicity, let us assume that $\mathbf{G}$ is (almost) simple over $\mathbb{Q}$. The (rational) Dynkin diagram for $\mathbf{G}$ is linear, of type BC, and determines a canonical ordering among the maximal standard rational parabolic subgroups with $\mathbf{P}_{\mathbf{1}} \prec \mathbf{P}_{\mathbf{2}}$ iff $D_{1} \prec D_{2}$ (meaning that $D_{1} \subset D_{2}^{*} \subset D^{*}$ ) where $D_{i}$ is the rational boundary component fixed by $P_{i}$. Write $P_{1}=\mathcal{U}_{1} G_{1 h} G_{1 \ell}$ and $P_{2}=\mathcal{U}_{2} G_{2 h} G_{2 \ell}$ as in (7.1.1). If $\mathbf{P}_{\mathbf{1}} \prec \mathbf{P}_{\mathbf{2}}$ then $G_{1 h} \subset G_{2 h}$ and $G_{1 \ell} \supset G_{2 \ell}$. Let $\mathbf{P}=\mathbf{P}_{\mathbf{1}} \cap \mathbf{P}_{\mathbf{2}}$. In Figure $4, \Delta-\left\{\alpha_{1}\right\}$ and $\Delta-\left\{\alpha_{2}\right\}$ denote the simple roots corresponding to $P_{1}$ and $P_{2}$ respectively. 


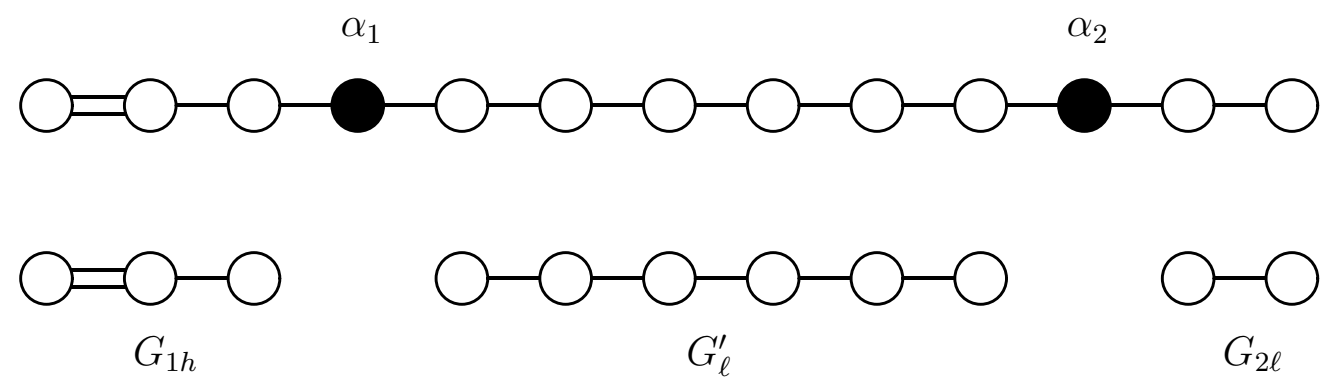

Figure 4. Dynkin diagrams for $G$ and $P$

Then we have a commutative diagram

$$
\begin{aligned}
& \mathcal{U}_{1} \subset \mathcal{U}_{P} \subset P \subset P_{1} \\
& \downarrow \downarrow \downarrow \nu_{1} \\
& \mathcal{U}_{\bar{P}} \subset \bar{P} \subset L\left(P_{1}\right)=G_{1 h} G_{1 \ell}
\end{aligned}
$$

where $\bar{P}=\nu_{1}(P) \subset L\left(P_{1}\right)$ is the image of $P$. Then $\bar{P}=G_{1 h} P_{\ell}$ where $P_{\ell} \subset G_{1 \ell}$ is a parabolic subgroup of $G_{1 \ell}$ whose Levi factor decomposes as a commuting, almost direct product $L\left(P_{\ell}\right)=G_{\ell}^{\prime} G_{2 \ell}$. Writing $\overline{\mathcal{U}}$ for the lift of $\mathcal{U}_{\bar{P}}=\mathcal{U}_{P_{\ell}}$ we conclude that $P$ has a decomposition

$$
P=\mathcal{U}_{1} G_{1 h}\left(\overline{\mathcal{U}} G_{\ell}^{\prime} G_{2 \ell}\right)=\mathcal{U}_{P} G_{1 h} G_{P \ell}
$$

with $\mathcal{U}_{P}=\mathcal{U}_{1} \overline{\mathcal{U}}, G_{P \ell}=G_{\ell}^{\prime} G_{2 \ell}$ and $P_{\ell}=\overline{\mathcal{U}} G_{\ell}^{\prime} G_{2 \ell} \subset G_{1 \ell}$. Similarly, we have a diagram

$$
\begin{gathered}
\mathcal{U}_{2} \subset \mathcal{U}_{P} \subset P \subset P_{2} \\
\downarrow \downarrow \downarrow \mathcal{L}^{\nu_{2}} \\
\mathcal{U}_{\overline{\bar{P}}} \subset \overline{\bar{P}} \subset L\left(P_{2}\right)=G_{2 h} G_{2 \ell}
\end{gathered}
$$

where $\overline{\bar{P}}=\nu_{2}(P) \subset L\left(P_{2}\right)$ is the image of $P$. Then $\overline{\bar{P}}=P_{h} G_{2 \ell}$ with $P_{h} \subset G_{2 h}$ a parabolic subgroup of $G_{2 h}$ whose Levi factor decomposes as a product $L\left(P_{h}\right)=G_{1 h} G_{\ell}^{\prime}$. Writing $\overline{\overline{\mathcal{U}}}$ for the canonical lift of $\mathcal{U}_{\bar{P}}=\mathcal{U}_{P_{h}}$ we obtain another decomposition,

$$
P=\mathcal{U}_{2}\left(\overline{\overline{\mathcal{U}}} G_{1 h} G_{\ell}^{\prime}\right) G_{2 \ell}=\mathcal{U}_{P} G_{1 h} G_{P \ell}
$$

with $P_{h}=\overline{\overline{\mathcal{U}}} G_{1 h} G_{\ell}^{\prime}$

Similarly, an arbitrary standard parabolic subgroup $Q$ may be expressed in a unique way as an intersection $Q=P_{1} \cap P_{2} \cap \ldots \cap P_{m}$ of maximal standard parabolic subgroups, with $\mathbf{P}_{\mathbf{1}} \prec \mathbf{P}_{\mathbf{2}} \prec \ldots \prec \mathbf{P}_{\mathbf{m}}$. In this case we write $Q^{b}=P_{1}$ and $Q^{\sharp}=P_{m}$. If $P_{1}=\mathcal{U}_{1} G_{1 h} G_{1 \ell}$ (with 
projection $\left.\nu_{1}: P_{1} \rightarrow G_{1 h} G_{1 \ell}\right)$ then the Levi factor $L(Q)$ decomposes as an almost direct product of $m+1$ factors

$$
L(Q)=G_{1 h}\left(G_{1}^{\prime} G_{2}^{\prime} \ldots G_{m-1}^{\prime} G_{m \ell}\right)=G_{1 h} G_{Q \ell}
$$

where $G_{1 h}$ is the hermitian part of $L\left(P_{1}\right)$, and $G_{Q \ell}$ consists of the remaining factors, including $G_{m \ell}$, the linear part of $L\left(P_{m}\right)$. Each factor in $G_{Q \ell}$ acts as an automorphism group of a certain symmetric cone in the boundary of the cone $C\left(P_{1}\right)$. The projection

$$
\bar{Q}=\nu_{1}(Q)=G_{1 h} \mathcal{U}_{P_{1} Q} G_{Q \ell}
$$

is parabolic in $L\left(P_{1}\right)$ with unipotent radical $\mathcal{U}_{P_{1} Q} \subset G_{1 \ell}$ which also has a lift depending on the choice of basepoint. In summary we obtain a decomposition

$$
Q=\mathcal{U}_{1} G_{1 h} \mathcal{U}_{P_{1} Q} G_{Q \ell}
$$

\section{CAYley transform}

8.1. As in $\S 7$, suppose that $\mathbf{G}$ is defined over $\mathbb{Q}$ and simple over $\mathbb{Q}$, that $G=\mathbf{G}(\mathbb{R})$, and that $K$ is a maximal compact subgroup of $G$ with $D=G / K$ Hermitian. The following proposition is the key technical tool behind our construction of a connection which is flat along the fibers of $\pi$. The proof follows from the existence of a "canonical automorphy factor for $P_{1}$ " as defined by M. Harris [Har2] [HZ1] (1.8.7). See also the survey in [Z3]. In this section we will approximately follow [Har2] and derive these results from known facts about the Cayley transform [WK], [Sat] Chapter III.

8.2. Proposition. Let $P_{1}=\mathcal{U}_{1} G_{1 h} G_{1 \ell}$ be a maximal rational parabolic subgroup of $\mathbf{G}$. Set $K_{1}=K \cap P_{1}=K_{1 h} K_{1 \ell}$. Let $\lambda: K \rightarrow G L(V)$ be a representation of $K$. Then the restriction $\lambda \mid K_{1}$ admits a natural extension $\lambda_{1}: K_{1 h} G_{1 \ell} \rightarrow G L(V)$.

8.3. Proof. The group $K$ is the set of real points of an algebraic group $\mathbf{K}$ defined over $\mathbb{R}$. As in [He] VIII $\S 7$, [Sat] II $\S 4$, [AMRT] III $\S 2$ when the Cartan decomposition $\mathfrak{g}=\mathfrak{k} \oplus \mathfrak{p}$ is complexified it gives rise to two abelian unipotent subgroups $P^{+}$and $P^{-}$of $\mathbf{G}(\mathbb{C})$ such that the complex structure on $\mathfrak{g}(\mathbb{C})$ acts with eigenvalue $\pm i$ on $\operatorname{Lie}\left(P^{ \pm}\right)$. The natural mapping $P^{+} \mathbf{K}(\mathbb{C}) P^{-} \rightarrow \mathbf{G}(\mathbb{C})$ is injective and its image contains $G=\mathbf{G}(\mathbb{R})$. Let $j: P^{+} \mathbf{K}(\mathbb{C}) P^{-} \rightarrow$ $\mathbf{K}(\mathbb{C})$ denote the projection to the middle factor. The group $P^{+}$is the unipotent radical of the maximal parabolic subgroup $P^{+} \mathbf{K}(\mathbb{C})$ and hence is normal in $P^{+} \mathbf{K}(\mathbb{C})$; similarly $P^{-}$is normal in $\mathbf{K}(\mathbb{C}) P^{-}$. It follows that, for all $h \in P^{+} \mathbf{K}(\mathbb{C})$, for all $h^{\prime} \in \mathbf{K}(\mathbb{C}) P^{-}$and for all $g \in P^{+} \mathbf{K}(\mathbb{C}) P^{-}$we have

$$
j\left(h g h^{\prime}\right)=j(h) j(g) j\left(h^{\prime}\right) .
$$

In particular, $j(g k)=j(g) k$ whenever $k \in \mathbf{K}(\mathbb{C})$, so by equation (5.6.1), $j$ determines a unique automorphy factor (the "usual" canonical automorphy factor) $J_{0}: G \times D \rightarrow \mathbf{K}(\mathbb{C})$ such that $J_{0}\left(g, x_{0}\right)=j(g)$ (for all $\left.g \in G\right)$.

Let $c_{1} \in \mathbf{G}(\mathbb{C})$ be the Cayley element [Sat],[WK], [BB], [Har2], [Z3] which corresponds to $\mathbf{P}_{\mathbf{1}}$. That is, $c_{1}$ is a lift to $\mathbf{G}(\mathbb{C})$ of the standard choice of Cayley element in $\operatorname{Ad}(\mathfrak{g})(\mathbb{C})$. 
We follow Satake's convention, rather than that of [WK] (which would associate $c_{1}^{-1}$ to $\mathbf{P}_{1}$.) The element $c_{1}$ satisfies the following properties, [Sat] (Chapt. III (7.8),(7.9),(2.4)), [Har2], [Z3]:

(1) $c_{1}, c_{1}^{-1} \in P^{+} \mathbf{K}(\mathbb{C}) P^{-}$and $c_{1} G \subset P^{+} \mathbf{K}(\mathbb{C}) P^{-}$;

(2) $c_{1}$ commutes with $G_{1 h}$;

(3) $c_{1} \mathcal{U}_{1} K_{1 h} G_{1 \ell} c_{1}^{-1} \subset P^{+} \mathbf{K}(\mathbb{C})$;

(4) $c_{1} K_{1 h} G_{1 \ell} c_{1}^{-1} \subset \mathbf{K}(\mathbb{C})$, and hence

(5) $j\left(c_{1} g\right)=j\left(c_{1} g c_{1}^{-1}\right) j\left(c_{1}\right)=c_{1} g c_{1}^{-1} j\left(c_{1}\right)$ for all $g \in K_{1 h} G_{1 \ell}$.

Harris then defines the canonical automorphy factor (for $\mathbf{P}_{\mathbf{1}}$ ) to be the automorphy factor $J_{1}: G \times D \rightarrow \mathbf{K}(\mathbb{C})$ which is determined by its values at the basepoint, $J_{1}\left(g, x_{0}\right)=$ $j\left(c_{1}\right)^{-1} j\left(c_{1} g\right)$. This is well defined because $j\left(c_{1}\right)^{-1} j\left(c_{1} g k\right)=j\left(c_{1}\right)^{-1} j\left(c_{1} g\right) k$ by (8.3.1), see (5.6.1). So we may define the canonical extension

$$
\lambda_{1}(g)=\lambda_{\mathbb{C}} J_{1}\left(g, x_{0}\right)=\lambda_{\mathbb{C}}\left(j\left(c_{1}\right)^{-1} j\left(c_{1} g\right)\right)
$$

for any $g \in K_{1 h} G_{1 \ell}$, where $\lambda_{\mathbb{C}}$ is the complexification of $\lambda$. Then $\lambda_{1}(k)=\lambda(k)$ for any $k \in K_{1}$. Moreover $\lambda_{1}$ is a homomorphism: if $k_{1 h} g_{1 \ell}$ and $k_{1 h}^{\prime} g_{1 \ell}^{\prime}$ are elements of $K_{1 h} G_{1 \ell}$ then

$$
\begin{aligned}
J_{1}\left(k_{1 h} g_{1 \ell} k_{1 h}^{\prime} g_{1 \ell}^{\prime}, x_{0}\right) & =j\left(c_{1}\right)^{-1}\left(c_{1} k_{1 h} g_{1 \ell} c_{1}^{-1} \cdot c_{1} k_{1 h}^{\prime} g_{1 \ell}^{\prime} c_{1}^{-1}\right) j\left(c_{1}\right) \\
& =j\left(c_{1}\right)^{-1} c_{1} k_{1 h} g_{1 \ell} c_{1}^{-1} j\left(c_{1}\right) \cdot j\left(c_{1}\right)^{-1} c_{1} k_{1 h}^{\prime} g_{1 \ell}^{\prime} c_{1}^{-1} j\left(c_{1}\right) \\
& =J_{1}\left(k_{1 h} g_{1 \ell}, x_{0}\right) J_{1}\left(k_{1 h}^{\prime} g_{1 \ell}^{\prime}, x_{0}\right) .
\end{aligned}
$$

Verification that $J_{1}\left(k_{1 h}^{-1} g_{1 \ell}^{-1}, x_{0}\right)=J_{1}\left(k_{1 h} g_{1 \ell}, x_{0}\right)^{-1}$ is similar. We remark, following [Har2] that modifying $c_{1}$ by any element $d \in P^{+} \mathbf{K}(\mathbb{C})$ will not affect the values of $J_{1}\left(k_{1 h} g_{1 \ell}, x_{0}\right)$.

Now suppose $\mathbf{P}_{\mathbf{1}} \prec \mathbf{P}_{\mathbf{2}}$ are rational maximal parabolic subgroups of $\mathbf{G}$ with $P_{i}=\mathcal{U}_{i} G_{i h} G_{i \ell}$ $(i=1,2)$ and, as in (7.3.4),

$$
P_{1} \cap P_{2}=\mathcal{U}_{2}\left(\overline{\overline{\mathcal{U}}} G_{1 h} G_{\ell}^{\prime}\right) G_{2 \ell} \text { with } P_{h}=\overline{\overline{\mathcal{U}}} G_{1 h} G_{\ell}^{\prime} \subset G_{2 h}
$$

8.4. Proposition. Let $\lambda_{i}: K_{i h} G_{i \ell} \rightarrow G L(V)$ be the canonical extensions of $\lambda \mid K_{i}=K \cap$ $P_{i}(i=1,2)$ and let $\lambda_{21}: K_{1 h} G_{\ell}^{\prime} \rightarrow G L(V)$ be the canonical extension of $\lambda \mid K_{2 h} \cap P_{h}$ corresponding to the canonical automorphy factor $J_{21}$ for $P_{h} \subset G_{2 h}$. Then

$$
\lambda_{1}\left(g_{2 \ell}\right)=\lambda_{2}\left(g_{2 \ell}\right) \text { and } \lambda_{1}\left(g_{\ell}^{\prime}\right)=\lambda_{21}\left(g_{\ell}^{\prime}\right)
$$

for all $g_{2 \ell} \in G_{2 \ell}$ and all $g_{\ell}^{\prime} \in G_{\ell}^{\prime}$.

8.5. Proof. Let $c_{1}, c_{2} \in \mathbf{G}(\mathbb{C})$ and $c_{21} \in \mathbf{G}_{\mathbf{2 h}}(\mathbb{C})$ be the Cayley elements for $P_{1}, P_{2} \subset G$ and $P_{h} \subset G_{2 h}$ respectively. By [Sat] Chapter III (9.5),

$$
c_{21}=c_{1} c_{2}^{-1}=c_{2}^{-1} c_{1} .
$$

The lift $G_{2 h} \subset G$ is stable under the Cartan involution on $G$ so the corresponding decomposition $P_{2 h}^{+} \mathbf{K}_{\mathbf{2 h}}(\mathbb{C}) P_{2 h}^{-}$coincides with $\left(\mathbf{G}_{\mathbf{2 h}}(\mathbb{C}) \cap P^{+}\right)\left(\mathbf{G}_{\mathbf{2 h}}(\mathbb{C}) \cap \mathbf{K}(\mathbb{C})\right)\left(\mathbf{G}_{\mathbf{2 h}}(\mathbb{C}) \cap P^{-}\right)$and in particular $j_{2 h}: G_{2 h} \rightarrow \mathbf{K}_{\mathbf{2 h}}(\mathbb{C})$ is the restriction of $j: G \rightarrow \mathbf{K}(\mathbb{C})$. Let $c_{21}=c_{21}^{+} c_{21}^{0} c_{21}^{-}$be 
the resulting decomposition. Then $c_{2}$ commutes with each of the factors $c_{21}^{*}$. It follows from (8.3.1) that

$$
j\left(c_{1}\right)=j\left(c_{21} c_{2}\right)=j\left(c_{21}^{+} c_{21}^{0} c_{2} c_{21}^{-}\right)=j\left(c_{21}^{0} c_{2}\right)=j\left(c_{21}\right) j\left(c_{2}\right)
$$

and similarly $j\left(c_{1}\right)=j\left(c_{2}\right) j\left(c_{21}\right)$. Since $g_{2 \ell} \in G_{2 \ell}$ also commutes with $c_{21} \in G_{2 h}(\mathbb{C})$ and with $j\left(c_{21}\right) \in K_{2 h}(\mathbb{C})$, we find

$$
\begin{aligned}
\lambda_{1}\left(g_{2 \ell}\right) \lambda_{2}\left(g_{2 \ell}\right)^{-1} & =\lambda_{\mathbb{C}}\left(j\left(c_{1}\right)^{-1} c_{1} g_{2 \ell} c_{1}^{-1} j\left(c_{1}\right) j\left(c_{2}\right)^{-1} c_{2} g_{2 \ell}^{-1} c_{2}^{-1} j\left(c_{2}\right)\right) \\
& =\lambda_{\mathbb{C}}\left(j\left(c_{1}\right)^{-1} c_{1} g_{2 \ell} c_{1}^{-1} j\left(c_{21}\right) c_{2} g_{2 \ell}^{-1} c_{2}^{-1} j\left(c_{2}\right)\right) \\
& =\lambda_{\mathbb{C}}\left(j\left(c_{1}\right)^{-1} c_{1} g_{2 \ell} c_{1}^{-1} c_{2} g_{2 \ell}^{-1} c_{2}^{-1} j\left(c_{21}\right) j\left(c_{2}\right)\right) \\
& =\lambda_{\mathbb{C}}\left(j\left(c_{1}\right)^{-1} c_{1} g_{2 \ell} c_{21}^{-1} g_{2 \ell}^{-1} c_{2}^{-1} j\left(c_{1}\right)\right) \\
& =\lambda_{\mathbb{C}}\left(j\left(c_{1}\right)^{-1} c_{1} c_{21}^{-1} c_{2}^{-1} j\left(c_{1}\right)\right)=1 .
\end{aligned}
$$

Similarly if $g_{\ell}^{\prime} \in G_{\ell}^{\prime}$ then using (4) above,

$$
\begin{aligned}
\lambda_{21}\left(g_{\ell}^{\prime}\right) & =\lambda_{\mathbb{C}}\left(j\left(c_{21}\right)^{-1} j\left(c_{21} g_{\ell}^{\prime} c_{21}^{-1}\right) j\left(c_{21}\right)\right) \\
& =\lambda_{\mathbb{C}}\left(j\left(c_{21}\right)^{-1} j\left(c_{2}^{-1} c_{1} g_{\ell}^{\prime} c_{1}^{-1} c_{2}\right) j\left(c_{21}\right)\right) \\
& =\lambda_{\mathbb{C}}\left(j\left(c_{21}\right)^{-1} j\left(c_{2}\right)^{-1} j\left(c_{1} g_{\ell}^{\prime} c_{1}^{-1}\right) j\left(c_{2}\right) j\left(c_{21}\right)\right) \\
& =\lambda_{\mathbb{C}}\left(j\left(c_{1}\right)^{-1} j\left(c_{1} g_{\ell}^{\prime} c_{1}^{-1}\right) j\left(c_{1}\right)\right)=\lambda_{1}\left(g_{\ell}^{\prime}\right) .
\end{aligned}
$$

\section{BAILY-Borel SATAKE COMPACTIFICATION}

9.1. As in $\S 7$, suppose that $\mathbf{G}$ is defined over $\mathbb{Q}$ and simple over $\mathbb{Q}$, that $G=\mathbf{G}(\mathbb{R})$, and that $K$ is a maximal compact subgroup of $G$ with $D=G / K$ Hermitian. Let $D^{*}$ be the Satake partial compactification of $D$, consisting of $D$ together with its rational boundary components $D_{P}$, one for each (proper) maximal rational parabolic subgroup $\mathbf{P} \subset \mathbf{G}$; with the Satake topology $[\mathrm{BB}]$. The action of $\mathbf{G}(\mathbb{Q})$ on $D$ extends continuously to an action of $\mathbf{G}(\mathbb{Q})$ on $D^{*}$. Let $\Gamma \subset \mathbf{G}(\mathbb{Q})$ be a neat arithmetic subgroup and let $q: D^{*} \rightarrow \bar{X}=\Gamma \backslash D^{*}$ denote the quotient mapping. Then $\bar{X}$ is the Baily-Borel compactification of $X$ and it admits the structure of a complex projective algebraic variety with a canonical stratification with a single stratum for every $\Gamma$-conjugacy class of rational boundary components as follows. Let $D_{1} \subset D^{*}$ be a rational boundary component with normalizing maximal parabolic subgroup $P=\mathcal{U}_{P} G_{h} G_{\ell}$. Let $\nu_{h}: P \rightarrow G_{h}^{\prime}=G_{h} /\left(G_{h} \cap G_{\ell}\right)$ and let $\nu_{\ell}: P \rightarrow G_{\ell}^{\prime}=G_{\ell} /\left(G_{h} \cap G_{\ell}\right)$. The closure $D_{1}^{*}$ of $D_{1}$ in $D^{*}$ is the Satake partial compactification of $D_{1}$. The group $P$ acts on $D_{1}$ through its projection to $G_{h}^{\prime}$ and the group $\Gamma_{P}=\Gamma \cap P$ acts on $D_{1}^{*}$ through its projection $\Gamma_{h}=\nu_{h}\left(\Gamma_{P}\right)$ to $G_{h}^{\prime}$. Then $X_{1}=\Gamma_{P} \backslash D_{1}^{*}=\Gamma_{h} \backslash D_{1}^{*}$ is a stratum of $\bar{X}$. Its closure $\bar{X}_{1}=\Gamma_{h} \backslash D_{1}^{*}$ in $\bar{X}$ is the Baily-Borel compactification of $X_{1}$. The stratum $X_{1}$ is also the image of the (infinitely many) rational boundary components $D_{1}^{\prime}$ which are $\Gamma$-conjugate to $D_{1}$. 
9.2. Let $D_{1} \subset D^{*}$ be a rational boundary component which projects to $X_{1}$. We will say that a neighborhood $\tilde{U} \subset D^{*}$ is a $\Gamma$-parabolic neighborhood of $D_{1}$ if the following holds: if $x_{1}, x_{2} \in \tilde{U}$ and $\gamma \in \Gamma$ satisfy $x_{2}=\gamma x_{1}$ then $\gamma \in \Gamma \cap P$. If $X_{1} \subset \bar{X}$ is a stratum in the Baily-Borel compactification of $X$, we say that a neighborhood $U \subset \bar{X}$ of $X_{1}$ is parabolic if for some (and hence for any) boundary component $D_{1} \subset D^{*}$ with $q\left(D_{1}\right)=X_{1}$, there is a $\Gamma$-parabolic neighborhood $\tilde{U} \subset D^{*}$ of $D_{1}$ such that $U=q(\tilde{U})$. This means that the covering $\Gamma_{P} \backslash D^{*} \rightarrow \Gamma \backslash D^{*}$ is one to one on $\tilde{U}$, and we have a commutative diagram

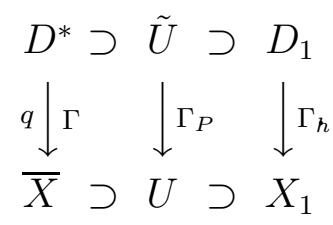

9.3. Lemma. Each stratum $X_{1} \subset \bar{X}$ has a fundamental system of neighborhoods, each of which is parabolic.

9.4. Proof. (We are grateful to L. Saper for providing us with the following argument.) Let $D^{B S}$ be the Borel-Serre partial compactification of $D$ together with its "Satake" topology $[\mathrm{BoS}]$. It is a manifold with corners, having one corner $e(P)$ for each rational parabolic subgroup $\mathbf{P}$. According to [Z2] the identity mapping $D \rightarrow D$ has a unique continuous extension $\mathfrak{w}: D^{B S} \rightarrow D^{*}$, and it is surjective. If $\mathbf{P}$ is standard then $\mathfrak{w}(e(P))=D_{P^{b}}$. Let $D^{\dagger}$ denote the quotient topology on the underlying set $\left|D^{*}\right|$ which is induced by $\mathfrak{w}$. Then $D^{\dagger} \rightarrow$ $D^{*}$ is a continuous bijection and the quotient mapping $\Gamma \backslash D^{\dagger} \rightarrow \Gamma \backslash D^{*}$ is a homeomorphism. In [Sa1] Theorem 8.1, Saper constructs a basis of $\Gamma$-parabolic neighborhoods $U_{P}$ of each corner $e(P)$ in $D^{B S}$. If $\mathbf{P}$ is maximal then $\mathfrak{w}\left(U_{P}\right)$ is open in $D^{*}$ as may be shown by verifying the condition at the bottom of page 264 in [AMRT].

We remark that the image of $U_{P}$ is $\Gamma$-parabolic and is open in $D^{\dagger}$ by construction, and that the topology $D^{\dagger}$ may be substituted for the Satake topology $D^{*}$ throughout this paper.

9.5. Fix a standard rational boundary component $D_{1} \subset D^{*}$ normalized by a standard maximal rational parabolic subgroup $P_{1}$. In the Satake topology (or in the topology $D^{\dagger}$ ) there is a natural neighborhood $T\left(D_{1}\right)=\bigcup\left\{D_{2} \mid D_{1} \prec D_{2} \prec D\right\}$ consisting of the union of all rational boundary components (including $D$, the nonproper boundary component) whose closures contain $D_{1}$. The projection $\pi: D \rightarrow D_{1}$ (7.1.2) has a unique continuous extension $T\left(D_{1}\right) \rightarrow D_{1}$ to this neighborhood. Its restriction to each intermediate boundary component $D_{2}$ coincides with the canonical projection $D_{2} \rightarrow D_{1}$ which is obtained by considering $D_{2}$ to be the symmetric space corresponding to the Hermitian part $G_{2 h}$ of the Levi factor of $P_{2}$ and by considering $D_{1} \subset D_{2}^{*}$ to be the rational boundary component preserved by the parabolic subgroup $P_{h} \subset G_{2 h}$ (notation as in $\S 7.3$ ). It follows that $\pi_{1}(x)=\pi_{1} \pi_{2}(x)$ for all $x \in T\left(D_{1}\right) \cap T\left(D_{2}\right)$. (The above union can be quite large: if $D_{20}$ is a standard boundary component normalized by a standard parabolic subgroup $\mathbf{P}_{\mathbf{2}} \succ \mathbf{P}_{\mathbf{1}}$ then the boundary 
components $D_{2} \subset T\left(D_{1}\right)$ which are conjugate to the standard one $D_{20}$ are in one to one correspondence with elements of $\mathbf{P}_{\mathbf{1}}(\mathbb{Q}) /\left(\mathbf{P}_{\mathbf{1}}(\mathbb{Q}) \cap \mathbf{P}_{\mathbf{2}}(\mathbb{Q})\right)$ cf. (7.3.2).)

9.6. Lemma. For $\epsilon_{0}>0$ sufficiently small, the Baily-Borel compactification $\bar{X}$ admits an $\epsilon_{0}$-system of control data $\left\{T_{Y}\left(\epsilon_{0}\right), \pi_{Y}, \rho_{Y}\right\}$ such that for each stratum $Y \subset \partial \bar{X}$ and for any choice of boundary component $D_{1} \subset D^{*}$ with $q\left(D_{1}\right)=Y$ we have

(1) The neighborhood $T_{Y}\left(\epsilon_{0}\right) \subset \bar{X}$ is a parabolic neighborhood of $Y$; it is the image, say, of some $\Gamma$-parabolic neighborhood $\tilde{U}\left(\epsilon_{0}\right) \subset D^{*}$ of $D_{1}$, and

(2) $\pi_{Y}(q(x))=q(\pi(x))$ for all $x \in \tilde{U}\left(\epsilon_{0}\right)$ (where $\pi: D \rightarrow D_{1}$ is the canonical projection).

9.7. Proof. For any $g \in P$ and $x \in D$ we have $\pi(g x)=\nu_{h}(g) \pi(x) \in D_{1}$. It follows that the projection function $\pi$ passes to the quotient $\Gamma_{P} \backslash D^{*}$, where it may be restricted to a parabolic neighborhood $U$ of $Y=q\left(D_{1}\right)$; write $\pi_{Y}: U \rightarrow Y$ for the result. If $P$ and $P^{\prime}$ are $\Gamma$ conjugate maximal rational parabolic subgroups corresponding to conjugate boundary components $D_{1}$ and $D_{1}^{\prime}$ then the projections $T\left(D_{1}\right) \rightarrow D_{1}$ and $T\left(D_{1}^{\prime}\right) \rightarrow D_{1}^{\prime}$ are compatible with conjugation, which shows that the resulting projection $\pi_{Y}: U \rightarrow Y$ is independent of the choice of lift $D_{1} \subset D^{*}$ of the stratum $Y \subset \bar{X}$. The tubular neighborhood $T_{Y}\left(\epsilon_{0}\right)$ may be chosen inside $U$. The compatibility between these projections follows from (9.5). As mentioned in $\S 2.3$, by further shrinking the tubular neighborhoods if necessary, control data may be found for which the tubular projections agree with these $\pi_{Y}$. (In fact it is possible to construct particular tubular distance functions $\rho_{Y}$ by smoothing Saper's piecewise analytic distance functions $\tilde{a}_{R}$ of [Sa1] thm. 8.1. See also [GM4] §4.2.)

\section{Parabolically Induced Connection}

10.1. As in $\S 7,9$ we suppose that $\mathbf{G}$ is semisimple, defined over $\mathbb{Q}$ and simple over $\mathbb{Q}$; that $G=\mathbf{G}(\mathbb{R})$, and $K \subset G$ is a maximal compact subgroup with $D=G / K$ Hermitian symmetric. Fix $\Gamma \subset \mathbf{G}(\mathbb{Q})$ a neat arithmetic subgroup. Let $\lambda: K \rightarrow G L(V)$ be a representation of $K$ on some complex vector space $V$ and denote by $E=G \times{ }_{K} V$ the associated homogeneous vector bundle on $D$.

Let $D_{1}$ be a rational boundary component of $D$ with canonical projection $\pi: D \rightarrow D_{1}$. Let $P$ be the maximal parabolic subgroup of $G$ which preserves $D_{1}$. Write $P=\mathcal{U} G_{h} G_{\ell}$ as in $\S 7.1$ and let $K_{h}=K \cap G_{h}$ and $K_{\ell}=K \cap G_{\ell}$ be the corresponding maximal compact subgroups. Let $\mathfrak{g}_{h}=\mathfrak{k}_{h} \oplus \mathfrak{p}_{h}$ and $\mathfrak{g}_{\ell}=\mathfrak{k}_{\ell} \oplus \mathfrak{p}_{\ell}$ denote the corresponding Cartan decompositions.

The restriction of $\lambda$ to $K_{h}$ determines a homogeneous vector bundle $E_{1}=G_{h} \times_{K_{h}} V$ over $D_{1}$. By Proposition 8.2 the representation $\lambda \mid K_{h} K_{\ell}$ admits an extension to a representation $\lambda_{1}: K_{h} G_{\ell} \rightarrow G L(V)$. This extension determines an action of $P$ on $E_{1}$ which is given by

$$
u g_{h} g_{\ell \cdot} \cdot\left[g_{h}^{\prime}, v\right]=\left[g_{h} g_{h}^{\prime}, \lambda_{1}\left(g_{\ell}\right) v\right] .
$$

We obtain a vector bundle mapping (which covers $\pi$ ),

$$
\tilde{\Phi}: E=P \times_{K_{P}} V \rightarrow G_{h} \times_{K_{h}} V=E_{1}
$$


by $\tilde{\Phi}\left(\left[u g_{h} g_{\ell}, v\right]\right)=\left[g_{h}, \lambda_{1}\left(g_{\ell}\right) v\right]$. Then $\tilde{\Phi}$ induces an isomorphism,

$$
\Phi: E \cong \pi^{*}\left(E_{1}\right) ; \quad[g, v] \mapsto\left(g K_{P}, \tilde{\Phi}([g, v])\right) \in D \times E_{1}
$$

of $P$-homogeneous vector bundles (where $g \in P$ and $v \in V$ ).

10.2. Definition. Let $\nabla_{1}=d+\omega_{1}$ be a connection on $E_{1}$. The parabolically induced connection $\nabla=d+\omega$ on $E$ is defined to be the pullback $\nabla=\Phi^{*}\left(\nabla_{1}\right)$ of $\nabla_{1}$ under the isomorphism $\Phi$. It is the unique connection whose covariant derivative (5.2.3) satisfies

$$
\nabla_{v}\left(\Phi^{*}(s)\right)=\Phi^{*}\left(\left(\nabla_{1}\right)_{\pi_{*} v} s\right)
$$

for any section $s$ of $E_{1}$ and for any tangent vector $v \in T_{x} D$.

10.3. Proposition. Suppose $\nabla_{1}=d+\omega_{1}$ is a connection on $E_{1}=G_{h} \times_{K_{h}} V$. Let $\nabla=d+\omega$ denote the parabolically induced connection on $E=P \times_{K_{P}} V$. Then

$$
\omega\left(L_{g *}\left(\dot{u}+\dot{g}_{h}+\dot{g}_{\ell}\right)\right)=\lambda_{1}^{\prime}\left(\dot{g}_{\ell}\right)+A d\left(\lambda_{1}\left(g_{\ell}^{-1}\right)\right)\left(\omega_{1}\left(L_{g_{h} *}\left(\dot{g}_{h}\right)\right)\right)
$$

for any $g=u g_{h} g_{\ell} \in P$ and any $\dot{u}+\dot{g}_{h}+\dot{g}_{\ell} \in \operatorname{Lie}\left(\mathcal{U}_{P}\right) \oplus \mathfrak{g}_{h} \oplus \mathfrak{g}_{\ell}$.

10.4. Proof. Let $J_{1}: G_{h} \times D_{1} \rightarrow G L(V)$ be an automorphy factor for $E_{1}$, corresponding to a trivialization $E_{1} \cong D_{1} \times V$. Composing this with the isomorphism $\Phi: E \rightarrow \pi^{*}\left(E_{1}\right)$ determines an automorphy factor $J: P \times D \rightarrow G L(V)$ with

$$
J\left(u g_{h} g_{\ell}, x_{0}\right)=J_{1}\left(g_{h}, x_{1}\right) \lambda_{1}\left(g_{\ell}\right)
$$

where $x_{1}=\pi\left(x_{0}\right) \in D_{1}$ denotes the basepoint in $D_{1}$. To simplify notation we will write $j(g)$ and $j_{1}\left(g_{h}\right)$ rather than $J\left(g, x_{0}\right)$ and $J_{1}\left(g_{h}, x_{1}\right)$.

By (5.8.1), the connection $\nabla_{1}$ in $E_{1}$ determines a connection $\nabla^{J_{1}}=d+\eta_{1}$ in the $J_{1^{-}}$ trivialization $E_{1} \cong D_{1} \times V$ with

$$
\eta_{1}\left(q_{1 *}\left(X_{h}\right)\right)=j_{1}\left(g_{h}\right) \omega_{1}\left(X_{h}\right) j_{1}\left(g_{h}\right)^{-1}-d_{X_{h}}\left(j_{1}(g)\right) \circ j_{1}(g)^{-1}
$$

for any $g_{h} \in G_{h}$ and any $X_{h} \in T_{g_{h}} G_{h}$, where $q_{1}: G_{h} \rightarrow D_{1}$ denotes the projection.

The parabolically induced connection $\nabla$ in $E$ determines a connection $\nabla^{J}=d+\eta$ in the $J$-trivialization $E \cong D \times V$ with

$$
\eta\left(q_{*} X\right)=j(g) \omega(X) j(g)^{-1}-d_{X}(j(g)) \circ j(g)^{-1} .
$$

By (10.2.1) and (5.2.3) the connection forms $\eta$ and $\eta_{1}$ are related by $\eta\left(q_{*}(X)\right)=\eta_{1}\left(\pi_{*} q_{*}(X)\right)$ for any $X \in T_{g} G$. Take $X=L_{g *}\left(\dot{u}+\dot{g}_{h}+\dot{g}_{\ell}\right) \in T_{g} G$ and let $X_{h}=L_{g_{h} *}\left(\dot{g}_{h}\right) \in T_{g_{h}} G_{h}$ denote its projection to $G_{h}$. Then we have

$$
\begin{aligned}
j_{1}\left(g_{h}\right) \omega_{1}\left(X_{h}\right) j_{1}\left(g_{h}\right)^{-1}= & j_{1}\left(g_{h}\right) \lambda_{1}\left(g_{\ell}\right) \omega(X) \lambda_{1}\left(g_{\ell}\right)^{-1} j_{1}\left(g_{h}\right)^{-1} \\
& -j_{1}\left(g_{h}\right) d_{X}\left(\lambda_{1}\left(g_{\ell}\right)\right) \lambda_{1}\left(g_{\ell}\right)^{-1} j_{1}\left(g_{h}\right)^{-1}
\end{aligned}
$$

or, using (5.1.1)

$$
\omega_{1}\left(L_{g_{h} *}\left(\dot{g}_{h}\right)\right)=\lambda_{1}\left(g_{\ell}\right) \omega(X) \lambda_{1}\left(g_{\ell}^{-1}\right)-\lambda_{1}\left(g_{\ell}\right) \lambda_{1}^{\prime}\left(\dot{g}_{\ell}\right) \lambda_{1}\left(g_{\ell}^{-1}\right)
$$


10.5. Corollary. Suppose $\omega_{1} \in \mathcal{A}^{1}\left(G_{h}, \operatorname{End}(V)\right)$ commutes with the adjoint action of $\lambda_{1}$ : $G_{\ell} \rightarrow G L(V)$. Then the curvature form $\Omega$ of the parabolically induced connection $\nabla=$ $\Phi^{*}\left(\nabla_{1}\right)=d+\omega$ is controlled,

$$
\Omega=\pi^{*}\left(\Omega_{1}\right)
$$

where $\Omega_{1} \in \mathcal{A}_{\text {bas }}^{2}\left(G_{h}\right.$, End $\left.(V)\right)$ is the curvature form of $\nabla_{1}=d+\omega_{1}$.

10.6. Proof. Let us compute $\Omega(X, Y)$ where $X=L_{g *}(\dot{x})$ and $Y=L_{g *}(\dot{y})$, where $\dot{x}, \dot{y} \in$ $\operatorname{Lie}(P)$ and where $g=u g_{h} g_{\ell} \in P$. Set $\dot{x}=\dot{u}_{X}+\dot{g}_{X h}+\dot{g}_{X \ell} \in \operatorname{Lie}\left(\mathcal{U}_{P}\right) \oplus \mathfrak{g}_{h} \oplus \mathfrak{g}_{\ell}$ (and similarly for $\dot{y})$. By (10.3.1),

$$
\omega(X)=\omega_{1}\left(L_{g_{h} *}\left(\dot{g}_{X h}\right)\right)+\lambda_{1}^{\prime}\left(\dot{g}_{X \ell}\right)
$$

and similarly for $\omega(Y)$. Set $X_{h}=L_{g_{h} *}\left(\dot{g}_{X h}\right)$ and $Y_{h}=L_{g_{h} *}\left(\dot{g}_{Y h}\right)$. Using the structure equation (5.2.4) and the fact that $\operatorname{Lie}\left(\mathcal{U}_{P}\right)$ is an ideal in $\operatorname{Lie}(P)$ gives

$$
\begin{aligned}
\Omega(X, Y)= & X(\omega(Y))-Y(\omega(X))-\omega([X, Y])+[\omega(X), \omega(Y)] \\
= & X\left(\omega_{1}\left(L_{g_{h} *}\left(\dot{g}_{Y h}\right)\right)\right)+X\left(\lambda_{1}^{\prime}\left(\dot{g}_{Y \ell}\right)\right)-Y\left(\omega_{1}\left(L_{g_{h} *}\left(\dot{g}_{X h}\right)\right)\right)-Y\left(\lambda_{1}^{\prime}\left(\dot{g}_{X \ell}\right)\right) \\
& -\omega_{1}\left(L_{g_{h} *}\left(\left[\dot{g}_{X h}, \dot{g}_{Y h}\right]\right)\right)-\lambda_{1}^{\prime}\left(\left[\dot{g}_{X \ell}, \dot{g}_{Y \ell}\right]\right) \\
& +\left[\omega_{1}\left(L_{g_{h} *}\left(\dot{g}_{X h}\right)\right), \omega_{1}\left(L_{g_{h} *}\left(\dot{g}_{Y h}\right)\right)\right]+\left[\lambda_{1}^{\prime}\left(\dot{g}_{X \ell}\right), \lambda_{1}^{\prime}\left(\dot{g}_{Y \ell}\right)\right] \\
= & \left.\left.X_{h} \omega_{1}\left(L_{g_{h} *}\left(\dot{g}_{Y h}\right)\right)\right)-Y_{h} \omega_{1}\left(L_{g_{h} *}\left(\dot{g}_{X h}\right)\right)\right)-\omega_{1}\left(\left[X_{h}, Y_{h}\right]\right)+\left[\omega_{1}\left(X_{h}\right), \omega_{1}\left(Y_{h}\right)\right] \\
= & \Omega_{1}\left(X_{h}, Y_{h}\right) .
\end{aligned}
$$

10.7. If $\nabla_{1}=d+\omega_{1}$ is a connection on $E_{1}$ which is invariant under a subgroup $\Gamma_{h} \subset G_{h}$, then by (10.3.1) the induced connection $\Phi^{*}\left(\nabla_{1}\right)$ is invariant under the group $\nu_{h}^{*}\left(\Gamma_{h}\right) \subset P$ which is obtained by first projecting $\Gamma_{h}$ to $G_{h}^{\prime}=G_{h} /\left(G_{h} \cap G_{\ell}\right)$ then taking the pre-image under the projection $\nu_{h}: P \rightarrow G_{h}^{\prime}($ cf. $\S 7.1, \S 9.1$ ).

As in $\S 9$ let $\Gamma \subset G$ be a neat arithmetic subgroup with $X=\Gamma \backslash D$. Write $X_{1}=\Gamma_{h} \backslash D_{1}$ for the stratum in $\bar{X}=\Gamma \backslash D^{*}$ corresponding to the boundary component $D_{1}$. The homogeneous vector bundles $E \rightarrow D$ and $E_{1} \rightarrow D_{1}$ pass to automorphic vector bundles $E^{\prime} \rightarrow X$ and $E_{1}^{\prime} \rightarrow X_{1}$ respectively. Parabolic induction then passes to an operation on these vector bundles as follows. Suppose $\nabla_{1}^{\prime}$ is a connection on $E_{1}^{\prime}$. It pulls back to a $\Gamma_{h}$-invariant connection $\nabla_{1}$ on $E_{1} \rightarrow D_{1}$. The parabolically induced connection $\nabla=\Phi^{*}\left(\nabla_{1}\right)$ is invariant under $\Gamma_{P}=\Gamma \cap P \subset \nu_{h}^{*}\left(\Gamma_{h}\right)$ so it passes to a connection on $\Gamma_{P} \backslash E \rightarrow \Gamma_{P} \backslash X$. Since $T_{X_{1}}\left(\epsilon_{0}\right)$ is a parabolic neighborhood of $X_{1}$ in $\bar{X}$ this defines a connection $\nabla^{\prime}=\Phi_{X X_{1}}^{*}\left(\nabla_{1}^{\prime}\right)$ on the restriction $E^{\prime} \mid\left(X \cap T_{X_{1}}\left(\epsilon_{0}\right)\right)$.

This procedure may be applied to any pair of strata, say, $X_{2}<X_{1}$ of $\bar{X}$. Thus, if $\nabla_{2}^{\prime}$ is any connection on the automorphic vector bundle $E_{2}^{\prime} \rightarrow X_{2}$ defined by $\lambda$, then we obtain a parabolically induced connection

$$
\nabla_{1}^{\prime}=\Phi_{X_{1} X_{2}}^{*}\left(\nabla_{2}^{\prime}\right)
$$

on $E_{1}^{\prime} \mid\left(X_{1} \cap T_{X_{2}}\left(\epsilon_{0}\right)\right)$. However, if $X_{3}<X_{2}<X_{1}$ are strata of $\bar{X}$ and if $\nabla_{3}^{\prime}$ is a connection on $E_{3}^{\prime} \rightarrow X_{3}$ then the parabolically induced connection $\Phi_{X_{1} X_{3}}^{*}\left(\nabla_{3}^{\prime}\right)$ does not necessarily agree 
with the connection $\Phi_{X_{1} X_{2}}^{*} \Phi_{X_{2} X_{3}}^{*}\left(\nabla_{3}^{\prime}\right)$, even in the neighborhood $X_{1} \cap T_{X_{2}}\left(\epsilon_{0}\right) \cap T_{X_{3}}\left(\epsilon_{0}\right)$ where they are both defined, cf. Proposition 10.9 .

10.8. We will also need the following more technical result concerning parabolic induction for the proof of the main theorem. Suppose $P_{1} \prec P_{2} \prec \ldots \prec P_{r}$ are standard maximal parabolic subgroups with corresponding rational boundary components $D_{1} \prec D_{2} \prec \cdots \prec$ $D_{r}$. Let $Q=P_{1} \cap P_{2} \cap \ldots \cap P_{r}$. Then $Q^{b}=P_{1}=\mathcal{U}_{1} G_{1 h} G_{1 \ell}$ so by (7.3.6), $Q$ decomposes as $Q=\mathcal{U}_{Q} G_{1 h} G_{Q \ell}$.

Let $\lambda$ be a representation of $K$ with resulting homogeneous vector bundles $E_{i} \rightarrow D_{i}(1 \leq$ $i \leq r)$. By Proposition 8.2, $\lambda$ extends to a representation $\lambda_{1}$ of $K_{1 h} G_{1 \ell}$. For $1 \leq i<j \leq r$ let $\Phi_{j i}: E_{j} \rightarrow \pi_{j i}^{*} E_{i}$ denote the vector bundle isomorphism of (10.1.2) corresponding to the canonical projection $\pi_{j i}: D_{j} \rightarrow D_{i}$. Let $\Phi_{r}: E \rightarrow \pi_{r}^{*}\left(E_{r}\right)$ be the vector bundle isomorphism (10.1.2) corresponding to the canonical projection $\pi_{r}: D \rightarrow D_{r}$.

10.9. Proposition. Suppose $\nabla_{1}=d+\omega_{1}$ is a connection on $E_{1}$ and suppose that the connection form $\omega_{1} \in \mathcal{A}^{1}\left(G_{1 h}, \operatorname{End}(V)\right)$ commutes with the adjoint action of $\lambda_{1}\left(G_{1 \ell}\right)$. Let $\nabla=d+\omega$ denote the connection

$$
\nabla=\Phi_{r}^{*} \Phi_{r, r-1}^{*} \ldots \Phi_{21}^{*}\left(\nabla_{1}\right) .
$$

Then

$$
\omega\left(L_{g *}\left(\dot{u}_{Q}+\dot{g}_{1 h}+\dot{g}_{Q \ell}\right)\right)=\omega_{1}\left(L_{g_{1 h} *}\left(\dot{g}_{1 h}\right)\right)+\lambda_{1}^{\prime}\left(\dot{g}_{Q \ell}\right)
$$

for any $g=u_{Q} g_{1 h} g_{Q \ell} \in Q$ and any $\dot{u}_{Q}+\dot{g}_{1 h}+\dot{g}_{Q \ell} \in \operatorname{Lie}(Q)=\operatorname{Lie}\left(\mathcal{U}_{Q}\right)+\mathfrak{g}_{1 h}+\mathfrak{g}_{Q \ell}$. The curvature form $\Omega$ of the connection $\nabla$ satisfies

$$
\Omega=\pi_{1}^{*}\left(\Omega_{1}\right)
$$

where $\Omega_{1} \in \mathcal{A}_{\text {bas }}^{2}\left(G_{1 h}, \operatorname{End}(V)\right)$ is the curvature form of $\nabla_{1}$ and where $\pi_{1}: D \rightarrow D_{1}$ is the canonical projection.

10.10. Proof. First we determine the connection form of the connection

$$
\nabla_{r}=\Phi_{r, r-1}^{*} \Phi_{r-1, r-2}^{*} \ldots \Phi_{21}^{*}\left(\nabla_{1}\right)=d+\omega_{r}
$$

on the vector bundle $E_{r} \rightarrow D_{r}$. Set $P_{r}=\mathcal{U}_{r} G_{r h} G_{r \ell}$. The images under the projection $\nu_{r h}$ : $P_{r} \rightarrow G_{r h}$ of $Q \subset P_{1} \cap P_{r}$ are parabolic subgroups $\bar{Q} \subset \bar{P}_{1} \subset G_{r h}$. In fact, $\bar{Q}$ is the parabolic subgroup corresponding to the flag of rational boundary components $D_{1} \prec D_{2} \prec \cdots \prec D_{r-1}$ of $D_{r}$. By (7.3.4) there are compatible decompositions

$$
\begin{aligned}
& P_{r}=\mathcal{U}_{r} G_{r h} G_{r \ell} \\
& P_{1} \cap P_{r}=\mathcal{U}_{r}\left(\overline{\overline{\mathcal{U}}} G_{1 h} G_{\ell}^{\prime}\right) G_{r \ell} \quad \text { with } \bar{P}_{1}=\overline{\overline{\mathcal{U}}} G_{1 h} G_{\ell}^{\prime} \\
& Q=\mathcal{U}_{r} \overline{\overline{\mathcal{U}}} G_{1 h}\left(\mathcal{U}_{\bar{Q} \ell} G_{\bar{Q} \ell}\right) G_{r \ell} \text { with } \bar{Q}=\overline{\overline{\mathcal{U}}} \mathcal{U}_{\bar{Q} \ell} G_{1 h} G_{\bar{Q} \ell} .
\end{aligned}
$$


Corresponding to the maximal parabolic subgroup $\bar{P}_{1} \subset G_{r h}$ the representation $\lambda \mid K_{1 h} K_{\ell}^{\prime}$ has a canonical extension

$$
\lambda_{r 1}: K_{1 h} G_{\ell}^{\prime} \rightarrow \mathrm{GL}(V) .
$$

According to Proposition 8.4, $\lambda_{r 1}\left|G_{\ell}^{\prime}=\lambda_{1}\right| G_{\ell}^{\prime}$ which, by assumption, commutes with $\omega_{1}$. So by induction, for any $\bar{q}=u_{\bar{Q}} g_{1 h} g_{\bar{Q} \ell} \in \bar{Q}$ and for any $\dot{\bar{q}}=\dot{u}_{\bar{Q}}+\dot{g}_{1 h}+\dot{g}_{\bar{Q} \ell} \in \operatorname{Lie}(\bar{Q})$,

$$
\omega_{r}\left(L_{\bar{q} *}\left(\dot{u}_{\bar{Q}}+\dot{g}_{1 h}+\dot{g}_{\bar{Q} \ell}\right)\right)=\omega_{1}\left(L_{g_{1 h} *}\left(\dot{g}_{1 h}\right)\right)+\lambda_{1}^{\prime}\left(\dot{g}_{\bar{Q} \ell}\right) .
$$

Now we are in a position to compute $\nabla=\Phi_{r}^{*}\left(\nabla_{r}\right)=d+\omega$. By Proposition 10.3, for any $g=u_{r} g_{r h} g_{r \ell} \in P_{r}$, and any $\dot{g}=\dot{u}_{r}+\dot{g}_{r h}+\dot{g}_{r \ell} \in \operatorname{Lie}\left(P_{r}\right)$, we have:

$$
\omega\left(L_{g *}\left(\dot{u}_{r}+\dot{g}_{r h}+\dot{g}_{r \ell}\right)\right)=\operatorname{Ad}\left(\lambda_{r}\left(g_{r \ell}^{-1}\right)\right)\left(\omega_{r}\left(L_{g_{r h} *}\left(\dot{g}_{r h}\right)\right)\right)+\lambda_{r}^{\prime}\left(\dot{g}_{r \ell}\right) .
$$

By Proposition 8.4, $\lambda_{r}\left|G_{r \ell}=\lambda_{1}\right| G_{r \ell}$. Taking $g_{r h}=\bar{q}=u_{\bar{Q}} g_{1 h} g_{\bar{Q} \ell} \in \bar{Q}$ and

$$
\dot{g}_{r h}=\dot{\bar{q}}=\dot{u}_{\bar{Q}}+\dot{g}_{1 h}+\dot{g}_{\bar{Q} \ell} \in \operatorname{Lie}(\bar{Q})
$$

gives

$$
\omega\left(L_{g *}\left(\dot{u}_{r}+\dot{u}_{\bar{Q}}+\dot{g}_{1 h}+\dot{g}_{\bar{Q} \ell}+\dot{g}_{r \ell}\right)\right)=\operatorname{Ad}\left(\lambda_{1}\left(g_{r \ell}^{-1}\right)\right)\left(\omega_{1}\left(L_{g_{1 h} *}\left(\dot{g}_{1 h}\right)\right)+\lambda_{1}^{\prime}\left(\dot{g}_{\bar{Q} \ell}\right)\right)+\lambda_{1}^{\prime}\left(\dot{g}_{r \ell}\right) .
$$

By assumption, the adjoint action of $\lambda_{1}\left(G_{r \ell}\right)$ commutes with $\omega_{1} \in \mathcal{A}^{1}\left(G_{1 h}, \operatorname{End}(V)\right)$. Moreover $G_{r \ell}$ commutes with $G_{\bar{Q} \ell}$. Therefore the operator $\operatorname{Ad}\left(\lambda_{1}\left(g_{r \ell}^{-1}\right)\right)$ may be dropped in this equation which gives equation (10.9.1):

$$
\omega\left(L_{g *}\left(\dot{u}_{r}+\dot{u}_{\bar{Q}}+\dot{g}_{1 h}+\dot{g}_{\bar{Q} \ell}+\dot{g}_{r \ell}\right)\right)=\omega_{1}\left(L_{g_{1 h} *}\left(\dot{g}_{1 h}\right)\right)+\lambda_{1}^{\prime}\left(\dot{g}_{\bar{Q} \ell}+\dot{g}_{r \ell}\right) .
$$

Finally we compute the curvature $\Omega$ of the induced connection $\nabla$. Let $g=u_{Q} g_{1 h} g_{Q \ell} \in Q$, let $\dot{x}=\dot{u}_{x Q}+\dot{x}_{1 h}+\dot{x}_{Q \ell}$ and $\dot{y}=\dot{u}_{y Q}+\dot{y}_{1 h}+\dot{y}_{Q \ell} \in \operatorname{Lie}(Q)$. Then a calculation identical to that of $\$ 10.6$, using (10.9.1) in place of (10.6.1) gives:

$$
\Omega\left(L_{g *}(\dot{x}, \dot{y})\right)=\Omega_{1}\left(L_{g_{1 h} *}\left(\dot{x}_{1 h}, \dot{y}_{1 h}\right)\right)
$$

which implies that $\Omega=\pi_{1}^{*}\left(\Omega_{1}\right)$.

\section{The Patched Connection}

11.1. As $§ 7,9$, suppose that $D=G / K$ is a Hermitian symmetric space which is irreducible over $\mathbb{Q}$, and that $\Gamma \subset \mathbf{G}(\mathbb{Q})$ is a neat arithmetic group. Let $\bar{X}=\Gamma \backslash D^{*}$ denote the Baily Borel compactification of $X=\Gamma \backslash D$ with projection $q: D^{*} \rightarrow \bar{X}$. By lemma 9.6, for any sufficiently small $\epsilon_{0}>0$ there exists an $\epsilon_{0}$-system of control data $\left\{T_{Y}\left(\epsilon_{0}\right), \pi_{Y}, \rho_{Y}\right\}$ (which we now fix) on $\bar{X}$, so that $\pi_{Y}$ is obtained from the canonical projection $D \rightarrow D_{1}$ whenever $q\left(D_{1}\right)=Y$ and so that $T_{Y}(\epsilon)$ is a parabolic neighborhood of $Y$ in $\bar{X}$. Applying $\S 3.5$ to this system of control data yields a partition of unity on each stratum $Y$ of $\bar{X}$,

$$
B_{Y}^{\epsilon Y}(y)+\sum_{Z<Y} B_{Z}^{\epsilon Y}(y)=1
$$

for all $y \in Y$, where $\epsilon Y=\epsilon_{0} / 2^{\operatorname{dim} Y}$. 
A choice of representation $\lambda: K \rightarrow G L(V)$ on some complex vector space $V$ determines homogeneous vector bundles $E=G \times_{K} V$ on $D$ and $E_{1}=G_{h} \times_{K_{h}} V$ on $D_{1}$ which pass to automorphic vector bundles $E^{\prime}=\Gamma \backslash E$ on $X$ and $E_{Y}^{\prime} \rightarrow Y$ on $Y$. Here, $D_{1}$ is a rational boundary component (with $q\left(D_{1}\right)=Y$ ), normalized by some maximal parabolic subgroup $P=\mathcal{U}_{P} G_{h} G_{\ell}$; and $K_{h}=K \cap G_{h}$; cf. $\oint 7.1,9.1$. The Nomizu connections $\nabla_{D}^{\text {Nom }}$ (on $E)$ and $\nabla_{1}^{\text {Nom }}$ (on $E_{1}$ ) pass to connections $\nabla_{X}^{\text {Nom }}$ on $E^{\prime} \rightarrow X$ and $\nabla_{Y}^{\text {Nom }}$ on $E_{Y}^{\prime} \rightarrow Y$ respectively. We use an inductive procedure to define the patched connection $\nabla_{Y}^{\mathrm{p}}$ on the vector bundle $E_{Y}^{\prime} \rightarrow Y$, for any stratum $Y \leq X$ as follows. If $Y \subset \bar{X}$ is a minimal stratum set $\nabla_{Y}^{\mathrm{p}}=\nabla_{Y}^{\mathrm{Nom}}$. Now suppose that the patched connection $\nabla_{W}^{\mathrm{p}}$ has been constructed on every stratum $W<Y$.

11.2. Definition. The patched connection $\nabla_{Y}^{\mathrm{p}}$ on $E_{Y}^{\prime} \rightarrow Y$ is the connection

$$
\nabla_{Y}^{\mathrm{p}}=B_{Y}^{\epsilon Y} \nabla_{Y}^{\mathrm{Nom}}+\sum_{W<Y} B_{W}^{\epsilon Y} \Phi_{Y W}^{*}\left(\nabla_{W}^{\mathrm{p}}\right)
$$

(where the sum is taken over all strata $W<Y$ in the Baily Borel compactification of $X$ ).

11.3. Remarks. The idea behind this construction may be explained when there are two singular strata $Z<Y<X$. A simpler candidate for a connection on $X$ whose Chern forms might satisfy the control condition is

$$
\nabla_{X}^{\prime}=B_{Z}^{\epsilon X} \Phi_{X Z}^{*} \nabla_{Z}^{\mathrm{Nom}}+B_{Y}^{\epsilon X} \Phi_{X Y}^{*} \nabla_{Y}^{\mathrm{Nom}}+B_{X}^{\epsilon X} \nabla_{X}^{\mathrm{Nom}}
$$

In the region $T_{Y}(\epsilon X / 2)$ only the first two terms contribute to $\nabla_{X}^{\prime}$. Both connection $\Phi_{X Z}^{*} \nabla_{Z}^{\text {Nom }}$ and $\Phi_{X Y}^{*} \nabla_{Y}^{\text {Nom }}$ have curvature forms which are controlled with respect to $Y$. However the curvature form of (and even the Chern forms of) any affine combination of these fails to satisfy the control condition. (cf. Figure 3.4: this occurs in the region where $B_{Z}+B_{Y}=1$.) The remedy is to create a connection on $X$ for which no nontrivial affine combination of $\Phi_{X Z}^{*} \nabla_{Z}^{\mathrm{Nom}}$ and $\Phi_{X Y}^{*} \nabla_{Y}^{\mathrm{Nom}}$ ever occurs. Replacing $\nabla_{Y}^{\mathrm{Nom}}$ by $\nabla_{Y}^{\mathrm{p}}$ in (11.3.1) gives

$$
\nabla_{X}^{\mathrm{p}}=B_{Z}^{\epsilon X} \Phi_{X Z}^{*} \nabla_{Z}^{\mathrm{Nom}}+B_{Y}^{\epsilon X} B_{Z}^{\epsilon Y} \Phi_{X Y}^{*} \Phi_{Y Z}^{*} \nabla_{Z}^{\mathrm{Nom}}+B_{Y}^{\epsilon X} B_{Y}^{\epsilon Y} \Phi_{X Y}^{*} \nabla_{Y}^{\mathrm{Nom}}+B_{X}^{\epsilon X} \nabla_{X}^{\mathrm{Nom}}
$$

Within the region $T_{Y}(\epsilon X / 2)$ only the first three terms appear: the first term alone appears in $T_{Z}(\epsilon X / 2)$; the first and second terms appear in $T_{Z}(\epsilon X)-T_{Z}(\epsilon X / 2)$; the second term alone appears in $T_{Z}(\epsilon Y / 2)-T_{Z}(\epsilon X)$; the second and third terms appear in $T_{Z}(\epsilon Y)-T_{Z}(\epsilon Y / 2)$ and the third term alone appears outside $T_{Z}(\epsilon Y)$. In the region $T_{Z}(\epsilon Y)-T_{Z}(\epsilon Y / 2)$,

$$
\nabla_{X}^{\mathrm{p}}=\Phi_{X Y}^{*}\left(B_{Z}^{\epsilon Y} \Phi_{Y Z}^{*} \nabla_{Z}^{\mathrm{Nom}}+B_{Y}^{\epsilon Y} \nabla_{Y}^{\mathrm{Nom}}\right) .
$$

So $\nabla_{X}^{\mathrm{p}}$ is parabolically induced from a connection on $Y$, and by Corollary 10.5 its curvature form is controlled relative to $Y$. In the region $T_{Z}(\epsilon X)-T_{Z}(\epsilon X / 2)$,

$$
\nabla_{X}^{\mathrm{p}}=\left(B_{Z}^{\epsilon X} \Phi_{X Z}^{*}+B_{Y}^{\epsilon X} B_{Z}^{\epsilon Y} \Phi_{X Y}^{*} \Phi_{Y Z}^{*}\right)\left(\nabla_{Z}^{\mathrm{Nom}}\right) .
$$

In this region, the curvature form still does not satisfy the control condition however we show in $\S 12.10$ that the difference $\left(\Phi_{X Z}^{*}-\Phi_{X Y}^{*} \Phi_{Y Z}^{*}\right) \omega_{Z}^{\text {Nom }}$ is nilpotent and commutes with 
the curvature form. This turns out to be enough (Lemma 6.4) to imply that the Chern forms of $\nabla_{X}^{\mathrm{p}}$ are controlled with respect to $Y$.

11.4. Returning to the general case, suppose $\mathbf{Z}=Z(1)<Z(2)<\cdots<Z(r)$ is a chain of strata in $\bar{X}$. Write $\mathbf{Z} \leq Y$ if $Z(r)=Y$, that is, if the chain ends in $Y$. Write $Y \leq \mathbf{Z}$ if $Z(1)=Y$, that is, if the chain begins at $Y$. Suppose $\mathbf{Z}$ is such a chain of strata and suppose $x \in T_{Z(i)}\left(\epsilon_{0}\right)$ for $1 \leq i \leq r$. Denote by

$$
\begin{aligned}
\epsilon i & =\epsilon(Z(i))=\epsilon_{0} / 2^{\operatorname{dim} Z(i)} \\
B_{i}^{\epsilon} & =B_{Z(i)}^{\epsilon} \\
\Phi_{j i}^{*} & =\Phi_{Z(j) Z(i)}^{*} \text { for } j>i \\
\pi_{i}(x) & =\pi_{Z(i)}(x) .
\end{aligned}
$$

Define

$$
\begin{aligned}
B_{\mathbf{Z}}(x) & =B_{r-1}^{\epsilon r}\left(\pi_{r}(x)\right) \ldots B_{2}^{\epsilon 3}\left(\pi_{3}(x)\right) B_{1}^{\epsilon 2}\left(\pi_{2}(x)\right) \\
\Phi_{\mathbf{Z}}^{*} & =\Phi_{r, r-1}^{*} \ldots \Phi_{32}^{*} \Phi_{21}^{*} .
\end{aligned}
$$

If $\mathbf{Z}=\{Y\}$ consists of a single element, set $B_{\mathbf{Z}}(x)=1$ and $\Phi_{\mathbf{Z}}^{*}=\mathrm{Id}$.

11.5. Lemma. Let $Y$ be a stratum of $\bar{X}$. Then for all $x \in Y$,

$$
\sum_{\mathbf{Z} \leq Y} B_{\mathbf{Z}}(x) B_{Z(1)}^{\epsilon Z(1)}\left(\pi_{Z(1)}(x)\right)=1
$$

and the patched connection may be expressed as follows,

$$
\nabla_{Y}^{\mathrm{p}}(x)=\sum_{\mathbf{Z} \leq Y} B_{\mathbf{Z}}(x) \Phi_{\mathbf{Z}}^{*}\left(B_{Z(1)}^{\epsilon Z(1)}\left(\pi_{Z(1)}(x)\right) \nabla_{Z(1)}^{\mathrm{Nom}}\left(\pi_{Z(1)}(x)\right)\right)
$$

where (in both equations) the sum is over all chains of strata $\mathbf{Z} \leq Y$ ending in $Y$.

11.6. Remarks. One checks that, although the projection functions $x \mapsto \pi_{Z(i)}(x)$ are not everywhere defined, they occur in (11.5.1), (11.5.2) with coefficient 0 unless $x$ lies in the region of definition. Henceforth we will abbreviate (11.5.2) by

$$
\nabla_{Y}^{\mathrm{p}}=\sum_{\mathbf{Z} \leq Y} B_{\mathbf{Z}} \Phi_{\mathbf{Z}}^{*}\left(B_{Z(1)}^{\epsilon Z(1)} \nabla_{Z(1)}^{\mathrm{Nom}}\right)
$$

but we caution the reader about the possible ambiguity in this notation which arises from the fact that both $B_{Z(1)}^{\epsilon Z(1)}(x)$ and $B_{Z(1)}^{\epsilon Z(1)}\left(\pi_{Z(1)}(x)\right)$ are defined but do not necessarily coincide. 
11.7. Proof. We will prove (11.5.2) by induction; the proof of (11.5.1) is similar. Using induction, replace $\nabla_{W}^{\mathrm{p}}$ in (11.2.1) by the corresponding expression (11.5.2) to obtain

$$
\begin{aligned}
\nabla_{Y}^{\mathrm{p}}(x)= & B_{Y}^{\epsilon Y}(x) \nabla_{Y}^{\mathrm{Nom}}(x) \\
& +\sum_{W<Y} \sum_{\mathbf{Z}^{\prime} \leq W} B_{W}^{\epsilon Y}(x) B_{\mathbf{Z}^{\prime}}\left(\pi_{W}(x)\right) \Phi_{Y W}^{*} \Phi_{W \mathbf{Z}^{\prime}}^{*}\left(B_{Z^{\prime}(1)}^{\epsilon Z^{\prime}(1)} \nabla_{Z^{\prime}(1)}^{\mathrm{Nom}}\right) .
\end{aligned}
$$

In (11.5.2) the trivial chain $\mathbf{Z}=\{Y\}$ gives rise to a term $B_{Y}^{\epsilon Y}\left(\pi_{Y}(x)\right) \nabla_{Y}^{\mathrm{Nom}}$. Each nontrivial chain $\mathbf{Z}=\{Z(1)<\cdots<Z(r-1)<Z(r)=Y\}$ may be expressed uniquely as the concatenation of a 1-step chain $W=Z(r-1)<Y$ with a chain $\mathbf{Z}^{\prime}$ ending in $W$. Then $B_{\mathbf{Z}}(x)=B_{W}^{\epsilon Y}(x) B_{\mathbf{Z}^{\prime}}\left(\pi_{W}(x)\right)$ and $\Phi_{\mathbf{Z}}^{*}=\Phi_{Y W^{\prime}}^{*} \Phi_{\mathbf{Z}^{\prime}}^{*}$. When substituted into (11.5.2) these give (11.7.1), which completes the inductive step in the argument.

Definition 11.2 constructs a patched connection $\nabla_{Y}^{\mathrm{p}}$ on each of the automorphic vector bundles $E_{Y}^{\prime} \rightarrow Y$. The proof of the following theorem will appear in $\S 12$.

11.8. Theorem. The Chern forms $\left\{\sigma^{j}\left(\nabla_{Y}^{\mathrm{p}}\right)\right\}_{Y \leq X}$ of the patched connections $\nabla_{Y}^{\mathrm{p}}$ constitute a closed controlled differential form.

11.9. Corollary. For each $j$, the Chern form $\sigma^{j}\left(\nabla_{X}^{\mathrm{p}}\right) \in \mathcal{A}_{\pi}^{2 j}(\bar{X} ; \mathbb{C})$ of the patched connection determines a lift

$$
\bar{c}^{j}\left(E^{\prime}\right)=\left[\sigma^{j}\left(\nabla_{X}^{\mathrm{p}}\right)\right] \in H^{2 j}(\bar{X} ; \mathbb{C})
$$

of the Chern class $c^{j}\left(E^{\prime}\right) \in H^{2 j}(X ; \mathbb{C})$ which is independent of the choices that were made in its construction. For any stratum closure $i: \bar{Y} \hookrightarrow \bar{X}$ the restriction $i^{*} \bar{c}^{j}\left(E^{\prime}\right)$ is equal to the Chern class $\bar{c}^{j}\left(E_{Y}^{\prime}\right) \in H^{2 j}(\bar{Y} ; \mathbb{C})$ of the automorphic vector bundle $E_{Y}^{\prime} \rightarrow Y$.

11.10. Proof of Corollary 11.9. The restriction map $i^{*}: H^{2 j}(\bar{X}) \rightarrow H^{2 j}(X)$ associates to any controlled differential form $\omega \in \mathcal{A}_{\pi}^{2 j}(\bar{X})$ the cohomology class $\left[\omega_{X}\right]$ of the differential form $\omega_{X} \in \mathcal{A}^{2 j}(X)$ on the nonsingular part. Hence $i^{*}\left(\bar{c}^{j}\left(E^{\prime}\right)\right)=c^{j}\left(E^{\prime}\right) \in H^{2 j}(X ; \mathbb{C})$ since the latter is independent of the connection.

The patched connection $\nabla_{X}^{\mathrm{p}}$ depends on the choice of a pair (partition of unity, control data which is subordinate to the canonical projections $\left\{\pi_{Z}\right\}$ ) (see $\S 3$ and $\S 2$ ). It is tedious but standard to check that two such choices are connected by a smooth 1-parameter family of choices (partition of unity, control data subordinate to $\left\{\pi_{Z}\right\}$ ). The resulting patched connections $\nabla_{0}^{\mathrm{p}}$ and $\nabla_{1}^{\mathrm{p}}$ are therefore connected by a smooth 1-parameter family of patched connections $\nabla_{t}^{\mathrm{p}}$, each of whose Chern forms is a controlled differential form. So the usual argument (e.g. [KN] Chapt. XII lemma 5; [MiS]) produces a differential $2 j-1$ form $\Psi$ such that $\sigma^{j}\left(\nabla_{1}^{\mathrm{p}}\right)-\sigma^{j}\left(\nabla_{0}^{\mathrm{p}}\right)=d \Psi$. It is easy to see that $\Psi \in \mathcal{A}_{\pi}^{2 j-1}(\bar{X} ; \mathbb{C})$ is a controlled differential form. Consequently the controlled cohomology classes coincide: $\left[\sigma^{j}\left(\nabla_{1}^{\mathbf{p}}\right)\right]=\left[\sigma^{j}\left(\nabla_{0}^{\mathbf{p}}\right)\right] \in$ $H^{2 j}(\bar{X} ; \mathbb{C})$. The second statement follows from the analogous statement in Theorem 4.2. 
11.11. Remarks. Theorem 11.8 and Corollary 11.9 extend to the case that $\mathbf{G}$ is semisimple over $\mathbb{Q}$. The restriction map $H^{2 j}(\bar{X} ; \mathbb{C}) \rightarrow H^{2 j}(X ; \mathbb{C})$ factors as follows,

$$
H^{2 j}(\bar{X} ; \mathbb{C}) \rightarrow I H^{2 j}(\bar{X} ; \mathbb{C}) \rightarrow H_{2 n-2 j}(\bar{X} ; \mathbb{C}) \rightarrow H_{2 n-2 j}(\bar{X}, \partial \bar{X} ; \mathbb{C}) \cong H^{2 j}(X ; \mathbb{C})
$$

where $2 n=\operatorname{dim}_{\mathbb{R}}(X)$, and where $\partial \bar{X}$ denotes the singular set of the Baily-Borel compactification $\bar{X}$. The Chern class $c^{j}\left(E^{\prime}\right) \in H^{2 j}(X ; \mathbb{C})$ lives in the last group. For any toroidal resolution of singularities $\tau: \bar{X}_{\Sigma} \rightarrow \bar{X}$, the pushdown

$$
c_{n-j}\left(E^{\prime}\right)=\tau_{*}\left(c^{j}\left(\bar{E}_{\Sigma}^{\prime}\right) \cap\left[\bar{X}_{\Sigma}\right]\right) \in H_{2 n-2 j}(\bar{X} ; \mathbb{Z})
$$

of the Chern class of Mumford's canonical extension ([Mu1]) $\bar{E}_{\Sigma}^{\prime}$ of $E^{\prime}$ gives a canonical lift of $c^{j}\left(E^{\prime}\right)$ to the homology of the Baily-Borel compactification. In $\S 15$ (in the case of the tangent bundle) we identify this with the (homology) Chern class of the constructible function $\mathbf{1}_{X}$. In $[\mathrm{BBF}]$ it is shown that every algebraic homology class (including $c_{n-j}\left(E^{\prime}\right)$ ) admits a (non-canonical) lift to middle intersection homology with rational coefficients.

\section{Proof of Theorem 11.8}

12.1. Preliminaries. As in $\S 9.1$, let $q: D^{\star} \rightarrow \bar{X}$ denote the projection. If $\epsilon_{0}>0$ is sufficiently small, then for each stratum $Z$ of $\bar{X}$ the preimage

$$
q^{-1}\left(T_{Z}\left(\epsilon_{0}\right)\right)=\coprod_{q\left(D_{1}\right)=Z} U_{D_{1}}\left(\epsilon_{0}\right)
$$

is a disjoint union of $\Gamma$-parabolic neighborhoods $U_{D_{1}}\left(\epsilon_{0}\right)$ of those boundary components $D_{1}$ such that $q\left(D_{1}\right)=Z$. For such a boundary component define

$$
\chi_{D_{1}}(x)=\left\{\begin{array}{l}
1 \text { if } x \in U_{D_{1}}\left(\epsilon_{0}\right) \\
0 \text { otherwise }
\end{array}\right.
$$

to be the characteristic function of $U_{D_{1}}$.

Fix a stratum $Y$ and a choice $D_{2}$ of rational boundary component such that $q\left(D_{2}\right)=Y$. Denote by $P_{2}=\mathcal{U}_{2} G_{2 h} G_{2 \ell}$ the rational maximal parabolic subgroup of $G$ which normalizes $D_{2}$ and by $\nu_{h}: P_{2} \rightarrow G_{2 h}$ the projection as in $\S 9.1$. By Proposition 8.2 the representation $\lambda \mid K \cap P_{2}$ extends to a representation $\lambda_{2}$ of $K_{2 h} G_{2 \ell}$. Set $\Gamma_{h}=\nu_{h}\left(\Gamma \cap P_{2}\right)$.

The partition of unity $B_{Y}^{\epsilon Y}+\sum_{Z<Y} B_{Z}^{\epsilon Y}=1$ on $Y$ pulls back to a $\Gamma_{h}$-invariant locally finite partition of unity on $D_{2}$,

$$
B_{D_{2}}^{\epsilon Y}+\sum_{D_{1} \prec D_{2}} B_{D_{1}}^{\epsilon Y}=1
$$

where the sum is over all rational boundary components $D_{1} \prec D_{2}$, where $B_{D_{1}}^{\epsilon Y}=q^{*}\left(B_{Z}^{\epsilon Y}\right) \chi_{D_{1}}$ (and similarly for $B_{D_{2}}^{\epsilon Y}$, however $\chi_{D_{2}}=1$ on $D_{2}$ ). The patched connection $\nabla_{Y}^{\mathrm{p}}$ on the vector bundle $E_{Y}^{\prime} \rightarrow Y$ pulls back to a $\Gamma_{h}$-invariant connection $\nabla_{2}^{\mathrm{p}}=q^{*}\left(\nabla_{Y}^{\mathrm{p}}\right)$ on the homogeneous 
vector bundle $E_{2}=G_{2 h} \times_{K_{2 h}} V$. This connection may also be described as the affine locally finite combination

$$
\nabla_{2}^{\mathrm{p}}=B_{D_{2}}^{\epsilon Y} \nabla_{2}^{\mathrm{Nom}}+\sum_{D_{1} \prec D_{2}} B_{D_{1}}^{\epsilon Y} \Phi_{21}^{*}\left(\nabla_{1}^{\mathrm{p}}\right)
$$

where, for each rational boundary component $D_{1} \prec D_{2}$ the obvious notation holds: $\nabla_{1}^{\mathrm{p}}$ is the patched connection on $E_{1} \rightarrow D_{1}$ and $\Phi_{21}: E_{2} \rightarrow \pi_{21}^{*}\left(E_{1}\right)$ is the vector bundle isomorphism which is obtained from (10.1.3) upon replacing $G$ by $G_{2 h}$.

Denote by $\omega_{2}^{\mathrm{p}} \in \mathcal{A}^{1}\left(G_{2 h}\right.$, End $\left.(V)\right)$ the connection form of $\nabla_{2}^{\mathrm{p}}$. The curvature form $\Omega_{Y}^{\mathrm{p}} \in$ $\mathcal{A}^{2}\left(Y, \operatorname{End}\left(E_{Y}^{\prime}\right)\right)$ of $\nabla_{Y}^{\mathrm{p}}$ coincides with the curvature form $\Omega_{2}^{\mathrm{p}} \in \mathcal{A}_{\text {bas }}^{2}\left(G_{2 h}, \operatorname{End}(V)\right)$ of $\nabla_{2}^{\mathrm{p}}$ under the canonical isomorphism

$$
\mathcal{A}^{2}\left(Y, \operatorname{End}\left(E_{Y}^{\prime}\right)\right) \cong \mathcal{A}^{2}\left(D_{2}, \operatorname{End}\left(E_{2}\right)\right)^{\Gamma_{h}} \cong \mathcal{A}_{\text {bas }}^{2}\left(G_{2 h}, \operatorname{End}(V)\right)^{\Gamma_{h}}
$$

where the superscript $\Gamma_{h}$ denotes the $\Gamma_{h}$-invariant differential forms.

12.2. Proposition. Let $D_{2}$ be a rational boundary component of $D=G / K$. Then the connection form $\omega_{2}^{\mathrm{p}} \in \mathcal{A}^{1}\left(G_{2 h}, \operatorname{End}(V)\right)$ and the curvature form $\Omega_{2}^{\mathrm{p}} \in \mathcal{A}_{\text {bas }}^{2}\left(G_{2 h}, \operatorname{End}(V)\right)$ commute with the adjoint action of $\lambda_{2}\left(G_{2 \ell}\right) \subset G L(V)$.

12.3. Proof. The proof uses a double induction over boundary components $D_{2}$ in $D^{*}$. However, so as to avoid the horribly complicated notation which would arise in the proof, we rephrase the double induction as follows:

(1) By induction we assume the proposition has been proven for every rational boundary component $D_{2}^{\prime}$ of any Hermitian symmetric domain $D^{\prime}=G^{\prime} / K$ for which $\operatorname{dim}\left(D^{\prime}\right)<$ $\operatorname{dim}(D)$. (The case $\operatorname{dim}\left(D^{\prime}\right)=0$ is trivial.)

(2) For our given domain $D$, we assume the proposition has been proven for every rational boundary component $D_{1}$ of $D$ such that $\operatorname{dim}\left(D_{1}\right)<\operatorname{dim}\left(D_{2}\right)$. (The case $D_{1}=\phi$ is trivial.)

To prove Proposition 12.2 for $D_{2} \subset D^{*}$, it suffices to verify that $\lambda_{2}\left(G_{2 \ell}\right)$ commutes with the connection form of each of the connections appearing in the linear combination (12.1.1). The connection form $\omega_{2}^{\text {Nom }}$ of the Nomizu connection $\nabla_{2}^{\text {Nom }}$ is given by

$$
\omega_{2}^{\text {Nom }}\left(L_{g_{2 h} *}\left(\dot{g}_{2 h}\right)\right)=\lambda^{\prime}\left(\dot{k}_{2 h}\right)=\lambda_{2}^{\prime}\left(\dot{k}_{2 h}\right)
$$

for any $g_{2 h} \in G_{2 h}$ and for any $\dot{g}_{2 h} \in \mathfrak{g}_{2 h}$, where $\dot{g}_{2 h}=\dot{k}_{2 h}+\dot{p}_{2 h}$ is its Cartan decomposition. This commutes with $\lambda_{2}\left(G_{2 \ell}\right)$ since $G_{2 h}$ and $G_{2 \ell}$ commute.

Now consider any boundary component $D_{1} \prec D_{2}$ which appears in the sum (12.1.1). Let $P_{1}=\mathcal{U}_{1} G_{1 h} G_{1 \ell}$ be the rational parabolic subgroup which normalizes $D_{1}$. Decompose the intersection

$$
P=P_{1} \cap P_{2}=\mathcal{U}_{2}\left(\overline{\overline{\mathcal{U}}} G_{1 h} G_{\ell}^{\prime}\right) G_{2 \ell}
$$

according to (7.3.4), setting $P_{h}=\overline{\overline{\mathcal{U}}} G_{1 h} G_{\ell}^{\prime} \subset G_{2 h}$. Let $\nabla_{1}^{\mathrm{p}}$ be the patched connection on $E_{1} \rightarrow D_{1}$. According to Proposition 10.3, the connection form $\omega_{21}$ of the parabolically 
induced connection $\Phi_{21}^{*}\left(\nabla_{1}^{\mathrm{p}}\right)$ is given by

$$
\omega_{21}\left(L_{g *}\left(\dot{\bar{u}}+\dot{g}_{1 h}+\dot{g}_{\ell}^{\prime}\right)\right)=\lambda_{21}^{\prime}\left(\dot{g}_{\ell}^{\prime}\right)+A d\left(\lambda_{21}\left(g_{\ell}^{\prime}\right)^{-1}\right)\left(\omega_{1}^{\mathrm{p}}\left(L_{g_{1 h *}}\left(\dot{g}_{1 h}\right)\right)\right) .
$$

Here, $g=\overline{\bar{u}} g_{1 h} g_{\ell}^{\prime} \in P_{h}$ and $\dot{\bar{u}}+\dot{g}_{1 h}+\dot{g}_{\ell}^{\prime} \in \operatorname{Lie}\left(P_{h}\right)$ and $\lambda_{21}: K_{1 h} G_{\ell}^{\prime} \rightarrow \operatorname{GL}(V)$ is as in Proposition 8.2. Since $\operatorname{dim} Y<\operatorname{dim} X$ we may apply the first induction hypothesis and conclude that the adjoint action of $\lambda_{21}\left(G_{\ell}^{\prime}\right)$ commutes with the connection form $\omega_{1}^{\mathrm{p}} \in$ $\mathcal{A}^{1}\left(G_{1 h}, \operatorname{End}(V)\right)$. Hence, using Proposition 8.4,

$$
\omega_{21}\left(L_{g *}(\dot{\bar{u}})+\dot{g}_{1 h}+\dot{g}_{\ell}^{\prime}\right)=\lambda_{1}^{\prime}\left(\dot{g}_{\ell}^{\prime}\right)+\omega_{1}^{\mathrm{p}}\left(L_{g_{1 h *}}\left(\dot{g}_{1 h}\right)\right) .
$$

The group $G_{\ell}^{\prime}$ commutes with $G_{2 \ell}$ so the first term $\lambda_{1}^{\prime}\left(\dot{g}_{\ell}^{\prime}\right)$ commutes with $\lambda_{1}\left(G_{2 \ell}\right)$. By the second induction hypothesis, the connection form $\omega_{1}^{\mathrm{p}} \in \mathcal{A}^{1}\left(G_{1 h}\right.$, End $\left.(V)\right)$ also commutes with $\lambda_{1}\left(G_{1 \ell}\right)$. But $\lambda_{1}\left(G_{1 \ell}\right) \supset \lambda_{1}\left(G_{2 \ell}\right)=\lambda_{2}\left(G_{2 \ell}\right)$ by Proposition 8.4 again, which completes the proof that the connection form of the patched connection commutes with $\lambda_{2}\left(G_{2 \ell}\right)$.

12.4. Let $x \in X$ be a point near the boundary of $\bar{X}$. Then there is a maximal collection of strata $Y_{1}, Y_{2}, \ldots, Y_{t}$ such that $x \in T_{\epsilon_{0}}\left(Y_{i}\right)$ for each $i$, and we may assume they form a partial flag,

$$
Y_{1}<Y_{2}<\cdots<Y_{t}=X
$$

Let $W \leq X$ be the largest stratum in this collection such that $B_{W}^{\epsilon W}\left(\pi_{W}(x)\right) \neq 0$. Such a stratum exists since $B_{Y_{1}}^{\epsilon Y_{1}}\left(\pi_{Y_{1}}(x)\right)=1$.

Choosing $W$ in this way guarantees that, if $W \neq X$, then for every stratum $Z>W$ in this partial flag, at the point $\pi_{Z}(x)$ the connection $\nabla_{Z}^{\mathrm{p}}$ is an affine combination of connections induced from smaller strata and contains no contribution from $\nabla_{Z}^{\mathrm{Nom}}$, because $B_{Z}^{\epsilon Z}\left(\pi_{Z}(x)\right)=$ 0. To be precise:

12.5. Proposition. At the point $x \in X$,

$$
\sum_{W \leq \mathbf{S} \leq X} B_{\mathbf{S}}(x)=1
$$

and

$$
\nabla_{X}^{\mathrm{p}}(x)=\left(\sum_{W \leq \mathbf{S} \leq X} B_{\mathbf{S}}(x) \Phi_{\mathbf{S}}^{*}\right)\left(\nabla_{W}^{\mathrm{p}}\left(\pi_{W}(x)\right)\right)
$$

where the sum is over sub-chains $\mathbf{S}$ in the partial flag (12.4.1) which begin at $W$ and end at $X$.

12.6. Proof. By Lemma 11.5 applied to $\nabla_{W}^{\mathrm{p}}$, we need to show (at the point $x$ ) that

$$
\nabla_{X}^{\mathrm{p}}=\left(\sum_{W \leq \mathbf{S} \leq X} B_{\mathbf{S}} \Phi_{\mathbf{S}}^{*}\right)\left(\sum_{\mathbf{R} \leq W} B_{\mathbf{R}} \Phi_{\mathbf{R}}^{*}\left(B_{R(1)}^{\epsilon R(1)} \nabla_{R(1)}^{\mathrm{Nom}}\right)\right) .
$$


By Lemma $11.5, \nabla_{X}^{\mathrm{p}}(x)$ is a sum over chains $\mathbf{Z} \leq X$ of terms

$$
B_{\mathbf{Z}}(x) \Phi_{\mathbf{Z}}^{*}\left(B_{Z(1)}^{\epsilon Z(1)}\left(\pi_{Z(1)}(x)\right) \nabla_{Z(1)}^{\mathrm{Nom}}\right) .
$$

For any $\epsilon \leq \epsilon_{0}, B_{Z}^{\epsilon}(x)=0$ unless $Z$ occurs in the collection $\left\{Y_{1}, Y_{2}, \ldots, Y_{t}=X\right\}$. By assumption the term $B_{Z(1)}^{\epsilon Z(1)}\left(\pi_{Z(1)}(x)\right)$ also vanishes unless $Z(1) \leq W$. Therefore each chain $\mathbf{Z}=Z(1)<\cdots<X$ appearing in the sum may be assumed to occur as a sub-chain of $Y_{1}<Y_{2}<\cdots<Y_{t}=X$, and we may also assume the chain begins at $Z(1) \leq W$. We claim that if such a chain $\mathbf{Z}$ occurs with nonzero coefficient

$$
B_{\mathbf{Z}}(x) B_{Z(1)}^{\epsilon Z(1)}\left(\pi_{Z(1)}(x)\right) \neq 0
$$

then the stratum $W$ must appear in the chain. For if not, then $Z(k)<W<Z(k+1)$ for some $k$. But the term $B_{\mathbf{Z}}(x)$ contains a factor

$$
B_{Z(k)}^{\epsilon Z(k+1)}\left(\pi_{Z(k+1)}(x)\right) .
$$

Since $B_{W}^{\epsilon W}\left(\pi_{W}(x)\right) \neq 0$, this factor vanishes by Lemma 3.6, which proves the claim. Summarizing, every chain $\mathbf{Z}$ which occurs with nonzero coefficient in the sum may be described as

$$
R(1)<R(2)<\cdots<R(r)=W=S(1)<S(2)<\cdots<S(s)=X .
$$

The contribution to $\nabla_{X}^{\mathrm{p}}(x)$ in (11.5.2) from such a chain is the product of

$$
B_{S(s-1)}^{\epsilon X}\left(\pi_{X}(x)\right) \ldots B_{W}^{\epsilon S(2)}\left(\pi_{S(2)}(x)\right) \Phi_{X S(s-1)}^{*} \ldots \Phi_{S(2) W}^{*}
$$

with

applied to

$$
B_{R(r-1)}^{\epsilon W}\left(\pi_{W}(x)\right) \ldots B_{R(1)}^{\epsilon R(2)}\left(\pi_{R(2)}(x)\right) \Phi_{W R(r-1)}^{*} \ldots \Phi_{R(2) R(1)}^{*}
$$

$$
B_{R(1)}^{\epsilon R(1)}\left(\pi_{R(1)}(x)\right) \nabla_{R(1)}^{\text {Nom }} .
$$

However this product is exactly a single term in (12.6.1) and every such product occurs exactly once, which verifies (12.5.2).

A similar argument applies to the coefficients. By (11.5.1),

$$
\sum_{\mathbf{Z} \leq X} B_{\mathbf{Z}}(x) B_{Z(1)}^{\epsilon Z(1)}\left(\pi_{Z(1)}(x)\right)=1 .
$$

By the preceding argument, the only chains $\mathbf{Z}$ contributing to this sum are of the form (12.6.2), for which the term in (12.6.3) is the product of

$$
B_{S(s-1)}^{\epsilon X}\left(\pi_{X}(x)\right) \ldots B_{W}^{\epsilon S(2)}\left(\pi_{S(2)}(x)\right)
$$

with

$$
B_{R(r-1)}^{\epsilon W}\left(\pi_{W}(x)\right) \ldots B_{R(1)}^{\epsilon R(2)}\left(\pi_{R(2)}(x)\right) B_{R(1)}^{\epsilon R(1)}\left(\pi_{R(1)}(x)\right)
$$


which is a single term of

$$
\left(\sum_{W \leq \mathbf{S} \leq X} B_{\mathbf{S}}(x)\right) \cdot\left(\sum_{\mathbf{Z} \leq W} B_{\mathbf{Z}}\left(\pi_{W}(x)\right) B_{Z(1)}^{\epsilon Z(1)}\left(\pi_{Z(1)}(x)\right)\right)
$$

and every such product occurs exactly once. Therefore (12.6.4) is equal to 1. But the second factor in (12.6.4) is 1 by (11.5.1). This yields equation (12.5.1).

12.7. With these prelimintaries out of the way, let us begin the proof of Theorem 11.8. We must show that the Chern forms of the patched connection satisfy the control condition near each stratum of $\bar{X}$. Let $Y$ be such a stratum and let $x \in X \cap T_{Y}(\epsilon X / 2)$. We will verify the control condition relative to the stratum $Y$ at the point $x$. The point $x \in X$ lies in an intersection of $\epsilon_{0}$-tubular neighborhoods of a maximal collection of strata, which (we may assume) form a partial flag $Z_{1}<Z_{2}<\cdots<X$. Let $W$ be the largest stratum in this chain such that $B_{W}^{\epsilon W}\left(\pi_{W}(x)\right) \neq 0$. Then $W \leq Y$ by (3.2.2). Consider the subchain lying between $W$ and $Y$, which we shall denote by

$$
W=Y_{1}<Y_{2}<\cdots<Y_{t}=Y .
$$

Fix corresponding boundary components $D_{1} \prec D_{2} \prec \cdots \prec D_{t}$ and let $P_{1} \prec P_{2} \prec \ldots \prec P_{t}$ be their normalizing maximal parabolic subgroups. Set $P=P_{1} \cap P_{2} \cap \ldots \cap P_{t}$. Let $\nabla_{W}^{\mathrm{p}}$ be the patched connection on $E_{W}^{\prime} \rightarrow W$. According to Proposition 12.5,

$$
\nabla_{X}^{\mathrm{p}}(x)=\left(\sum_{W \leq \mathbf{S} \leq X} B_{\mathbf{S}}(x) \Phi_{\mathbf{S}}^{*}\right) \nabla_{W}^{\mathrm{p}}\left(\pi_{W}(x)\right) .
$$

However the only chains $\mathbf{S}=\{S(1)<\cdots<S(s)\}$ which occur with nonzero coefficient in this sum satisfy

$$
\{S(1), S(2), \ldots, S(s-1)\} \subset\left\{Y_{1}, Y_{2}, \ldots, Y_{t}\right\}, S(1)=W=Y_{1}, S(s)=X
$$

for the following reason. Suppose a chain $\mathbf{S}$ contains a stratum larger than $Y$ (but not equal to $X)$. Let $Z$ be the largest such stratum occurring in $\mathbf{S}$. Then the first factor in $B_{\mathbf{S}}(x)$ is $B_{Z}^{\epsilon X}(x)$, cf. (11.4.1). By assumption, $x \in T_{Y}(\epsilon X / 2)$. So by (3.2.2) (with $\epsilon=\epsilon X$ and where the roles of $Y$ and $Z$ are reversed), $B_{Z}^{\epsilon X}(x)=0$.

If $W=Y$ then (12.7.1) becomes $\nabla_{X}^{\mathrm{p}}=\Phi_{X Y}^{*}\left(\nabla_{Y}^{\mathrm{p}}\right)$ so by Corollary 10.5 and Proposition 12.2 the curvature form $\Omega_{X}^{\mathrm{p}}$ of $\nabla_{X}^{\mathrm{p}}$ is controlled with respect to $Y$. So the same is true of every Chern form which proves Theorem 11.8 in this case.

Therefore we may assume that $W<Y$. As in $\S 12.1$ the connection $\nabla_{W}^{\mathrm{p}}$ on $E_{W}^{\prime} \rightarrow W$ pulls back to a $\Gamma_{h}$-invariant connection $\nabla_{1}^{\mathrm{p}}$ on $E_{1} \rightarrow D_{1}$ and the connection $\nabla_{X}^{\mathrm{p}}$ on $E^{\prime} \rightarrow X$ pulls back to a $\Gamma$-invariant connection $\nabla^{\mathrm{p}}$ on $E \rightarrow D$. As in (12.1.2) we identify the curvature form $\Omega_{X}^{\mathrm{p}}$ of $\nabla_{X}^{\mathrm{p}}$ with the curvature form $\Omega^{\mathrm{p}}$ of $\nabla^{\mathrm{p}}$ under the canonical isomorphism

$$
\mathcal{A}^{2}\left(X, \operatorname{End}\left(E^{\prime}\right)\right) \cong \mathcal{A}^{2}(D, \operatorname{End}(E))^{\Gamma} \cong \mathcal{A}_{\text {bas }}^{2}(G, \operatorname{End}(V))^{\Gamma}
$$


Similarly identify the curvature $\Omega_{1}^{\mathrm{p}}$ of $\nabla_{1}^{\mathrm{p}}$ with the curvature $\Omega_{W}^{\mathrm{p}}$ of $\nabla_{W}^{\mathrm{p}}$. Choose a lift $\tilde{x} \in D$ of $x$, which lies in the intersection

$$
U_{D_{1}}\left(\epsilon_{0}\right) \cap U_{D_{2}}\left(\epsilon_{0}\right) \cap \ldots \cap U_{D_{t}}\left(\epsilon_{0}\right)
$$

of $\Gamma$-parabolic neighborhoods of the boundary components $D_{1} \prec D_{2} \prec \ldots \prec D_{t}$. Let $\pi: D \rightarrow D_{1}$ denote the canonical projection.

12.8. Lemma. For any tangent vectors $U, V \in T_{\tilde{x}} D$,

$$
\Omega^{\mathrm{p}}(U, V)=\pi^{*} \Omega_{1}^{\mathrm{p}}(U, V)+n \in \operatorname{End}(V)
$$

for some nilpotent element $n \in \lambda_{1}^{\prime}\left(\mathfrak{g}_{1 \ell}\right)$. (Here, $P_{1}=\mathcal{U}_{P_{1}} G_{1 h} G_{1 \ell} ; \mathfrak{g}_{1 \ell}=\operatorname{Lie}\left(G_{1 \ell}\right) ;$ and $\lambda_{1}$ is the extension (Proposition 8.2) of the representation $\lambda \mid K \cap P_{1}$.)

12.9. Proof. We will compute the curvature $\Omega_{X}^{\mathrm{p}}$ of $\nabla_{X}^{\mathrm{p}}$. Each chain $\mathbf{S}$ satisfying (12.7.2) corresponds also to a chain of rational boundary components $D_{\mathbf{S}(1)} \prec D_{\mathbf{S}(2)} \prec \ldots \prec D_{\mathbf{S}(\mathbf{s})}$ which begin at $D_{1}=D_{\mathbf{S}(\mathbf{1})}$ (the boundary component corresponding to the stratum $W$ ) and end at $D=D_{\mathbf{S}(\mathbf{s})}$. Let $\widetilde{B}_{\mathbf{S}}=q^{*} B_{\mathbf{S}}$ denote the pullback to $D$. It follows from Proposition 12.5 that $\sum_{\mathbf{S}} \widetilde{B}_{\mathbf{S}}(\tilde{x})=1$ (where the sum is over all chains $\mathbf{S}$ which appear in (12.7.1)). Choose any ordered labeling of the chains $\mathbf{S}$ which appear in $(12.7 .1)$, say $\mathbf{S}_{\mathbf{1}}, \mathbf{S}_{\mathbf{2}}, \ldots, \mathbf{S}_{\mathbf{M}}$.

Let $U, V \in T_{\tilde{x}} D$. Pulling back the equation (12.7.1) to $D$ and using Lemma 6.2 gives:

$$
\begin{aligned}
\Omega^{\mathrm{p}}(U, V)= & \sum_{i=1}^{M} \widetilde{B}_{\mathbf{S}_{\mathbf{i}}} \Phi_{\mathbf{S}_{\mathbf{i}}}^{*}\left(\Omega_{1}^{\mathrm{p}}\right)(U, V)+\sum_{i=1}^{M-1} d \widetilde{B}_{\mathbf{S}_{i}} \wedge\left(\Phi_{\mathbf{S}_{i}}^{*} \omega_{1}^{\mathrm{p}}-\Phi_{\mathbf{S}_{M}}^{*} \omega_{1}^{\mathrm{p}}\right)(U, V) \\
& -\sum_{i<j} \widetilde{B}_{\mathbf{S}_{i}}(x) \widetilde{B}_{\mathbf{S}_{j}}(x)\left[\Phi_{\mathbf{S}_{i}}^{*} \omega_{1}^{\mathrm{p}}(U)-\Phi_{\mathbf{S}_{j}}^{*} \omega_{1}^{\mathrm{p}}(U), \Phi_{\mathbf{S}_{i}}^{*} \omega_{1}^{\mathrm{p}}(V)-\Phi_{\mathbf{S}_{j}}^{*} \omega_{1}^{\mathrm{p}}(V)\right]
\end{aligned}
$$

where $\Phi_{\mathbf{S}}^{*}\left(\omega_{1}^{\mathrm{p}}\right)$ denotes the connection form of $\Phi_{\mathbf{S}}^{*}\left(\nabla_{1}^{\mathrm{p}}\right)$ and where $\Phi_{\mathbf{S}}^{*}\left(\Omega_{1}^{\mathrm{p}}\right)$ denotes its curvature. Because of Proposition 12.2 we may apply Proposition 10.9 , especially equation (10.9.2) to obtain $\Phi_{\mathbf{S}_{\mathbf{i}}}^{*}\left(\Omega_{1}^{\mathrm{p}}\right)=\pi^{*}\left(\Omega_{1}^{\mathrm{p}}\right)$. Therefore the first term in (12.9.1) is

$$
\sum_{i=1}^{M} \widetilde{B}_{\mathbf{S}_{\mathbf{i}}} \pi^{*}\left(\Omega_{1}^{\mathrm{p}}\right)(U, V)=\pi^{*}\left(\Omega_{1}^{\mathrm{p}}\right)(U, V)
$$

by (12.5.1). In order to evaluate the remaining terms we must first compute the connection forms $\Phi_{\mathbf{S}_{\mathbf{i}}}^{*}\left(\omega_{1}^{\mathrm{p}}\right)$. Suppose that $\mathbf{S}$ is a chain satisfying (12.7.2). For $1 \leq j \leq s-1$ let $P_{S(j)}$ be the corresponding normalizing maximal parabolic subgroup and set $Q=P_{S(1)} \cap P_{S(2)} \cap \ldots \cap$ $P_{S(s-1)}$. Then $P \subset Q$ and $P^{b}=Q^{b}=P_{1}$ which implies (as in $\S 7.3$ ) that $P \subset Q \subset P_{1}$ have compatible decompositions,

$$
\begin{aligned}
P_{1} & =\mathcal{U}_{1} G_{1 h} G_{1 \ell} \\
Q & =\mathcal{U}_{1} G_{1 h}\left(\mathcal{U}_{P_{1} Q} G_{Q \ell}\right) \text { with } \mathcal{U}_{Q}=\mathcal{U}_{1} \mathcal{U}_{P_{1} Q} \\
P & =\mathcal{U}_{1} G_{1 h} \mathcal{U}_{P_{1} Q}\left(\mathcal{U}_{Q P} G_{P \ell}\right) \text { with } \mathcal{U}_{P}=\mathcal{U}_{1} \mathcal{U}_{P_{1} Q} \mathcal{U}_{Q P}
\end{aligned}
$$


Here, $\mathcal{U}_{Q P} G_{P \ell}$ is the parabolic subgroup of $G_{Q \ell}$ determined by $P \subset Q$. We also note that

$$
\mathcal{U}_{P_{1} P}=\mathcal{U}_{P_{1} Q} \mathcal{U}_{Q P}
$$

is the unipotent radical of the parabolic subgroup $\nu_{1 \ell}(P) \subset G_{1 \ell}$ determined by $P$ (where $\nu_{1 \ell}: P_{1} \rightarrow G_{1 \ell}$ is the projection). Let $\mathfrak{N}_{P_{1} P}$ denote its Lie algebra.

By Proposition 12.2, the connection form $\omega_{1}^{\mathrm{p}} \in \mathcal{A}^{1}\left(G_{1 h}\right.$, End $\left.(V)\right)$ commutes with the adjoint action of $\lambda_{1}\left(G_{1 \ell}\right) \subset G L(V)$. So we may apply Proposition 10.9 to determine $\Phi_{\mathbf{S}}^{*}\left(\omega_{1}^{\mathrm{p}}\right)$. Let $g=u_{P} g_{1 h} g_{P \ell} \in P=\mathcal{U}_{P} G_{1 h} G_{P \ell}$ and let

$$
\dot{g}=\dot{u}_{1}+\dot{g}_{1 h}+\dot{u}_{P_{1} Q}+\dot{u}_{Q P}+\dot{g}_{P \ell} \in \operatorname{Lie}\left(\mathcal{U}_{1} G_{1 h} \mathcal{U}_{P_{1} Q} \mathcal{U}_{Q P} G_{P \ell}\right) .
$$

Apply Proposition 10.9 using $\dot{u}_{Q}=\dot{u}_{1}+\dot{u}_{P_{1} Q} \in \operatorname{Lie}\left(\mathcal{U}_{Q}\right)$ and $\dot{g}_{Q \ell}=\dot{u}_{Q P}+\dot{g}_{P \ell} \in \mathfrak{g}_{Q \ell}$ to find:

$$
\left.\Phi_{\mathbf{S}}^{*}\left(\omega_{1}^{\mathrm{p}}\right)\left(L_{g *}(\dot{g})\right)=\omega_{1}^{\mathrm{p}}\left(L_{g_{1 h} *} \dot{g}_{1 h}\right)\right)+\lambda_{1}^{\prime}\left(\dot{u}_{Q P}\right)+\lambda_{1}^{\prime}\left(\dot{g}_{P \ell}\right) .
$$

Moreover, $\lambda_{1}^{\prime}\left(\dot{u}_{Q P}\right) \in \lambda_{1}^{\prime}\left(\mathfrak{N}_{P_{1} P}\right) \subset \lambda_{1}^{\prime}\left(\mathfrak{g}_{1 \ell}\right)$.

Now suppose that $\mathbf{R}$ is another chain in the sum (12.7.1) which makes a nonzero contribution $\Phi_{\mathbf{R}}^{*} \nabla_{1}^{\mathrm{p}}=d+\Phi_{\mathbf{R}}^{*}\left(\omega_{1}^{\mathrm{p}}\right)$ to the connection $\nabla^{\mathrm{p}}$, say, $W=R(1)<R(2)<\cdots<R(r-1)<X$. Then $Q^{\prime}=P_{R(1)} \cap P_{R(2)} \cap \ldots \cap P_{R(r-1)}$ and $P$ also have compatible decompositions:

$$
\begin{aligned}
Q^{\prime} & =\mathcal{U}_{1} G_{1 h}\left(\mathcal{U}_{P_{1} Q^{\prime}} G_{Q^{\prime} \ell}\right) \text { with } \mathcal{U}_{Q^{\prime}}=\mathcal{U}_{1} \mathcal{U}_{P_{1} Q^{\prime}} \\
P & =\mathcal{U}_{1} G_{1 h} \mathcal{U}_{P_{1} Q^{\prime}}\left(\mathcal{U}_{Q^{\prime} P} G_{P \ell}\right) \text { with } \mathcal{U}_{P}=\mathcal{U}_{1} \mathcal{U}_{P_{1} Q^{\prime}} \mathcal{U}_{Q^{\prime} P}
\end{aligned}
$$

The same element $\dot{g} \in \operatorname{Lie}(P)$ decomposes as

$$
\dot{g}=\dot{u}_{1}+\dot{g}_{1 h}+\dot{u}_{P_{1} Q^{\prime}}+\dot{u}_{Q^{\prime} P}+\dot{g}_{P \ell} .
$$

So the same argument gives $\Phi_{\mathbf{R}}^{*}\left(\omega_{1}^{\mathrm{p}}\right)\left(L_{g *}(\dot{g})\right)=\omega_{1}^{\mathrm{p}}\left(L_{g_{1 h} *}\left(\dot{g}_{1 h}\right)\right)+\lambda_{1}^{\prime}\left(\dot{u}_{Q^{\prime} P}\right)+\lambda_{1}^{\prime}\left(\dot{g}_{P \ell}\right)$. We conclude that:

$$
\left(\Phi_{\mathbf{S}}^{*} \omega_{1}^{\mathrm{p}}-\Phi_{\mathbf{R}}^{*} \omega_{1}^{\mathrm{p}}\right)\left(L_{g *}(\dot{g})\right)=\lambda_{1}^{\prime}\left(\dot{u}_{Q P}-\dot{u}_{Q^{\prime} P}\right) \in \lambda_{1}^{\prime}\left(\mathfrak{N}_{P_{1} P}\right) \subset \lambda_{1}^{\prime}\left(\mathfrak{g}_{1 \ell}\right) .
$$

Consequently each term in the sum (12.9.1) (except for the first) lies in $\lambda_{1}^{\prime}\left(\mathfrak{N}_{P_{1} P}\right)$.

12.10. Completion of the proof. Using Lemma 12.8, at the point $\tilde{x}$ we may write

$$
\Omega^{\mathrm{p}}(U, V)=\pi^{*}\left(\Omega_{1}^{\mathrm{p}}\right)(U, V)+n
$$

where $n \in \lambda_{1}^{\prime}\left(G_{1 \ell}\right)$ is nilpotent and in fact lies in $\lambda_{1}^{\prime}\left(\mathfrak{N}_{P_{1} P}\right)$. Moreover, by Proposition 12.2, the curvature $\Omega_{1}^{\mathrm{p}}$ commutes with $n$. If $f: \operatorname{End}(V) \rightarrow \mathbb{C}$ is any $A d$-invariant polynomial, it follows from Lemma 6.4 that $f\left(\Omega^{\mathrm{p}}(U, V)\right)=f\left(\Omega_{1}^{\mathrm{p}}\left(\pi_{*} U, \pi_{*} V\right)\right)$ hence also $f\left(\Omega_{X}^{\mathrm{p}}(U, V)\right)=$ $f\left(\Omega_{W}^{\mathrm{p}}\left(\pi_{X W *} U, \pi_{X W *} V\right)\right)$. So it follows from (6.3.1) that the corresponding characteristic form is controlled relative to $W$. This completes the proof of Theorem 11.8. 


\section{TOROIDAL COMPACTIFICATION}

13.1. Throughout this section we assume that $G=\mathbf{G}(\mathbb{R})$ is the set of real points of a connected semisimple algebraic group $\mathbf{G}$ defined over $\mathbb{Q}$, that $D=G / K$ is a Hermitian symmetric space, $\Gamma \subset \mathbf{G}(\mathbb{Q})$ is a neat arithmetic group, $X=\Gamma \backslash G / K$ is the corresponding locally symmetric space with Baily-Borel Satake compactification $\bar{X}=\Gamma \backslash D^{*}$. Fix a representation $\lambda: K \rightarrow G L(V)$ on some complex vector space $V$ and let $E=G \times_{K} V$ be the corresponding homogeneous vector bundle on $D$, and $E^{\prime}=\Gamma \backslash E$ the automorphic vector bundle on $X$. Choose a system of control data on the Baily-Borel compactification $\bar{X}$ and a partition of unity as in $\S 3$, and let $\nabla_{X}^{\mathrm{p}}$ denote the resulting patched connection on $E^{\prime} \rightarrow X$. For each $i$, the Chern form $\sigma^{i}\left(\nabla_{X}^{\mathrm{p}}\right) \in \Omega_{\pi}^{\bullet}(\bar{X} ; \mathbb{C})(\operatorname{cf} \S 6.3)$ is a controlled differential form on $\bar{X}$ so it determines a cohomology class $\bar{c}^{i}\left(E^{\prime}\right)=\left[\sigma^{i}\left(\nabla_{X}^{\mathrm{p}}\right)\right] \in H^{2 i}(\bar{X} ; \mathbb{C})$.

We also fix a nonsingular toroidal compactification $\bar{X}_{\Sigma}$. This corresponds to a $\Gamma$-compatible collection of simplicial polyhedral cone decompositions $\Sigma_{F}$ of certain self adjoint homogeneous cones. These compactifications were constructed in [AMRT] and are reviewed in [Har3], [HZ1], [FC], [Na]. In [Mu1], D. Mumford shows that the automorphic vector bundle $E^{\prime} \rightarrow X$ admits a canonical extension $\bar{E}_{\Sigma}^{\prime}$ over the toroidal compactification $\bar{X}_{\Sigma}$. In [Har3] Theorem 4.2, M. Harris shows that Mumford's canonical extension coincides with Deligne's canonical extension [D] (for an appropriately chosen flat connection with unipotent monodromy).

The identity mapping $X \rightarrow X$ has a unique continuous extension, $\tau: \bar{X}_{\Sigma} \rightarrow \bar{X}$ of $X$, and this is a resolution of singularities.

13.2. Theorem. The patched connection $\nabla_{X}^{\mathrm{p}}$ on $E^{\prime} \rightarrow X$ extends to a smooth connection $\bar{\nabla}_{\Sigma}^{\mathrm{p}}$ on $\bar{E}_{\Sigma}^{\prime} \rightarrow \bar{X}_{\Sigma}$. Moreover for each $i$,

$$
\tau^{*} \bar{c}^{i}\left(E^{\prime}\right)=\tau^{*}\left(\left[\sigma^{i}\left(\nabla_{X}^{\mathrm{p}}\right)\right]\right)=\left[\sigma^{i}\left(\bar{\nabla}_{\Sigma}^{\mathrm{p}}\right)\right]=c^{i}\left(\bar{E}_{\Sigma}^{\prime}\right) \in H^{2 i}\left(\bar{X}_{\Sigma} ; \mathbb{C}\right) .
$$

The proof will appear in Section 14. S. Zucker has pointed out that it follows from mixed Hodge theory that the image of $\bar{c}^{i}\left(E^{\prime}\right)$ in $\operatorname{Gr}_{2 i}^{W} H^{2 i}(\bar{X} ; \mathbb{C})$ is uniquely determined by (13.2.1).

13.3. Proportionality theorem. Fix representations $\lambda_{j}: K \rightarrow G L\left(V_{j}\right)$ for $j=1,2, \ldots, r$ and fix nonnegative integers $I=\left(i_{1}, i_{2}, \ldots i_{r}\right)$ with $i_{1}+i_{2}+\ldots+i_{r}=n=\operatorname{dim}(D)$. For $j=1,2, \ldots, r$ let $E_{j}^{\prime}=\Gamma \backslash G \times_{K} V_{j} \rightarrow X$ be the resulting automorphic vector bundle on $X$ and let $\check{E}_{j}=G_{u} \times_{K} V_{j}$ be the corresponding vector bundle on the compact dual symmetric space $\check{D}=G_{u} / K$ (where $G_{u}$ is a compact real form of $\mathbf{G}$ containing $K$ ). Define "generalized" Chern numbers

$$
\check{c}^{I}\left(\lambda_{1}, \lambda_{2}, \ldots, \lambda_{r}\right)=\left(c^{i_{1}}\left(\check{E}_{1}\right) \cup c^{i_{2}}\left(\check{E}_{2}\right) \cup \ldots \cup c^{i_{r}}\left(\check{E}_{r}\right)\right) \cap[\check{D}] \in \mathbb{Z}
$$

and

$$
\left(\bar{c}^{I}\left(\lambda_{1}, \lambda_{2}, \ldots, \lambda_{r}\right)=\bar{c}^{i_{1}}\left(\nabla_{1}^{\mathrm{p}}\right) \cup \bar{c}^{i_{2}}\left(\nabla_{2}^{\mathrm{p}}\right) \cup \ldots \cup c^{i_{r}}\left(\nabla_{r}^{\mathrm{p}}\right)\right) \cap[\bar{X}] \in \mathbb{C}
$$


where $\nabla_{j}^{\mathrm{p}}$ denotes the patched connection on $E_{j}^{\prime} \rightarrow X$ and where $[\bar{X}] \in H_{2 n}(\bar{X} ; \mathbb{C})$ denotes the fundamental class of the Baily-Borel compactification. Let $v(\Gamma) \in \mathbb{Q}$ denote the constant which appears in the proportionality theorem of Hirzebruch [Hr1], [Mu1].

13.4. Proposition. For any choice $\lambda_{1}, \lambda_{2}, \ldots, \lambda_{r}$ of representations and for any partition $I=\left(i_{1}, i_{2}, \ldots, i_{r}\right)$ of $n=\operatorname{dim}_{\mathbb{C}}(D)$ we have

$$
\bar{c}^{I}\left(\lambda_{1}, \lambda_{2}, \ldots, \lambda_{r}\right)=v(\Gamma) \check{c}^{I}\left(\lambda_{1}, \lambda_{2}, \ldots, \lambda_{r}\right) .
$$

13.5. Proof. Each of the vector bundles $E_{j}^{\prime}$ has a canonical extension $\bar{E}_{j, \Sigma}^{\prime} \rightarrow \bar{X}_{\Sigma}$. The same proof as in [Mu1] (which is the same proof as in [Hr1]) (cf. [Hr3]) shows that the Chern classes of these extended bundles satisfy the proportionality formula

$$
\left(c^{i_{1}}\left(\bar{E}_{1, \Sigma}^{\prime}\right) \cup c^{i_{2}}\left(\bar{E}_{2, \Sigma}^{\prime}\right) \cup \ldots \cup c^{i_{r}}\left(\bar{E}_{r, \Sigma}^{\prime}\right)\right) \cap\left[\bar{X}_{\Sigma}\right]=v(\Gamma) \check{c}^{I}\left(\lambda_{1}, \lambda_{2}, \ldots, \lambda_{r}\right) .
$$

The result now follows immediately from Theorem 13.2 .

\section{Proof of Theorem 13.2}

14.1. Fix $\epsilon \leq \epsilon_{0}$. We claim that the partition of unity (3.3.1) $\sum_{Z \leq X} B_{Z}^{\epsilon}=1$ on $\bar{X}$ pulls back to a smooth partition of unity on $\bar{X}_{\Sigma}$. Fix a pair of strata $Y, Z$ of $\bar{X}$. We must verify that $\tau^{*} B_{Z}^{\epsilon}$ is smooth near $\tau^{-1}(Y)$. It can be shown that the mapping $\tau: \bar{X}_{\Sigma} \rightarrow \bar{X}$ is a complex analytic morphism between complex analytic varieties, as is the projection $\pi_{Y}: T_{Y}(\epsilon) \rightarrow Y$. It follows that the composition $\pi_{Y} \tau: \tau^{-1} T_{Y}(\epsilon) \rightarrow Y$ is a complex analytic morphism between smooth complex varieties, so it is smooth. If the stratum $Z$ is not comparable to $Y$ or if $Z>Y$ then by $(3.2 .2), B_{Z}^{\epsilon}$ vanishes on $T_{Y}(\epsilon / 2)$. Therefore $\tau^{*} B_{Z}^{\epsilon}$ vanishes on $\tau^{-1}\left(T_{Y}(\epsilon / 2)\right)$ so it is smooth. If $Z \leq Y$ then by (3.2.4) (when $Z<Y$ ) and (3.2.5) (when $Z=Y$ )

$$
\tau^{*} B_{Z}^{\epsilon}(x)=B_{Z}^{\epsilon} \pi_{Y} \tau(x) \text { for all } x \in \tau^{-1}\left(T_{Y}(\epsilon / 2)\right),
$$

so $\tau^{*} B_{Z}^{\epsilon}$ is smooth in this open set.

14.2. We may assume that $\mathbf{G}$ is simple over $\mathbb{Q}$. The "boundary" $\bar{X}_{\Sigma}-X$ of the toroidal compactification has a distinguished covering by open sets $U_{Y}$, one for each stratum $Y \subset \bar{X}$ of the Baily-Borel compactification, such that $\tau\left(U_{Y}\right) \subset \bar{X}$ is a neighborhood of $Y$, and for which the restriction $\bar{E}_{\Sigma}^{\prime} \mid U_{Y}$ arises from an automorphy factor ([HZ1] $\left.\S 3.3\right)$.

14.3. Proposition. For any smooth connection $\nabla_{Y}$ on $\left(E_{Y}^{\prime}, Y\right)$ the parabolically induced connection $\Phi_{X Y}^{*}\left(\nabla_{Y}\right)$ (which is defined only on $E^{\prime}\left|\left(U_{Y} \cap X\right)=\bar{E}_{\Sigma}^{\prime}\right|\left(U_{Y} \cap X\right)$ ) extends canonically to a smooth connection (which we denote by $\bar{\Phi}_{X Y}^{*}\left(\nabla_{Y}\right)$ ) on $\bar{E}_{\Sigma}^{\prime} \mid U_{Y}$. 
14.4. Proof of Theorem 13.2. We may assume that $\epsilon_{0}>0$ was chosen so small that $T_{Y}\left(\epsilon_{0}\right) \subset \tau\left(U_{Y}\right)$ for each stratum $Y<X$ of $\bar{X}$. By $\S 14.1$ the partition of unity which is used to construct the patched connection $\nabla_{X}^{\mathrm{p}}$ extends to a smooth partition of unity on $\bar{X}_{\Sigma}$. Hence, using Proposition 14.3,

$$
\bar{\nabla}_{\Sigma}^{\mathrm{p}}=\tau^{*} B_{X}^{\epsilon X}+\sum_{Y<X} \tau^{*} B_{Y}^{\epsilon X} \bar{\Phi}_{X Y}^{*}\left(\nabla_{Y}^{\mathrm{p}}\right)
$$

is a smooth connection on $\bar{E}_{\Sigma}^{\prime} \rightarrow \bar{X}_{\Sigma}$ which coincides with the patched connection $\nabla_{X}^{\mathrm{p}}$ on $E^{\prime} \rightarrow X$. Therefore its Chern forms are smooth and everywhere defined and they restrict to the Chern forms of $\nabla_{X}^{\mathrm{p}}$. It follows from Lemma 4.5 that each Chern class of $\bar{\nabla}_{\Sigma}^{\mathrm{p}}$ is the pullback of the corresponding Chern class of $\nabla_{X}^{\mathrm{p}}$.

The remainder of $\S 13$ is devoted to the proof of Proposition 14.3 which is essentially proven in [HZ1] (3.3.9) (following [Har2]). We will now verify the details.

14.5. Let us recall the construction $[\mathrm{AMRT}]$ of the toroidal compactification. Fix a rational boundary component $F$ with normalizing parabolic subgroup $P=\mathcal{U} G_{h} G_{\ell}$ and let $Y=$ $\Gamma_{h} \backslash F \subset \bar{X}$ denote the corresponding stratum in the Baily-Borel compactification of $X=$ $\Gamma \backslash D$. Let $Z_{F}=\operatorname{Center}(\mathcal{U})$ and $\mathfrak{z}=\operatorname{Lie}\left(Z_{F}\right)$. The vector space $\mathfrak{z}$ is preserved under the adjoint action of $G_{\ell}$ and contains a unique open orbit $C_{F} \subset \mathfrak{z}$; it is a rationally defined self adjoint homogeneous cone. Its Satake compactification $C_{F}^{*}$ consists of $C_{F}$ together with all its rational boundary components (in the Satake topology). The toroidal compactification is associated to a collection $\Sigma=\left\{\Sigma_{F}\right\}$ of rational polyhedral cone decompositions of the various $C_{F}^{*}$ which are compatible under $\Gamma$.

Let $\check{D}$ denote the compact dual symmetric space (so $\check{D}=\mathbf{G}(\mathbb{C}) / K(\mathbb{C}) P^{-}$in the notation of Proposition 8.2; cf.[AMRT], [Sat]) and let $\beta: D \rightarrow \check{D}$ denote the Borel embedding. Set $D_{F}=Z_{F}(\mathbb{C}) \cdot \beta(D)$. The domain $D_{F}$ is homogeneous under $P . Z_{F}(\mathbb{C})$ and it admits "Siegel coordinates" $D_{F} \cong Z_{F}(\mathbb{C}) \times \mathbb{C}^{a} \times F$ in which the subset $\beta(D) \subset D_{F}$ is defined by a certain well known inequality ([AMRT] p. 239, [Sat] $\S I I I(7.4))$. Now consider the commutative diagram [HZ1] (1.2.5), reproduced in figure 5.

Here, $\Gamma_{F}^{\prime}=\Gamma \cap\left(G_{h} \mathcal{U}\right)$ and $M_{F}^{\prime}=\Gamma_{F}^{\prime} \backslash D_{F}$. The algebraic torus $T_{F}=Z_{F}(\mathbb{C}) /\left(\Gamma \cap Z_{F}\right)$ acts on $M_{F}^{\prime}$ with quotient $A_{F}$, which is in turn an abelian scheme over $Y$. The choice $\Sigma_{F}$ of polyhedral cone decomposition determines a torus embedding $T_{F} \hookrightarrow T_{F, \Sigma}$ and a partial compactification $M_{F, \Sigma}^{\prime}=M_{F}^{\prime} \times_{T_{F}} T_{F, \Sigma}$ of $M_{F}^{\prime}$. Let $D_{F, \Sigma}$ denote the interior of the closure of $M_{F}^{\prime}=\Gamma_{F}^{\prime} \backslash D$ in $M_{F, \Sigma}^{\prime}$. The quotient mapping $\Gamma_{F}^{\prime} \backslash D \rightarrow X$ extends to a local isomorphism $\varphi_{F, \Sigma}: D_{F, \Sigma} \rightarrow \bar{X}_{\Sigma}$ (cf. [AMRT] p. 250). In other words, $\varphi_{F, \Sigma}$ is an open analytic mapping with discrete fibers which, near the boundary, induces an embedding $D_{F, \Sigma} /\left(\Gamma_{F}^{\prime} \backslash \Gamma_{P}\right) \hookrightarrow \bar{X}_{\Sigma}$ whose image is the neighborhood $U_{Y}$ referred to in $\S 14.2$. The mappings $\varphi_{F, \Sigma}$ for the various strata $Y \subset \bar{X}$ cover the boundary of $\bar{X}_{\Sigma}$. The composition $\theta_{1} \theta_{2} \mid D: D \rightarrow F$ coincides with the canonical projection $\pi$. 


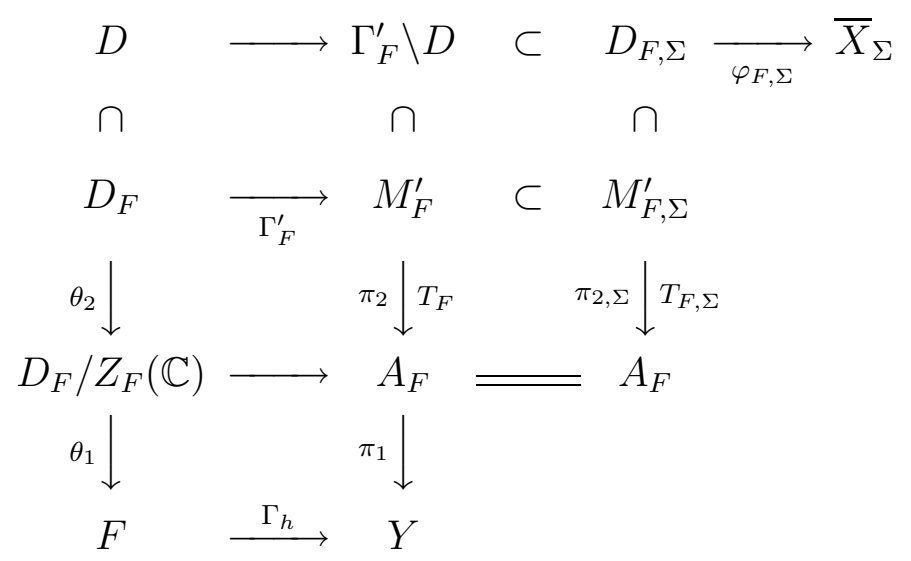

FIGURE 5. Toroidal compactification

14.6. Each of the spaces in Figure 5 comes equipped with a vector bundle and most of the mappings in this diagram are covered by vector bundle isomorphisms. We will make the following notational convention: If $E_{1} \rightarrow M_{1}$ and $E_{2} \rightarrow M_{2}$ are (smooth) vector bundles, $\alpha: M_{1} \rightarrow M_{2}$ is a (smooth) mapping, and $\Phi: E_{1} \rightarrow E_{2}$ is a vector bundle mapping which induces an isomorphism $E_{1} \cong \alpha^{*}\left(E_{2}\right)$, then we will write $\Phi:\left(E_{1}, M_{1}\right) \sim\left(E_{2}, M_{2}\right)$ and refer to $\Phi$ as being a vector bundle isomorphism which covers $\alpha$.

As in [Mu1], complexify $\lambda: K \rightarrow G L(V)$ and extend it trivially over $P^{-}$to obtain a representation $\tilde{\lambda}: K(\mathbb{C}) P^{-} \rightarrow G L(V)$. The homogeneous vector bundle $E=G \times{ }_{K} V$ has a canonical extension

$$
\check{E}=\mathbf{G}(\mathbb{C}) \times_{K(\mathbb{C}) P^{-}} V
$$

over the compact dual symmetric space $\check{D}$. Its restriction $E_{F}$ to $D_{F}$ is a $P Z_{F}(\mathbb{C})$-homogeneous bundle, and it passes to a vector bundle $E_{F}^{\prime} \rightarrow M_{F}^{\prime}$ upon dividing by $\Gamma_{F}^{\prime}$. The restriction $E_{F}^{\prime} \mid\left(\Gamma_{F}^{\prime} \backslash D\right)$ coincides with the vector bundle obtained from the homogeneous vector bundle $E=G \times_{K} V$ upon dividing by $\Gamma_{F}^{\prime}$. We will denote this restriction also by $\left(E_{F}^{\prime}, \Gamma_{F}^{\prime} \backslash D\right)$.

Define $\widetilde{E}=P . Z_{F}(\mathbb{C}) \times_{K_{P} . Z_{F}(\mathbb{C})} V$. This vector bundle on $D_{F} / Z_{F}(\mathbb{C})$ is homogeneous under $P . Z_{F}(\mathbb{C})$ and it passes to a vector bundle $E_{F}^{A} \rightarrow A_{F}$ upon dividing by $\Gamma_{F}^{\prime}$. As in [HZ1] (3.2.1) and (3.3.5), there is a canonical vector bundle isomorphism

$$
\psi:\left(E_{F}^{\prime}, M_{F}^{\prime}\right) \sim\left(E_{F}^{A}, A_{F}\right)
$$

which covers $\pi_{2}$. In fact, the isomorphism $\psi$ is obtained from the canonical isomorphism of $P . Z_{F}(\mathbb{C})$-homogeneous vector bundles,

$$
\Psi:\left(E_{F}, D_{F}\right) \sim\left(\widetilde{E}, D_{F} / Z_{F}(\mathbb{C})\right)
$$

which covers $\theta_{2}$ and which is given by the quotient mapping

$$
E_{F}=P . Z_{F}(\mathbb{C}) \times_{K_{P}} V \rightarrow \widetilde{E}=P . Z_{F}(\mathbb{C}) \times_{K_{P} . Z_{F}(\mathbb{C})} V .
$$


Let $\bar{E}_{\Sigma}^{\prime}$ denote Mumford's canonical extension of the vector bundle $E^{\prime}=\Gamma \backslash E \rightarrow X$ to the toroidal compactification $\bar{X}_{\Sigma}$, and let $\bar{E}_{F, \Sigma}^{\prime}=\varphi_{F, \Sigma}^{*}\left(E_{\Sigma}^{\prime}\right)$ be its pullback to $D_{F, \Sigma}$. Then we have a further canonical identification $E^{\prime}=\bar{E}_{F, \Sigma}^{\prime}\left|\left(\Gamma_{F}^{\prime} \backslash D\right)=E_{F}^{\prime}\right|\left(\Gamma_{F}^{\prime} \backslash D\right)$. We also have vector bundles $E_{h}=G_{h} \times_{K_{h}} V$ on $F$ and its quotient $E_{Y}^{\prime} \rightarrow Y=\Gamma_{h} \backslash F$.

According to [HZ1] (3.3.9) (which in turn relies on [Har2]), the canonical isomorphism 14.6.1 extends to a vector bundle isomorphism

$$
\psi_{\Sigma}:\left(\bar{E}_{F, \Sigma}^{\prime}, D_{F, \Sigma}\right) \sim\left(E_{F}^{A}, A_{F}\right)
$$

which covers $\pi_{2, \Sigma}$. This is the key point in the argument: the isomorphism $\psi_{\Sigma}$ identifies Mumford's canonical extension (which is defined using a growth condition on a singular connection) with a vector bundle, $\pi_{2, \Sigma}^{*}\left(E_{F}^{A}\right)$ which is defined topologically, and which is trivial on each torus embedding $\pi_{2, \Sigma}^{-1}(a) \cong T_{F, \Sigma}$. We will use this isomorphism to extend the parabolically induced connection over the toroidal compactification, because such a parabolically induced connection is also pulled up from $E_{F}^{A}$.

As in (10.1.1), define an action of $P . Z_{F}(\mathbb{C})$ on the vector bundle $E_{h} \rightarrow F$ by

$$
u g_{h} g_{\ell} z \cdot\left[g_{h}^{\prime}, v\right]=\left[g_{h} g_{h}^{\prime}, \lambda_{1}\left(g_{\ell}\right) v\right]
$$

(where $u \in \mathcal{U}_{P}, g_{h}, g_{h}^{\prime} \in G_{h}, g_{\ell} \in G_{\ell}, z \in Z_{f}(\mathbb{C})$, and $v \in V$ ). Define a mapping

$$
\tilde{\Phi}_{F}: P Z_{F}(\mathbb{C}) \times_{K_{P} Z_{F}(\mathbb{C})} V \rightarrow G_{h} \times_{K_{h}} V
$$

by $\tilde{\Phi}_{F}\left(\left[u g_{h} g_{\ell} z, v\right]\right)=\left[g_{h}, \lambda_{1}\left(g_{\ell}\right) v\right]$. Then $\tilde{\Phi}_{F}$ is well defined, it is $P . Z_{F}(\mathbb{C})$-equivariant, and it gives a $P . Z_{F}(\mathbb{C})$-equivariant isomorphism of vector bundles,

$$
\Phi_{F}:\left(\widetilde{E}, D_{F} / Z_{F}(\mathbb{C})\right) \sim\left(E_{h}, F\right)
$$

which covers $\theta_{1}$. Moreover the composition $\Phi_{F} \Psi \mid(E, D)$ is precisely the isomorphism $\Phi$ : $(E, D) \cong \pi^{*}\left(E_{h}, F\right)$ of $(10.1 .3)$. This array of vector bundles appears in figure 6 .

14.7. Each of the vector bundles in Figure 6 comes equipped with a connection. Let $\nabla_{Y}$ be a given connection on $E_{Y}^{\prime} \rightarrow Y$ and let $\nabla_{h}$ be its pullback to $E_{h} \rightarrow F$. It is $\Gamma_{h}$-invariant. Define $\tilde{\nabla}=\Phi_{F}^{*}\left(\nabla_{h}\right)$ to be the pullback of $\nabla_{h}$ under the isomorphism (14.6.6). By $\S 10.7$ it is $\nu_{h}^{*}\left(\Gamma_{h}\right)$-invariant. Then $\Psi^{*}(\tilde{\nabla})$ is an extension of the parabolically induced connection $\Phi^{*}\left(\nabla_{h}\right)$ on $(E, D)$. Both connections are invariant under $\nu_{h}^{*}\left(\Gamma_{h}\right) \supset \Gamma_{F}^{\prime}$. Let $\nabla_{F}^{\prime}$ denote the resulting connection on the quotient $\left(E_{F}^{\prime}, M_{F}^{\prime}\right.$ ) (where again we use the same symbol to denote this connection as well as its restriction to $\left.\left(E_{F}^{\prime}, \Gamma_{F}^{\prime} \backslash D\right)\right)$. We need to show that this connection $\nabla_{F}^{\prime}$ on $\left(E_{F}^{\prime}, \Gamma_{F}^{\prime} \backslash D\right)$ has a smooth extension to a connection $\nabla_{F, \Sigma}^{\prime}$ on $\left(\bar{E}_{F, \Sigma}^{\prime}, D_{F, \Sigma}\right)$ which is invariant under $\Gamma_{F}^{\prime} \backslash \Gamma_{P}$.

The connection $\tilde{\nabla}$ passes to a connection $\nabla_{F}^{A}$ on $\left(E_{F}^{A}, A_{F}\right)$ such that $\nabla_{F}^{\prime}=\psi^{*}\left(\nabla_{F}^{A}\right)$. Therefore the connection

$$
\nabla_{F, \Sigma}^{\prime}=\psi_{\Sigma}^{*}\left(\nabla_{F}^{A}\right)
$$




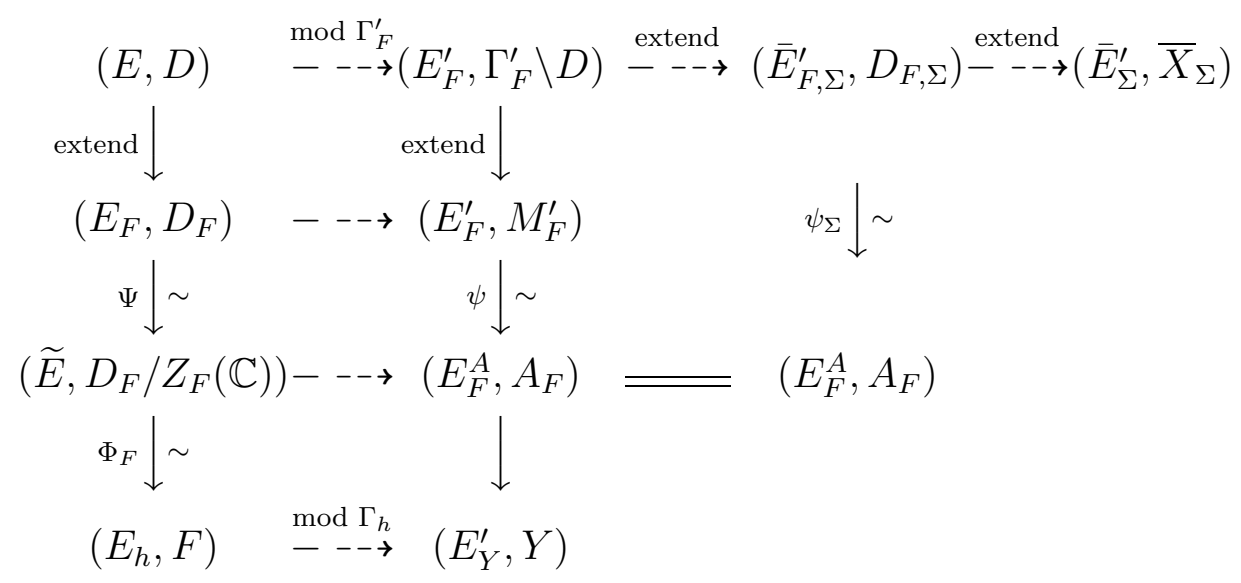

FigURE 6. Vector bundles on the toroidal compactification

on $\left(\bar{E}_{F, \Sigma}^{\prime}, M_{F, \Sigma}^{\prime}\right)$ is a smooth extension of $\nabla_{F}^{\prime}$. The $\Gamma_{F}^{\prime} \backslash \Gamma_{P}$-invariance of $\nabla_{F, \Sigma}^{\prime}$ follows from the $\nu_{h}^{*}\left(\Gamma_{h}\right)$-invariance of $\widetilde{\nabla}$. This completes the proof of Proposition 14.3.

\section{Chern Classes and COnstructible functions}

15.1. A constructible function $F: W \rightarrow \mathbb{Z}$ on a complete (complex) algebraic variety $W$ is one which is constant on the strata of some algebraic (Whitney) stratification of $W$. The Euler characteristic of such a constructible function $F$ is the sum

$$
\chi(W ; F)=\sum_{\alpha} \chi\left(W_{\alpha}\right) F\left(W_{\alpha}\right)
$$

over strata $W_{\alpha} \subset W$ along which the function $F$ is constant. If $f: W \rightarrow W^{\prime}$ is an (proper) algebraic mapping, then the pushforward of the constructible function $F$ is the constructible function

$$
f_{*}(F)\left(w^{\prime}\right)=\chi\left(f^{-1}\left(w^{\prime}\right) ; F\right)
$$

(for any $w^{\prime} \in W^{\prime}$ ). According to [Mac], for each constructible function $F: W \rightarrow \mathbb{Z}$ it is possible to associate a unique Chern class $c_{*}(W ; F) \in H_{*}(W ; \mathbb{Z})$ which depends linearly on $F$, such that $f_{*} c_{*}(W ; F)=c_{*}\left(W^{\prime} ; f_{*} F\right)$ (whenever $f: W \rightarrow W^{\prime}$ is a proper morphism), and such that $c_{*}\left(W ; \mathbf{1}_{W}\right)=c^{*}(W) \cap[W]$ if $W$ is nonsingular. (Here, $[W] \in H_{2 \operatorname{dim}(W)}(W ; \mathbb{Z})$ denotes the fundamental class of $W$.) The MacPherson-Schwartz Chern class of $W$ is the Chern class of the constructible function $\mathbf{1}_{W}$.

15.2. Now let $\bar{Z}$ be a nonsingular complete complex algebraic variety and let $D=D_{1} \cup$ $D_{2} \cup \ldots \cup D_{m}$ be a union of smooth divisors with normal crossings in $\bar{Z}$. Set $Z=\bar{Z}-D$. The tangent bundle $T_{Z}$ of $Z$ has a "logarithmic" extension to $\bar{Z}$,

$$
T_{\bar{Z}}(-\log D)=\operatorname{Hom}\left(\Omega \frac{1}{Z}(\log D), \mathcal{O}_{\bar{Z}}\right)
$$


which is called the "log-tangent bundle" of $(\bar{Z}, D)$. It is the vector bundle whose sheaf of sections near any $\mathrm{k}$-fold multi-intersection $\left\{z_{1}=z_{2}=\ldots=z_{k}=0\right\}$ of the divisors is generated by $z_{1} \frac{\partial}{\partial z_{1}}, z_{2} \frac{\partial}{\partial z_{2}}, \ldots, z_{k} \frac{\partial}{\partial z_{k}}, \frac{\partial}{\partial z_{k+1}}, \ldots, \frac{\partial}{\partial z_{n}}($ where $n=\operatorname{dim}(Z))$. The following result was discovered independently by P. Aluffi [Al].

15.3. Proposition. The Chern class of the log tangent bundle is equal to the Chern class of the constructible function which is 1 on $Z=\bar{Z}-D$, that is,

$$
c^{*}\left(T_{\bar{Z}}(-\log D)\right) \cap[\bar{Z}]=c_{*}\left(\mathbf{1}_{Z}\right) .
$$

15.4. Proof. For any subset $I \subset\{1,2, \ldots, m\}$ let $D_{I}=\bigcap_{i \in I} D_{i}$, let

$$
D^{I}=D_{I} \cap \bigcup_{j \notin I} D_{j}
$$

denote the "trace" of the divisor $D$ in $D_{I}$, and let $D_{I}^{o}=D_{I}-D^{I}$ denote its complement. The restriction of the log tangent bundle of $(\bar{Z}, D)$ to any intersection $D_{I}$ is (topologically) isomorphic to the direct sum of vector bundles

$$
T_{\bar{Z}}(-\log D)\left|D_{I} \cong T_{D_{I}}\left(-\log \left(D^{I}\right)\right) \oplus\right| I \mid \mathbf{1}
$$

(the last symbol denoting $|I|$ copies of the trivial bundle). (This follows from the short exact sequence of locally free sheaves on $D_{j}$,

$$
0 \longrightarrow \Omega_{D_{j}}^{1}\left(\log D^{\{j\}}\right) \longrightarrow \Omega_{\frac{1}{Z}}(\log D) \mid D_{j} \longrightarrow \mathcal{O}_{D_{j}} \longrightarrow 0
$$

by dualizing and induction.) We will prove Proposition 15.3 by induction on the number $m$ of divisors, with the case $m=0$ being trivial. For any constructible function $F$ on $\bar{Z}$, denote by $c(F) \in H^{*}(\bar{Z})$ the Poincaré dual of the (homology) Chern class of $F$. Each divisor $D_{j}$ carries a fundamental homology class whose Poincaré dual we denote by $\left[D_{j}\right] \in H^{2}(\bar{Z})$. The Chern class of the line bundle $\mathcal{O}\left(D_{j}\right)$ is $1+\left[D_{j}\right]$. Let $\tilde{c}$ denote the Chern class of the bundle $T_{\bar{Z}}(-\log D)$. If $I \subset\{1,2, \ldots, m\}$ and if $i: D_{I} \rightarrow \bar{Z}$ denotes the inclusion then

$$
\tilde{c} \cdot\left[D_{I}\right]=i_{*}\left(c\left(T_{\bar{Z}}(-\log D)\right) \mid D_{I}\right)=i_{*} c\left(T_{D_{I}}\left(-\log \left(D^{I}\right)\right)=i_{*} c\left(\mathbf{1}_{D_{I}^{o}}\right)\right.
$$

by (15.4.1) and induction. Using [Tu] Proposition 1.2 we see,

$$
\begin{aligned}
c(\bar{Z}) & =\tilde{c} \cdot \prod_{i}\left(1+\left[D_{i}\right]\right)=\tilde{c}+\tilde{c} \cdot \sum_{I}\left[D_{I}\right] \\
& =\tilde{c}+\sum_{I} c\left(\mathbf{1}_{D_{I}^{o}}\right)=\tilde{c}+c\left(\mathbf{1}_{D}\right)
\end{aligned}
$$

since each point in $D$ occurs in exactly one multi-intersection of divisors. 
15.5. Theorem. Let $X=\Gamma \backslash G / K$ be a Hermitian locally symmetric space as in $\S 9$, with Baily-Borel compactification $\bar{X}$. Let $\bar{c}^{i}(\bar{X}) \in H^{2 i}(\bar{X} ; \mathbb{C})$ denote the cohomology Chern class of the tangent bundle, constructed in Theorem 11.8. Then its homology image

$$
\bar{c}^{*}(\bar{X}) \cap[\bar{X}]=c_{*}\left(\mathbf{1}_{X}\right) \in H_{*}(\bar{X} ; \mathbb{Z})
$$

lies in integral homology and coincides with the (MacPherson) Chern class of the constructible function which is 1 on $X$ and is 0 on $\bar{X}-X$.

15.6. Proof. Let $\tau: \bar{X}_{\Sigma} \rightarrow \bar{X}$ denote a smooth toroidal resolution of singularities, having chosen the system of polyhedral cone decompositions $\Sigma$ so that the exceptional divisor $D$ is a union of smooth divisors with normal crossings. Let $T_{\bar{X}_{\Sigma}}(-\log D)$ denote the $\log$ tangent bundle of $\left(\bar{X}_{\Sigma}, D\right)$. As in [Mu1] Prop. 3.4, this bundle is isomorphic to Mumford's canonical extension $\bar{T}_{X, \Sigma}$ of the tangent bundle. Therefore

$$
\begin{aligned}
\bar{c}^{*}(\bar{X}) \cap[\bar{X}] & \left.=\tau_{*}\left(\tau^{*} \bar{c}^{*}(\bar{X}) \cap\left[\bar{X}_{\Sigma}\right]\right)\right) \\
& =\tau_{*}\left(c^{*}\left(\bar{T}_{X, \Sigma}\right) \cap\left[\bar{X}_{\Sigma}\right]\right) \\
& =\tau_{*} c_{*}\left(\mathbf{1}_{X}\right)=c_{*}\left(\tau_{*}\left(\mathbf{1}_{X}\right)\right)=c_{*}\left(\mathbf{1}_{X}\right)
\end{aligned}
$$

by Theorem 13.2, Proposition 15.3 and (15.1.1).

15.7. Corollary. The MacPherson-Schwartz Chern class of the Baily-Borel compactification $\bar{X}$ is given by the sum over strata $Y \subset \bar{X}$,

$$
c_{*}\left(\mathbf{1}_{\bar{X}}\right)=c_{*}\left(\sum_{Y \subset \bar{X}} \mathbf{1}_{Y}\right)=\sum_{Y \subset \bar{X}} i_{*} \bar{c}^{*}(\bar{Y}) \cap[\bar{Y}]
$$

where $i: \bar{Y} \hookrightarrow \bar{X}$ is the inclusion of the closure of $Y$ (which is also the Baily-Borel compactification of $Y$ ) into $\bar{X}$.

\section{Cohomology of the Baily-Borel Compactification}

16.1. Let $K$ be a compact Lie group and let $E K \rightarrow B K$ be the universal principal $K$-bundle. For any representation $\lambda: K \rightarrow G L(V)$ on a complex vector space $V$, let $E_{\lambda}=E K \times{ }_{K} V$ be the associated vector bundle. The Chern classes $c^{i}(E) \in H^{2 i}(B K ; \mathbb{C})$ of all such vector bundles generate a subalgebra which we denote $H_{\text {Chern }}^{*}(B K ; \mathbb{C})$. Two cases are of particular interest: if $K=\mathbf{U}(\mathbf{n})$ then $B K=\lim _{k \rightarrow \infty} G_{n}\left(\mathbb{C}^{n+k}\right)$ is the infinite Grassmann manifold and $H^{*}(B K ; \mathbb{C})=H_{\text {Chern }}^{*}(B K ; \mathbb{C})$. In fact, the standard representation $\lambda: \mathbf{U}(\mathbf{n}) \rightarrow \mathbf{G L}_{\mathbf{n}}(\mathbb{C})$ gives rise to a single vector bundle $E_{\lambda} \rightarrow B K$ such that the algebra $H^{*}(B K ; \mathbb{C})$ is canonically isomorphic to the polynomial algebra in the Chern classes $c^{1}\left(E_{\lambda}\right), c^{2}\left(E_{\lambda}\right), \ldots, c^{n}\left(E_{\lambda}\right)$. If $K=\mathbf{S O}(\mathbf{n})$ then $B K=\lim _{k \rightarrow \infty} G_{n}^{o}\left(\mathbb{R}^{n+k}\right)$ is the infinite Grassmann manifold of real oriented n-planes. Let $\tilde{\lambda}: \mathbf{S O}(\mathbf{n}) \rightarrow \mathbf{G L}_{\mathbf{n}}(\mathbb{R})$ be the standard representation with resulting vector bundle $E_{\tilde{\lambda}} \rightarrow B K$, and let $\lambda: \mathbf{S O}(\mathbf{n}) \rightarrow \mathbf{G L}_{\mathbf{n}}(\mathbb{C})$ 
denote the composition of $\tilde{\lambda}$ with the inclusion $\mathbf{G L}_{\mathbf{n}}(\mathbb{R}) \subset \mathbf{G L}_{\mathbf{n}}(\mathbb{C})$. The associated vector bundle $E_{\lambda}=E_{\tilde{\lambda}}(\mathbb{C})$ is the complexification of $E_{\tilde{\lambda}}$. If $n$ is odd, then $H^{*}(B K ; \mathbb{C})$ is canonically isomorphic to the polynomial algebra generated by the Pontrjagin classes $p^{i}\left(E_{\tilde{\lambda}}\right)=c^{2 i}\left(E_{\lambda}\right) \in H^{4 i}(B K ; \mathbb{C})$ for $i=1,2, \ldots, n$. Hence $H^{*}(B K ; \mathbb{C})=H_{\text {Chern }}^{*}(B K ; \mathbb{C})$. If $n$ is even then the algebra $H^{*}(B K ; \mathbb{C})$ has an additional generator, the Euler class $e=e\left(E_{\tilde{\lambda}}\right) \in H^{n}(B K ; \mathbb{C})$. (It satisfies $e^{2}=p^{n / 2}$.) If $n=2$ then $e$ is the first Chern class of the line bundle corresponding to the representation $\mathbf{S O}(2) \cong \mathbf{U}(1) \subset \mathbf{G L}_{\mathbf{1}}(\mathbb{C})$.

16.2. Now suppose that $K=K_{1} \times K_{2} \times \ldots \times K_{r}$ is a product of unitary groups, odd orthogonal groups, and copies of $\mathbf{S O}(\mathbf{2})$. According to the preceding paragraph, there are representations $\lambda_{1}, \ldots, \lambda_{r}$ of $K$ on certain complex vector spaces $V_{1}, V_{2}, \ldots, V_{r}$ so that the Chern classes of the resulting "universal" complex vector bundles $E_{i}=E K \times_{K} V_{i} \rightarrow B K$ generate the polynomial algebra $H^{*}(B K ; \mathbb{C})=H_{\text {Chern }}^{*}(B K ; \mathbb{C})$.

16.3. Suppose that $\mathbf{G}$ is a semisimple algebraic group defined over $\mathbb{Q}$, and that $\mathbf{G}(\mathbb{R})^{0}$ acts as the identity component of the group of automorphisms of a Hermitian symmetric space $D=G / K$. Recall $([\mathrm{He}] \mathrm{X} \S 6,[\mathrm{Bo} 2])$ that the irreducible components of $D$ come from the following list:

\begin{tabular}{|l|l|l|}
\hline Type & Symmetric Space & Compact Dual \\
\hline AIII & $\mathbf{U}(\mathbf{p}, \mathbf{q}) / \mathbf{U}(\mathbf{p}) \times \mathbf{U}(\mathbf{q})$ & $\mathbf{U}(\mathbf{p}+\mathbf{q}) / \mathbf{U}(\mathbf{p}) \times \mathbf{U}(\mathbf{q})$ \\
DIII & $\mathbf{S O}^{*}(\mathbf{2 n}) / \mathbf{U}(\mathbf{n})$ & $\mathbf{S O}(\mathbf{2 n}) / \mathbf{U}(\mathbf{n})$ \\
$\mathrm{BDI}$ & $\mathbf{S O}(\mathbf{p}, \mathbf{2}) / \mathbf{S O}(\mathbf{p}) \times \mathbf{S O}(\mathbf{2})$ & $\mathbf{S O}(\mathbf{p}+\mathbf{2}) / \mathbf{S O}(\mathbf{p}) \times \mathbf{S O}(\mathbf{2})$ \\
$\mathrm{CI}$ & $\mathbf{S p}(\mathbf{n}, \mathbb{R}) / \mathbf{U}(\mathbf{n})$ & $\mathbf{S p}(\mathbf{n}) / \mathbf{U}(\mathbf{n})$ \\
EIII & $\mathbf{E}_{\mathbf{6}}^{\mathbf{3}} / \mathbf{S p i n}(\mathbf{1 0}) \times \mathbf{S O}(\mathbf{2})$ & $\mathbf{E}_{\mathbf{6}} / \operatorname{Spin}(\mathbf{1 0}) \times \mathbf{S O}(\mathbf{2})$ \\
EVII & $\mathbf{E}_{\mathbf{7}}^{\mathbf{3}} / \mathbf{E}_{\mathbf{6}} \times \mathbf{S O}(\mathbf{2})$ & $\mathbf{E}_{\mathbf{7}} / \mathbf{E}_{\mathbf{6}} \times \mathbf{S O}(\mathbf{2})$ \\
\hline
\end{tabular}

Let $X=\Gamma \backslash G / K$, with $\Gamma \subset \mathbf{G}(\mathbb{Q})$ a neat arithmetic group, and let $\bar{X}$ denote the BailyBorel compactification of $X$. Let $\check{D}=G_{u} / K$ be the compact dual symmetric space, where $G_{u} \subset \mathbf{G}(\mathbb{C})$ is a compact real form containing $K$. The principal bundles $\Gamma \backslash G \rightarrow X$ and $G_{u} \rightarrow \check{D}$ are classified by mappings $\Phi: X \rightarrow B K$ and $\Psi: \check{D} \rightarrow B K$ (respectively) which are uniquely determined up to homotopy. A theorem of Borel [Bo2] states that (in this Hermitian case) the resulting homomorphism $\Psi^{*}: H^{*}(B K ; \mathbb{C}) \rightarrow H^{*}(\check{D} ; \mathbb{C})$ is surjective.

Suppose the irreducible factors of $D=G / K$ are of type AIII, DIII, CI, or BDI for $p$ odd or $p=2$. The construction of controlled Chern forms in Section 11 determines a homomorphism $\widetilde{\Phi}^{*}: H^{*}(B K ; \mathbb{C}) \rightarrow H^{*}(\bar{X} ; \mathbb{C})$ by setting $\widetilde{\Phi}^{*}\left(c^{i}\left(E_{j}\right)\right)=\bar{c}^{i}\left(E_{j}^{\prime}\right)$ (where $E_{j} \rightarrow B K$ is the universal vector bundle corresponding to the representation $\lambda_{j}$ of $\S 16.2$ and $E_{j}^{\prime} \rightarrow X$ is the corresponding automorphic vector bundle). Let us denote the image of $\widetilde{\Phi}^{*}$ by $H_{\text {Chern }}^{*}(\bar{X} ; \mathbb{C})$.

16.4. Theorem. Suppose $X=\Gamma \backslash G / K$ is a Hermitian locally symmetric space such that the irreducible factors of $D=G / K$ are of type AIII, DIII, CI, or BDI for $p$ odd or $p=2$. Then 
the mappings $\widetilde{\Phi}^{*}$ and $\Psi^{*}$ determine a surjection

$$
h: H_{\text {Chern }}^{*}(\bar{X} ; \mathbb{C}) \rightarrow H^{*}(\check{D} ; \mathbb{C})
$$

from this subalgebra of the cohomology of the Baily-Borel compactification, to the cohomology of the compact dual symmetric space. Moreover, for each "universal" vector bundle $E_{j} \rightarrow$ $B K$ we have

$$
h\left(\bar{c}^{i}\left(E_{j}^{\prime}\right)\right)=c^{i}\left(\check{E}_{j}\right)
$$

where $E_{j}^{\prime} \rightarrow X$ and $\check{E}_{j} \rightarrow \check{D}$ are the associated automorphic and homogeneous vector bundles, respectively.

16.5. Proof. Define the mapping $h: H_{\text {Chern }}^{*}(\bar{X} ; \mathbb{C}) \rightarrow H^{*}(\check{D} ; \mathbb{C})$ by $h \tilde{\Phi}^{*}(c)=\Psi^{*}(c)$ for any $c \in H^{*}(B K ; \mathbb{C})$. If this is well defined, it is surjective by Borel's theorem. To show it is well defined, let us suppose that $\tilde{\Phi}^{*}(c)=0$. We must show that $\Psi^{*}(c)=0$, so we assume the contrary.

Let $x=\Psi^{*}(c) \in H^{i}(\check{D} ; \mathbb{C})$. By Poincaré duality, there exists a complementary class $y \in H^{2 n-i}(\check{D} ; \mathbb{C})$ so that $(x \cup y) \cap[\check{D}] \neq 0$ (where $n=\operatorname{dim}_{\mathbb{C}}(\check{D})$ ). Then $y$ has a lift, $d \in H^{2 n-i}(B K ; \mathbb{C})$ with $\Psi^{*}(d)=y$. Let us write $K=K_{1} K_{2} \ldots K_{r}$ for the decomposition of $K$ into irreducible factors. By $\S 16.2$ the polynomial algebra $H^{*}(B K ; \mathbb{C})$ is generated by the Chern classes of the universal vector bundles $E_{1}, E_{2}, \ldots, E_{r}$ corresponding to representations $\lambda_{i}: K_{i} \rightarrow G L\left(V_{i}\right)$. Hence, both $c$ and $d$ are polynomials in the Chern classes of the vector bundles $E_{1}, E_{2}, \ldots, E_{r}$. Hence $\left(\widetilde{\Phi}^{*}(c) \cup \widetilde{\Phi}^{*}(d)\right) \cap[\bar{X}] \in \mathbb{C}$ is a sum of "generalized" Chern numbers which, by the Proposition 13.4, coincides with the corresponding sum of "generalized" Chern numbers for the compact dual symmetric space, $v(\Gamma)(x \cup y) \cap[\check{D}] \neq 0$. This implies that $\tilde{\Phi}^{*}(c) \neq 0$ which is a contradiction.

16.6. Remarks. We do not know whether the surjection (16.4.1) has a canonical splitting. However, the intersection cohomology $I H^{*}(\bar{X} ; \mathbb{C})$ contains, in a canonical way, a copy of the cohomology $H^{*}(\check{D} ; \mathbb{C})$ of the compact dual symmetric space. By the Zucker conjecture $([\mathrm{Lo}]$ and $[\mathrm{SS}]$ ), the intersection cohomology may be identified with the $L^{2}$ cohomology of $X$ which, in turn may be identified with the relative Lie algebra cohomology $H^{*}\left(\mathfrak{g}, K ; L^{2}(\Gamma \backslash G)\right)$. But $L^{2}(\Gamma \backslash G)$ contains a copy of the trivial representation $\mathbf{1}$ (the constant functions), whose cohomology $H^{*}(\mathfrak{g}, K ; \mathbf{1}) \cong H^{*}(\check{D} ; \mathbb{C})$ is the cohomology of the compact dual symmetric space. We sketch a proof that the following diagram commutes.

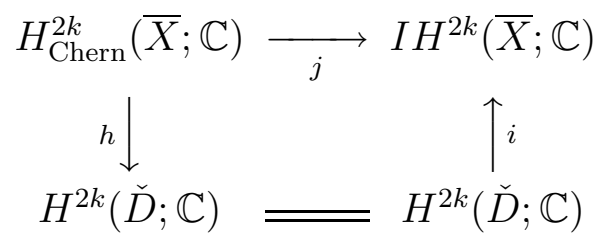

If $E^{\prime} \rightarrow X$ and $\check{E} \rightarrow \check{D}$ are vector bundles arising from the same representation $\lambda$ of $K$ then the class $j\left(\bar{c}^{k}\left(E^{\prime}\right)\right)$ is represented by the differential form $\sigma^{k}\left(\nabla_{X}^{\mathrm{p}}\right)$ which is controlled, 
hence bounded, hence $L^{2}$. The class $i\left(c^{k}(\check{E})\right)$ is represented by the differential form $\sigma^{k}\left(\nabla_{X}^{\text {Nom }}\right)$ which is "invariant" (meaning that its pullback to $D$ is invariant), hence $L^{2}$. The intersection cohomology of $\bar{X}$ embeds into the ordinary cohomology of any toroidal resolution $\bar{X}_{\Sigma}$. But when these two differential forms are considered on $\bar{X}_{\Sigma}$, they both represent the same cohomology class, $c^{k}\left(\bar{E}_{\Sigma}^{\prime}\right)$ (using Theorem 13.2 and [Mu1]). Alternatively, one may deform the connection $\nabla_{X}^{\mathrm{p}}$ to $\nabla_{X}^{\mathrm{Nom}}$, obtaining a differential form $\Psi \in \mathcal{A}^{2 k-1}(X)$ such that $d \Psi=$ $\sigma^{k}\left(\nabla_{X}^{\mathrm{p}}\right)-\sigma^{k}\left(\nabla_{X}^{\mathrm{Nom}}\right)$, and check that $\Psi$ is $L^{2}$.

In the case BDI, the compact dual is $\check{D}=S O(p+2) / S O(p) \times S O(2)$. Suppose $p$ is even.

Its cohomology $H^{*}(\check{D} ; \mathbb{C})$ has a basis $\left\{1, c_{1}, c_{1}^{2}, \ldots, c_{1}^{p-1}, e\right\}$ where $c_{1}$ is the Chern class of the complexification of the line bundle arising from the standard representation of $S O(2)$ and where $e$ is the Euler class of the vector bundle arising from the standard representation of $S O(p),[\mathrm{BoH}] \S 16.5$. All these classes lift canonically to $I H^{*}(\bar{X} ; \mathbb{C})$ and $c_{1}^{j}$ lifts further to the (ordinary) cohomology of the Baily-Borel compactification. However (except in the case $p=2)$ we do not know whether $e$ also lifts further to $H^{*}(\bar{X} ; \mathbb{C})$.

\section{REFERENCES}

[Ak] E. Akin, Stiefel Whitney homology classes and bordism. Trans. Amer. Math. Soc. 205 (1975), pp. 341-359.

[Al] P. Aluffi, Differential forms with logarithmic poles and the Chern-Schwartz-MacPherson classes of singular varieties, C.R. Acad. Sci. Paris, Sér I. Math. 329 (1999), pp. 619-624.

[AMRT] A. Ash, D. Mumford, M. Rapoport, Y.-S. Tai, Smooth Compactification of Locally Symmetric Varieties Math. Sci. Press, Brookline MA, 1975.

[ADS] M. F. Atiyah, H. Donnelly, and I. M. Singer, Eta invariants, signature defects of cusps, and values of $L$-functions. Ann. of Math 118 (1983), 131-177.

[BB] W. Baily and A. Borel, Compactification of arithmetic quotients of bounded symmetric domains, Ann. of Math. 84 (1966), pp. 442-528.

[BBF] G. Barthel, J.-P. Brasselet, K.-H. Fieseler, O. Gabber, and L. Kaup, Relèvement de cycles algébriques et homomorphismes associés en homologie d'intersection, Ann. of Math. 141 (1995), pp. 147-179.

[BFM] P. Baum, W. Fulton, and R. MacPherson, Riemann-Roch for singular spaces, Publ. Math. I.H.E.S. 45 (1975), pp. 101-145.

[BGV] N. Berline, E. Getzler, and M. Vergne, Heat Kernels and Dirac Operators, Grundlehren 298, Springer Verlag, New York, 1992.

[Bo2] A. Borel, Sur la cohomologie des espaces fibrés principaux et des espaces homogènes de groupes de Lie compacts, Ann. of Math. 57 (1953), pp. 115-207.

[Bo3] A. Borel, Linear Algebraic Groups (second edition), Graduate Texts in Mathematics 126, Springer Verlag, N. Y., 1991.

$[\mathrm{BoH}] \quad$ A. Borel and F. Hirzebruch, Characteristic classes and homogeneous spaces I, Amer. J. Math. 80 (1958), pp. 458-538.

[BoS] A. Borel and J.P. Serre, Corners and arithmetic groups, Comment. Math. Helv. 48 (1973), pp. 436-491.

[BW] J.-P. Brasselet and A. Weber, A canonical lift of Chern-Mather classes to intersection homology, preprint, Luminy 1997. 
[CL] R. Charney and R. Lee, Cohomology of the Satake compactification, Topology 22 (1983), pp. 389-423.

[D] P. Deligne, Équations différentielles à points singuliers réguliers Lecture Notes in Mathematics 52, Springer Verlag, New York, 1970.

[FC] G. Faltings and C.-L. Chai, Degeneration of Abelian Varieties, Ergeb. Math. 222, Springer Verlag, N.Y., 1990.

[Gi] C. Gibson, K. Wirthmüller, A. du Plessis, and E. Looijenga, Topological Stability of Smooth Mappings Lecture Notes in Mathematics 552, Springer Verlag, N.Y., 1976

[Go1] M. Goresky, Whitney stratified chains and cochains, Trans. Amer. Math. Soc. 267 (1981), pp. 175-196.

[Go2] M. Goresky, Intersection homology operations, Comm. Math. Helv. 59 (1984), pp. $485-505$.

[GHM] M. Goresky, G. Harder, and R. MacPherson, Weighted cohomology. Invent. Math. 116 (1994), pp. $139-213$.

[GM1] M. Goresky and R. MacPherson, Intersection homology theory, Topology 19 (1980), pp. 135-162.

[GM2] M. Goresky and R. MacPherson, Intersection homology II, Invent. Math. 71 (1983), pp. 77-129.

[GM3] M. Goresky and R. MacPherson, Stratified Morse Theory, Ergeb. Math. 14, Springer Verlag, Berlin, 1988.

[GM4] M. Goresky and R. MacPherson, The topological trace formula. Preprint, Inst. for Adv. Study, May 2001.

[GP] M. Goresky and W. Pardon, Wu numbers of singular spaces, Topology 28 (1989), pp. 325-367.

[GT] M. Goresky and Y.-S. Tai, Toroidal and reductive Borel-Serre compactifications of locally symmetric spaces. Amer. J. Math. 121 (1999), pp. 1095-1151.

[Gr1] P. Griffiths, Periods of integrals on algebraic manifolds: summary of main results and discussion of open problems, Bull. Amer. Math. Soc. 76 (1970), pp. 228-296.

[Gr2] P. Griffiths, Periods of integrals on algebraic manifolds III, Publ. Math. I.H.E.S. 38 (1970), pp. $125-180$.

[H-T] S. Halperin and D. Toledo, Stiefel-Whitney homology classes, Ann. of Math. 96 (1972), pp. $511-525$.

[Ha1] R. Hardt, Topological properties of subanalytic sets. Trans. Amer. Math. Soc. 211 (1975), pp. 57-70.

[Ha2] R. Hardt, Stratifications of real analytic mappings and images. Invent. Math. 28 (1975), pp. 193208.

[Har1] M. Harris, Arithmetic vector bundles and automorphic forms on Shimura varieties I, Invent. Math. 82 (1985), pp. 151-189.

[Har2] M. Harris, Arithmetic vector bundles and automorphic forms on Shimura varieties II, Comp. Math. 60 (1986), pp. 323-378.

[Har3] M. Harris, Functorial properties of toroidal compactifications of locally symmetric varieties, Proc. Lon. Math. Soc. 59 (1989), pp. 1-22.

[Har4] M. Harris, Automorphic forms of $\bar{\partial}$-cohomology type as coherent cohomology classes, J. Diff. Geom. 32 (1990), pp. 1-63.

[HZ1] M. Harris and S. Zucker, Boundary cohomology of Shimura varieties I, Ann. Sci. École Norm. Sup. 27 (1994), pp. 249-344.

[HZ2] M. Harris and S. Zucker, Boundary cohomology of Shimura varieties II. Hodge theory at the boundary, Invent. Math. 116 (1994), pp. 243-308.

[He] S. Helgason, Differential Geometry, Lie Groups, and Symmetric Spaces, Academic Press, 1978. 
[Hi1] H. Hironaka, Subanalytic sets, in Number Theory, Algebraic Geometry and Commutative Algebra, volume in honor of A. Akizuki, Kinokunya Tokyo 1973, pp. 453-493.

[Hi2] H. Hironaka, Stratification and flatness, in Real and Complex Singularities, Nordic Summer School (Oslo, 1976). Sijthoff-Noordhoff, Groningen 1977.

[Hr1] F. Hirzebruch, Automorphe Formen und der Satz von Riemann-Roch, in: Symposium Internacional de Topologia Algebraica, Unesco, 1958.

[Hr2] F. Hirzebruch, Hilbert modular surfaces, Enseign. Math. 19 (1973), pp. 183-281.

[Hr3] F. Hirzebruch, letter to M. F. Atiyah, Kommentare $§ 38$ Collected Works, Volume II, pp. 773-774, Springer Verlag, Heidelberg, 1987.

[KN] S. Kobayashi and K. Nomizu, Foundations of Differential Geometry I,II, John Wiley and Sons, N.Y., 1966.

[Lo] E. Looijenga, $L^{2}$ cohomology of locally symmetric varieties. Comp. Math. 67 (1988), pp. 3-20.

[Mac] R. MacPherson, Chern classes for singular varieties, Ann. of Math. 100 (1974), pp. 423-432.

[Mat] J. Mather, Notes on Topological Stability, mimeographed notes, Harvard University, 1970.

[Mi] J. Milnor, On the cobordism ring $\Omega^{*}$ and a complex analogue. Amer. J. Math. 82 (1960), pp. 505-521.

[MiS] J. Milnor and J. Stasheff, Characteristic Classes, Princeton University Press, Princeton N.J., 1974 .

[Mü] W. Müller, Signature defect of cusps of Hilbert modular varieties and values of $L$-series at $s=1$, J. Diff. Geom. 20 (1984), pp. 55-119.

[Mu1] D. Mumford, Hirzebruch's proportionality theorem in the non-compact case, Invent. Math. 42 (1977), pp. 239-272.

[Na] Y. Namikawa, Toroidal Compactification of Siegel Spaces, Lecture Notes in Mathematics 812, Springer Verlag, N.Y., 1980.

[No] K. Nomizu, Invariant affine connections on homogeneous spaces, Amer. J. Math 76 (1954), 33-65.

[P] W. Pardon, Intersection homology Poincaré spaces and the characteristic variety theorem, Comment. Math. Helv. 65 (1990), pp. 198-233.

[Sa1] L. Saper, Tilings and finite energy retractions of locally symmetric spaces. Comment. Math. Helv. 72 (1997), pp. 167-202.

[SS] L. Saper and M. Stern, $L_{2}$ cohomology of arithmetic varieties, Ann. of Math. 132 (1990), pp. 1-69.

[Sat] I. Satake, Algebraic Structures of Symmetric Domains, Princeton University Press, Princeton N.J., 1980.

[Sp] T. A. Springer, Conjugacy classes in algebraic groups, Group Theory, Beijing 1984, Springer Lecture Notes in Mathematics 1185, pp. 175-209.

[St] R. Stong, Notes on Cobordism Theory. Princeton University Press, Princeton N.J., 1958.

[St1] M. Stern, $L^{2}$ index theorems on locally symmetric spaces, Invent. Math. 96 (1989), pp. 231-282.

[Su] D. Sullivan, Combinatorial invariants of analytic spaces. Proceedings of Liverpool Singularities Symposium I, Springer Lecture Notes in Mathematics 192, Springer Verlag, N.Y., 1970.

[T] B. Totaro, Chern numbers for singular varieties and elliptic cohomology, preprint, Univ. of Chicago, 1997.

[Tu] R. Tsushima, A formula for the dimension of spaces of Siegel cusp forms of degree three. Amer. J. Math. 102 (1980), pp. 937-977.

[V] A. Verona, Le théorème de de Rham pour les préstratifications abstraits, C. R. Acad Sci. Paris Sér A 273 (1971), pp. A886-A889.

[Wa] H. C. Wang, On invariant connections over a principal fibre bundle, Nagoya Math J., 13 (1958), $1-19$. 
[WK] J. Wolf and A. Korányi, Generalized Cayley transformations of bounded symmetric domains, Amer. J. of Math. 87 (1965), pp. 899-939.

[Z1] S. Zucker, $L_{2}$ cohomology of warped products and arithmetic groups. Invent. Math. 70 (1982), pp. 169-218.

[Z2] S. Zucker, Satake compactifications, Comment. Math. Helv. 58 (1983), pp. 312-343.

[Z3] S. Zucker, On the boundary cohomology of locally symmetric spaces, Vietnam J. Math., 25 (1997), pp. 279-318. 